\title{
Site-specific gains and losses of heterochromatin accelerate the age-related neurodegeneration through the cascading destruction of KDM3B- centered epigenomic network
}

\author{
Mi-Jin An \\ Ji-Young Kim \\ Jinhong Park
}

Chung-Ang University

Chung-Ang University

Jinho Kim

Chung-Ang University

Dae-Hyun Kim

Chung-Ang University

Geun-Seup Shin

Chung-Ang University

Hyun-Min Lee

Chung-Ang University

Ah-Ra Jo

Chung-Ang University

Chul-Hong Kim

Chung-Ang University

Mi Jin Kim

Chung-Ang University

Jeongkyu Kim

Chung-Ang University

Sangmyung Rhee

Chung-Ang University

\section{Sang-Beom Seo}

Chung-Ang University

Jung-Woong Kim ( $\square$ jungkim@cau.ac.kr)

Chung-Ang University https://orcid.org/0000-0003-4458-7213 
Keywords: heterochromatin, aging, Kdm3b, epigenome, network, retina, homeostasis, cone photoreceptor, synapse, apoptosis

Posted Date: January 14th, 2022

DOI: https://doi.org/10.21203/rs.3.rs-1246914/v1

License: (1) This work is licensed under a Creative Commons Attribution 4.0 International License. Read Full License 
1 Site-specific gains and losses of heterochromatin accelerate the age-related neurodegeneration through the cascading destruction of KDM3B-centered epigenomic network

5 Mi-Jin An ${ }^{1,2}$, Ji-Young Kim ${ }^{1,2}$, Jinhong Park ${ }^{1}$, Jinho Kim ${ }^{1}$, Dae-Hyun Kim ${ }^{1}$, Geun-Seup Shin ${ }^{1}$, Hyun-Min Lee $^{1}$, Ah-Ra Jo ${ }^{1}$, Chul-Hong Kim ${ }^{1}$, Mi Jin Kim ${ }^{1}$, Jeongkyu Kim ${ }^{1}$, Sangmyung Rhee ${ }^{1}$, Sang-Beom Seo ${ }^{1}$ and Jung-Woong $\mathrm{Kim}^{1, *}$

8

Keywords:

heterochromatin, aging, $\mathrm{Kdm} 3 \mathrm{~b}$, epigenome, network, retina, homeostasis, cone photoreceptor, synapse, apoptosis

\section{"Corresponding author:}

Jung-Woong Kim, Ph.D., Professor

Department of Life Science, Chung-Ang University, Seoul 06974, South Korea

Tel: +82-2-820-6682, Fax: +82-2-815-6682

Email: jungkim@cau.ac.kr 
ABSTRACT

Epigenetic alterations explained by the "loss of heterochromatin" model have been proposed as a universal mechanism of aging, but the region-specific changes of heterochromatin during aging are unclear. Here, we examine age-dependent transcriptomic profiling of mouse retinal neurons to identify epigenetic regulators involved in heterochromatin loss. RNA sequencing analysis revealed gradual down-regulation of $K d m 3 b$ during retinal aging. Disruption of $K d m 3 b\left(K d m 3 b^{+/}\right)$in 12-month-old mouse retina decreased the number of cone photoreceptors and changed the morphology of cone ribbon synapses. Integration of transcriptome profiling with epigenomic analysis demonstrated gain of heterochromatin feature in synapse assembly and vesicle transport genes via the accumulation of H3K9 mono- and di-methylation. However, the loss of heterochromatin in apoptotic genes exacerbated retinal neurodegeneration. We propose that this KDM3Bcentered epigenomic network is crucial for maintaining cone photoreceptor homeostasis via the modulation of gene-set specific heterochromatin features during aging. 


\section{INTRODUCTION}

Aging affects all organ systems, but age-related nervous system deficits are among the most prominent aging-related changes. Neuronal functions are gradually impaired during aging via aberrant neuronal network activity, increased oxidative stress, and disturbed energy homeostasis ${ }^{1}$. The retina exhibits elaborate cellular patterns and circuitry and is one of the best-understood models of the vertebrate central nervous system; the differentiated retina, a functional aging model, is suitable for demonstrating the destruction between physical neuronal networks (synapses) and molecular regulatory ${ }^{2}$. Retinal aging leads to a reduction in visual acuity and visual field sensitivity, and an increased dark adaptation threshold, resulting in age-related diseases, such as age-related macular degeneration (AMD). Although these physiological changes are apparent, the molecular mechanisms underlying neurochemical changes and genome-wide epigenetic homeostasis during aging have not been identified.

Epigenetic regulation directly contributes to aging and age-related diseases, including changes in chromatin accessibility, accumulation of histone variants, aberrant histone modifications, and chromatin organization ${ }^{3}$. The "loss of heterochromatin" is considered a common mechanism of aging across cell types and species. Age-related destabilization of tightly compacted chromatin leads to aberrant gene expression and cellular dysfunction. A typical mark of constitutive heterochromatin is the trimethylation of histone $\mathrm{H} 3$ on lysine 9 (H3K9me3), while $\mathrm{H} 3 \mathrm{~K} 27 \mathrm{me} 3$ is usually enriched in facultative heterochromatin ${ }^{4}$. Agingassociated losses of repressive histone mark occur at H3K9me3 and H3K27me3. For example, histone methyl transferase SUV39H1 expression levels in hematopoietic stem cells decrease during aging, resulting in a global reduction in $\mathrm{H} 3 \mathrm{~K} 9 \mathrm{me} 3$ level and perturbed heterochromatin function ${ }^{5}$. In addition, $\mathrm{EZH} 2$ is downregulated in stressed and senescent human lung cells, leading H3K27me3 loss ${ }^{6}$. However, the regionspecific changes in heterochromatin of the functionally active genes in differentiated cells have not been elucidated.

The epigenomic network is controlled by chromatin modification and the binding of transcription factors, resulting in changes in gene expression. The resulting variability in the pattern of epigenetic 
information within individual cells in a population during aging leads to transcriptional drifts and genomic instability. Histone-modifying enzymes are regulatory factors for gene expression; among them, histone lysine methyl transferases and demethylases (KDMs) establish functional cell types through dynamic writing and erasing methylation marks on cell-type specific gene elements during development and aging 7,8 . The lysine-specific demethylase 3 (KDM3) family includes KDM3A, KDM3B, and KDM3C, which are specific demethylases for mono-, and di-methylation at $\mathrm{H} 3 \mathrm{~K} 9$ and act as transcriptional activators ${ }^{9}$. The KDM3 family is crucial for cell survival and proliferation in rapidly dividing cells, such as prostate cancer, leukemia, and hepatocellular carcinoma cells ${ }^{9-11}$. Nevertheless, whether and how KDM3 contributes to nonmitotic differentiated cells in development, aging, and disease development is still poorly understood.

The functional physiology of differentiated cells is manifested through genetically encoded molecular interactions and complex networks. Multi-dimensional epigenomic networks can shed light on gene regulatory networks (GRNs) in in vivo systems, resulting in the visualization of hidden biomolecular interactions. GRNs reveal cell-type specific development, differentiation, and cellular functions that control the transcriptional expression of signal molecules and transcription factors, and regulatory interactions ${ }^{12,13}$. The network structure of integrated genomes and epigenomes contributes to better understanding of spatial and temporal regulation of gene expression during development and aging ${ }^{14}$. It is also possible to measure the network strength of gene regulation-epigenetic modification via the perpetual imbalance of homeostasis during aging.

Herein, we aimed to understand these epigenomic networks using genetic approaches, integrated with physiological assays and multiple genome-wide analyses using fully differentiated retina as an aging model system. We found that haploinsufficiency of $K d m 3 b$ is associated with a decreased number of cone photoreceptors via increased apoptosis and induced alterations of cone ribbon synapses due to the sitespecific alteration of heterochromatin features. Furthermore, KDM3B is required for the maintenance of the integrated GRN that confers normal homeotic regulation in the mouse retina during aging. 


\section{RESULTS}

\section{Characterization of heterochromatin organization and transcriptomic profile involved in retinal} aging. The retina is composed of six neuronal cell types, including rod and cone photoreceptors (Fig. 1a). To investigate the organization pattern of chromatin during retinal aging, we observed the distribution of heterochromatin in the nuclei of photoreceptors using DAPI staining. Heterochromatin was detected at the nuclear periphery and within chromocenters in cone photoreceptors of 2-month-old mice, but less condensed heterochromatin was observed in the center of the nucleus in 18-month-old mice (Fig. 1b). However, the distribution of heterochromatin did not change in the rods of both 2- and 18-month-old mice. The number and mean area of chromocenters in cone nuclei decreased in the 18-month-old mice compared to that in the 2-month-old mice (Fig. 1c and Extended Data Fig. 1a). Several studies have shown that repressive histone modifications, such as $\mathrm{H} 3 \mathrm{~K} 9 \mathrm{me} 2 / 3$, are hallmarks of pericentromeric heterochromatin that play a pivotal role in heterochromatin formation ${ }^{15,16}$. We also observed the distribution of silencing histone modification (H3K9me1/2/3) and active modification (H3K9ac and H3K4me3) in cones and rods during aging. $\mathrm{H} 3 \mathrm{~K} 9 \mathrm{me} 2 / 3$ was abundant in the heterochromatin at the nuclear periphery in 2-month-old mice with conventional nuclear architecture, whereas H3K9me2/3 showed unusual distribution in both euchromatin and heterochromatin (but not in chromocenters) in 18-month-old mice (Extended Data Fig. 1b). We did not observe any differences in the distributions of $\mathrm{H} 3 \mathrm{~K} 9 \mathrm{me} 1$ and active modification between 2-month-old mice and 18-month-old mice (Extended Data Fig. 1b). Furthermore, we confirmed the global histone modification of whole retina using immunoblotting (Extended Data Fig. 1c). H3K9me2 level was slightly decreased in 18-month-old mice, whereas H3K9me3 level was increased in whole retina of 18month-old mice. These data indicate that genome-wide organization of chromatin is altered during retina aging.

To investigate whether epigenetic regulators are involved in "loss of heterochromatin features" during neuronal aging, we performed gene expression profiling using RNA-sequencing analysis of 1-, 6-, 
12-, and 18-month-old mice retina. Strong agreement was observed between the results of each biological replicate from the different time points (Extended Data Fig. 2a). Given the criteria of FPKM $>30.0$ and coefficient of variation between each biological replicate within 20\%, 1,615 differentially expressed transcripts (DETs) were obtained by pairwise comparisons of the samples (Fig. 1d, Extended Data Fig. 2b and Supplementary Table 1). We performed a K-means clustering analysis of DETs with $k=10$ (Fig. 1e,f and Extended Data Fig. 2c). Ten main clusters of expression patterns were discerned. We focused on cluster 8 and 9, which represented transcripts that are gradually downregulated during aging and included genes that are important in chromatin remodeling, such as $K d m 3 b, K d m 5 b, K m t 2 e$, and Park7 (Fig. 1e, Extended Data Fig. $2 \mathrm{~d}$ and Supplementary Table 2). Interestingly, histone H3K9me1/2 demethylase $K d m 3 b$ is correlated with the abnormalities of human eye, including nystagmus, low vision, and learning of cerebellum-dependent optokinetic response $(\mathrm{OKR})^{17}$. We confirmed that KDM3B mRNA and protein levels were approximately $25 \%$ decreased in 18 -month-old mice compared to that in 2-month-old mice (Fig. $1 \mathrm{~g}$, h). Furthermore, KDM3B was highly expressed in heterochromatin and chromocenters in 2-month-old mice, whereas KDM3B level decreased in heterochromatin and chromocenters in 18-month-old mice (Extended Data Fig. 3a). Collectively, these results suggest that KDM3B may be crucial for the regulation of heterochromatin feature in mice retina during aging.

Abnormal eye phenotypes in $\boldsymbol{K} \boldsymbol{d m} \mathbf{3} \boldsymbol{b}^{+-}$mice. To determine the cellular and molecular functions of KDM3B in the retina, we generated $K d m 3 b$ functional knock-out mice via the gene trap technique (Extended Data Fig. 3b, c, and Supplementary note). We confirmed that the KDM3B mRNA and protein levels were reduced by approximately $50 \%$ to $80 \%$ in $K d m 3 b^{+/-}$mouse retinas (Extended Data Fig. 3d, e, respectively). Additionally, publicly available mRNA-sequencing data (GSE 74660) of mouse retinal tissue showed that $K d m 3 b$ expression continued to increase up to stage P28 in whole retina and cones (Extended Data Fig. 3f). We found that KDM3B is highly enriched in the nucleus of cones, using immunohistochemistry (IHC), suggesting that it plays a role in the development or maintenance of cellular 
functions in cone photoreceptor cells (Extended Data Fig. 3g).

To demonstrate the functional role of $K d m 3 b$ in the eye, we observed the morphology and the structure of the enucleated eyeball and retinal tissue. We clearly detected smaller eyes and abnormal corneal phenotypes in 12-month-old $K d m 3 b^{+-}$mice compared to those in 1-month-old mice (Extended Data Fig. 3h). To investigate the structural and morphological differences in the retina, we measured the thickness of the entire retinas of 1-, 6-, and 12-month-old $K d m 3 b^{+/+}$and $K d m 3 b^{+/-}$mice. The retinal outer nuclear layer (ONL) of the $K d m 3 b^{+/}$mice was thinner than that of the $K d m 3 b^{+/+}$mice (Fig. 2a, and Supplementary Fig. 1a,b). The mean ONL thickness of $K d m 3 b^{+/}$mice was significantly reduced in 12 -month-old mice $(1,600$ $\mu \mathrm{m}$ inferior: $97 \pm 10 \mu \mathrm{m}$ versus $57 \pm 2.95 \mu \mathrm{m}, 1,600 \mu \mathrm{m}$ superior: $84.6 \pm 9.35 \mu \mathrm{m}$ versus $54.7 \pm 2 \mu \mathrm{m}$ ) (Fig. 2b). However, there was no difference between the mean ONL thickness of 1-month-old $K d m 3 b^{+/+}$and $K d m 3 b^{+/}$mice (Fig. 2a, b). Consistent with these results, we observed that the number of nuclei in the ONL was markedly reduced in $K d m 3 b^{+/}$mouse retinas among both 6-month-old (266 \pm 3.06 versus $239 \pm 2.34$ nuclei/100 $\left.\mu \mathrm{m}^{2}, P=1.93 \mathrm{E}-5\right)$ and 12-month-old mice $\left(239 \pm 3.38\right.$ versus $205.1 \pm 3.27$ nuclei $/ 100 \mu \mathrm{m}^{2}, P=$ 9.0E-6) (Fig. 2c). We also observed that the mean thickness of the inner nuclear layer (INL) and the number of nuclei in the INL and ganglion cell layer (GCL) were decreased in 1- and 6-month-old mice (Extended Data Fig. $4 \mathrm{a}, \mathrm{b})$. The decrease in ONL thickness in the $K d m 3 b^{+/}$retina could be because of reduced number of photoreceptor cell nuclei, including those of the rods and cones. To determine whether the rod and cone populations decreased in the ONL of the $K d m 3 b^{+/}$retina, we performed IHC with cone-cell specific (antiCAR; cone arrestin) and rod-cell specific (anti-RHO; rhodopsin) antibodies. We found that $K d m 3 b$ haploinsufficiency induced different morphologies and reduced the number of cones (14.6 \pm 0.41 versus $9.8 \pm 0.26$ cones $\left./ 100 \mu \mathrm{m}^{2} P=4.93 \mathrm{E}-34\right)$ in 12 -month-old mice, but it was not associated with differences in the rods (Fig. 2d, and Extended Data Fig. 4c).

Vertebrate rod and cone photoreceptors include outer segments (OS) and cell bodies that contribute to phototransduction, are required for cell viability, and a synaptic terminal able to signal to second-order neurons ${ }^{18}$. To determine the progression of cone loss in aged $K d m 3 b^{+/}$retinas, we examined the density of 
cones and the morphological changes of cone OS by immunostaining analysis of whole mouse retinas. Consistent with that of retina sections, the mean number of cones was significantly decreased in 12-monthold $K d m 3 b^{+/-}$mouse retinas, but the mean diameter of the cone-cell bodies was not significantly different between $K d m 3 b^{+/+}$and $K d m 3 b^{+/-}$mice (Fig. 2e, and Supplementary Fig. 1c,d). The mean length of the inner segment of cones was significantly decreased in 12-month-old $K d m 3 b^{+/}$mouse retinas (Fig. 2f). However, the mean circumference of the cone OS was similar between $K d m 3 b^{+/+}$and $K d m 3 b^{+/}$mouse retinas (Fig. 2f). Even though the overall mean cone lengths were similar, the mean lengths of the basal side and conecell body in the $K d m 3 b^{+/}$mouse retinas were longer than those of the $K d m 3 b^{+/+}$mouse retinas (Extended Data Fig. 4d). Interestingly, we observed that the mean presynaptic area of cones (pedicle) was smaller in the 12-month-old $K d m 3 b^{+/-}$mouse retinas (Fig. 2g, and Supplementary Fig. 1e). These results suggest that $K d m 3 b$ haploinsufficiency induced morphological changes and loss of cone photoreceptors in the aged mouse retina.

Transcriptomic profiling of $\mathbf{K} \boldsymbol{d m} 3 \boldsymbol{b}^{+/-}$mouse retinas. After determining the morphological changes in the $K d m 3 b^{+-}$mouse retinas, we analyzed differential transcript expression in $K d m 3 b^{+/-}$retina. We performed transcriptome profiling of 12 -month-old $K d m 3 b^{+/+}$and $K d m 3 b^{+/-}$mouse retinas by RNA-sequencing analysis. Principal component analysis (PCA) plot showed that $K d m 3 b^{+/-}$retinas accounted for the largest variance, and biological replicates showed great reproducibility (Fig. 3a). Volcano plots present the statistical significance of differential transcript expressions with respective fold-changes $(P<0.05$, absolute $\log _{2}$ fold-change $\left.\left(\log _{2} \mathrm{FC}\right)>0.5\right)$ compared to the expression observed in the control group (Fig. $3 \mathrm{~b}$ ). Application of DEseq with conservative access to the RNA-sequencing data gained from the $K d m 3 b^{+/-}$ retinal samples confirmed 3,978 differentially expressed transcripts (Fig. 3c and Supplementary Table 3). The significant upregulation or downregulation of approximately $20 \%$ genes was revealed in the $K d m 3 b^{+/-}$ retina (19,485 annotated transcripts, Extended Data Fig. 5a). Gene ontology (GO) analysis was performed to reveal the biological connection among the upregulated (1,814 genes) and downregulated (1,852 genes) 
DEGs in $K d m 3 b^{+/}$mouse retinas (Fig. $3 \mathrm{~d}$ and Supplementary Table 4). Clustering of the upregulated DEGs in $K d m 3 b^{+/-}$retinas enabled their classification into several categories that were associated with mRNA splicing and DNA damage stimuli, and downregulated DEGs were involved in synapse assembly and vesicle transport (Fig. 3d). Gene set enrichment analysis (GSEA), which shows the biological pathways and processes of the DEGs, also revealed that genes associated with cellular responses to DNA damage stimuli and apoptotic processes were upregulated, while the repressed genes in $K d m 3 b^{+/}$mouse retinas were involved in synapse assembly and vesicle-mediated transport (Fig. 3e). The selected genes in these groups were upregulated or downregulated in $K d m 3 b^{+/}$mouse retinas, indicating that KDM3B has a crucial role in cell death and synaptic transmission in the mouse retina (Fig. 3f, g). Since KDM3B haploinsufficiency induced the expression of gene sets for mRNA splicing, we also investigated alternative splicing events in RNA-sequencing data. The identified splice-site variants were categorized as 3,038 events for skipped exon (SE), 387 events for 5'-splice site (5'-SS), 642 events for 3'-splice site (3'-SS), 342 events for mutually exclusive exon (ME), and 906 events for retained intron (RI) (Extended Data Fig. 5b). These events showed the distribution of protein-coding genes (Extended Data Fig. 5c). RI is retained by the glycolytic enzyme, Aldolase A (Aldoa). The Aldoa transcript in $K d m 3 b^{+/+}$mice did not include any introns between exon 3 and exon 5; however, the introns were not spliced in the $K d m 3 b^{+/-}$mouse retinas, suggesting a novel effect of KDM3B in transcript maturation (Extended Data Fig. 5d). These results showed that KDM3B ablation selectively regulated the expression of gene sets involved in neuronal apoptosis and synaptic transmission.

Induction of apoptotic signals in aged $K d m 3 b^{+/-}$mouse retinas. Previous studies suggested that apoptosis occurs in pathologic photoreceptor cell death in several mouse models of retinal degeneration ${ }^{19}$. Since the number of cone photoreceptors was reduced in $K d m 3 b^{+/}$aged mice, we investigated whether retinal cells in the ONL were damaged due to the death of photoreceptors. We detected the presence of Müller glial cells, which are involved in the clearance of damaged cells and retinal regeneration, using anti-GFAP antibodies. The mean Müller cell counts were significantly increased in 6- and 12-month-old $K d m 3 b^{+/-}$retinas 
compared to that in age-matched $K d m 3 b^{+/+}$retinas (Fig. 4a, and Extended Data Fig. 6a). To further confirm whether retinal cell damage induces photoreceptor cell death through apoptosis, we stained 1- and 12month-old $K d m 3 b^{+/+}$and $K d m 3 b^{+/-}$retinas with the executive apoptotic marker, cytochrome c. The intensity of cytochrome c was significantly increased in 12 -month-old $K d m 3 b^{+/}$retinas compared to that in 1-monthold $K d m 3 b^{+/-}$retinas. However, this was not observed in 12- and 1-month-old $K d m 3 b^{+/+}$retinas (Fig. 4b). Interestingly, we observed an increased release of cytochrome $\mathrm{c}$ in the cones of 12-month-old $\mathrm{Kdm} 3 \mathrm{~b}^{+/-}$ retinas (Fig. 4c, and Supplementary Fig. 2a). Furthermore, we used TUNEL staining to determine whether degenerating cone photoreceptors underwent apoptosis through gDNA fragmentation. TUNEL-positive apoptotic cones also markedly increased in 12-month-old $K d m 3 b^{+/}$retinas (Fig. 4d, Extended Data Fig. 6b, and Supplementary Fig. 2b). Cytochrome c and TUNEL-positive signals were densely detected in the outermost ONL, where most of the cone photoreceptors are located in the retina (Fig. 4c, d). To explore the potential molecular mechanism that mediated KDM3B-induced apoptosis, we investigated the levels of apoptosis-associated proteins using a mouse apoptosis antibody array. Several apoptosis-associated proteins were upregulated or downregulated in 8-month-old $K d m 3 b^{+/}$retina (Fig. 4e, f, and Extended Data Fig. 6c, d). Compared to that in the $K d m 3 b^{+/+}$retina, the expression of pro-apoptotic molecules, such as Bad, increased by $20 \%$ in 8 -month-old $K d m 3 b^{+/}$retina (Fig. 4e). Therefore, $K d m 3 b$ haploinsufficiency upregulated apoptotic signals in cone photoreceptors.

Synaptic functional morphology of cone photoreceptors in $K d m 3 \mathbf{b}^{+-}$mouse retinas. Photoreceptor ribbon synapses are specialized synaptic structures in the outer plexiform layer, where visual signals are transmitted from photoreceptors to bipolar and horizontal cells ${ }^{20}$. Since functional KDM3B is required for the expression of synaptic and vesicle transport gene sets, we examined whether $K d m 3 b$ haploinsufficiency affected changes in the functional morphology of cone ribbon synapses. The mean area of cone synaptic terminals and the number of ribbon synapses was significantly reduced in 12 -month-old $K d m 3 b^{+/-}$retinas (Fig. 5a, Supplementary Fig. 3, and Supplementary video). Furthermore, we performed transmission 
electron microscopy (TEM) to elucidate the fine structure of photoreceptor synaptic regions. $K d m 3 b$ deficiency did not affect the length of ribbon synapses of both rods and cones (Extended Data Fig. 7a). The number and diameter of synaptic vesicles in the presynaptic regions of rod spherules and cone pedicles showed no differences between 12-month-old $K d m 3 b^{+/+}$and $K d m 3 b^{+-}$retinas (Fig. $5 \mathrm{~b}$ and Extended Data Fig. 7b). However, the mean number of docked vesicles of cone ribbon synapses decreased in 12-monthold $K d m 3 b^{+/}$retinas $(3.8 \pm 0.35$ versus $2.9 \pm 0.27, P=0.0285)$ (Fig. $\left.5 \mathrm{c}\right)$. These findings show that KDM3B is required for the synaptic components and the visual acuity associated with cone photoreceptors.

Electrophysiological synaptic impairment of cone photoreceptors in $K d m 3 b^{+/-}$mouse retinas. To investigate the physiological role of KDM3B in vivo, we compared electroretinograms (ERGs) of 3-, 6- and 12-month-old $K d m 3 b^{+/+}$and $K d m 3 b^{+/}$mice under dark-adapted (scotopic) and light-adapted (photopic) conditions. The amplitude of the scotopic ERG a-wave and b-wave reflects phototransduction in the OS of rods and rod bipolar cells and that of photopic ERGs, reflects both phototransduction in the OS of cones and cone bipolar cell activity ${ }^{21}$. Under scotopic conditions, the amplitude of a-waves and b-waves, as well as rod-mediated outer retinal activity were comparable between 3-, 6-, and 12-month-old $K d m 3 b^{+/+}$and $K d m 3 b^{+/}$mice, indicating that rod function was not significantly affected (Fig. 5d-f, and Extended Data Fig. 7c-e). Under photopic conditions, the a-wave amplitude was not significantly different at both low and high stimuli in 3-, 6-, and 12-month-old mouse retinas (Fig. 5g, h and Extended Data Fig. 7f-h). However, photopic b-waves were significantly reduced in 6-month-old $K d m 3 b^{+/-}$mice compared to those in $K d m 3 b^{+/+}$ mice at higher stimulus luminance $\left(194 \pm 43.8\right.$ versus $129.6 \pm 41.9 \mu \mathrm{V}$ at $\left.1.2 \log \cdot \mathrm{cd} \mathrm{sec} / \mathrm{m}^{2}, P=0.0079\right)$, which may be attributable to the impaired synaptic transmission between cones and bipolar cells (Fig. $5 \mathrm{i}$ and Extended Data Fig. 7i). These results suggest that synapse transmission from cone photoreceptors to cone bipolar cells was impaired in 6-month-old $K d m 3 b^{+/}$mice.

Site-specific regulation of $\mathrm{H3K9}$ methylation patterns by KDM3B in mouse retinas. Because KDM3B 
is required for the physiological and functional roles of cones, we performed ChIP-seq analysis to determine whether KDM3B was directly involved in gene expression via histone demethylase activity. Over 32 million reads were obtained for KDM3B ChIP-seq; these were aligned with the mouse reference genome (GRCm 38.99), and peak calling was performed (Supplementary Table 5). The majority of KDM3B peaks were globally found within $100 \mathrm{~Kb}$ from the transcription start site (TSS) (Fig. 6a). The distribution of KDM3B-binding peaks signified that the majority of KDM3B was localized in intergenic regions $(50 \%)$ and introns (42\%) (Fig. 6b). To further test the functional importance of KDM3B in gene expression regulation, we performed the GO analysis of the KDM3B-binding peaks. Interestingly, the KDM3B ChIPseq signals were highly enriched for genes involved in nervous system development, such as synapse assembly and organization, neuron generation, and differentiation (Fig. 6c and Supplementary Table 6).

Previous studies have determined that KDM3B has demethylase activity for $\mathrm{H} 3 \mathrm{~K} 9 \mathrm{mel}$ and H3K9me2 but not for H3K9me3 ${ }^{9,22}$. To determine whether KDM3B associated with H3K9me1, H3K9me2, majority of $\mathrm{H} 3 \mathrm{~K} 9 \mathrm{me} 1, \mathrm{H} 3 \mathrm{~K} 9 \mathrm{me} 2$, and $\mathrm{H} 3 \mathrm{~K} 9 \mathrm{me} 3$ peaks were globally found within $100 \mathrm{~Kb}$ from the TSS, and most $\mathrm{H} 3 \mathrm{~K} 9 \mathrm{me} 1, \mathrm{H} 3 \mathrm{~K} 9 \mathrm{me} 2$, and $\mathrm{H} 3 \mathrm{~K} 9 \mathrm{me} 3$ peaks were localized in intergenic regions and introns

and $\mathrm{H} 3 \mathrm{~K} 9 \mathrm{me} 3$ marks the same target genes, we performed $\mathrm{H} 3 \mathrm{~K} 9 \mathrm{me} 1, \mathrm{H} 3 \mathrm{~K} 9 \mathrm{me} 2$, and $\mathrm{H} 3 \mathrm{~K} 9 \mathrm{me} 3 \mathrm{ChIP}-\mathrm{seq}$ using 2-month-old $K d m 3 b^{+/+}$and $K d m 3 b^{+/}$mouse retinas. Similar to the results of KDM3B enrichment, the (Extended Data Fig. 8a, b). Consistent with the results of previous reports ${ }^{9,22}$, KDM3B haploinsufficiency resulted in a dramatic increase in $\mathrm{H} 3 \mathrm{~K} 9 \mathrm{me} 1$ and $\mathrm{H} 3 \mathrm{~K} 9 \mathrm{me} 2$ levels, while the global patterns of $\mathrm{H} 3 \mathrm{~K} 9 \mathrm{me} 3$ levels were not significantly different between $K d m 3 b^{+++}$and $K d m 3 b^{+-}$mouse retinas (Fig. 6d). Next, all H3K9me1, H3K9me2 and H3K9me3 peaks were categorized into three clusters: present in both $\mathrm{Kdm} 3 \mathrm{~b}^{+/-}$ and $K d m 3 b^{+/+}$mice (cluster 1), present only in $K d m 3 b^{+/}$mice (cluster 2), and present only in $K d m 3 b^{+/+}$ mice (cluster 3). Centralization of ChIP-seq peaks indicated that H3K9me2 levels in cluster 2 increased only in $\mathrm{Kdm}_{3} \mathrm{~b}^{+/-}$retinas, indicating that $\mathrm{H} 3 \mathrm{~K} 9 \mathrm{me} 2$ levels are regulated in a KDM3B activity-dependent manner. Also, the enrichment of $\mathrm{H} 3 \mathrm{~K} 9 \mathrm{me} 2$ in cluster 3 proceeded as a demethylase-independent function of KDM3B (Fig 6e). The scatter-density plot of the H3K9me2 level at the TSS between $K d m 3 b^{+/+}$and 
$K d m 3 b^{+/}$mouse retinas revealed a markedly negative correlation (Fig. 6f). Furthermore, using GO analysis, we found that the $\mathrm{H} 3 \mathrm{~K} 9 \mathrm{me} 2$ enriched target genes in $K d m 3 b^{+/}$retinas (cluster 2), indicating that repressed genes are involved in synaptic signaling, synapse organization, and chemical synaptic transmission (Fig. $6 \mathrm{~g}$ ). On the other hand, the $\mathrm{H} 3 \mathrm{~K} 9 \mathrm{me} 2$ peaks in $K d m 3 b^{+/+}$(cluster 3 ) were enriched for negative regulation of apoptosis and signal transduction (Fig. 6g). We obtained similar results for the global pattern of H3K9me1 peaks and biological processes by GO analysis (Fig. 6h-j). Together, these ChIP-seq analyses demonstrated that $\mathrm{KDM} 3 \mathrm{~B}$ is associated with $\mathrm{H} 3 \mathrm{~K} 9 \mathrm{me} 1$ and $\mathrm{H} 3 \mathrm{~K} 9 \mathrm{me} 2$, suggesting that $\mathrm{KDM} 3 \mathrm{~B}$ exclusively regulates gene sets between neuronal homeostatic genes (synaptic transmission, vesicle transport) and cell-death related genes.

\section{KDM3B upregulates the expression of synaptic genes through the erasure of H3K9me1 and}

H3K9me2 signatures. The balance between histone methylation and demethylation is highly correlated with transcriptional regulation. We determined the molecular mechanism by which KDM3B reduced the stimulation of synapse assembly genes in $K d m 3 b^{+/}$mice by integrating ChIP-seq and RNA-sequencing analysis. Approximately 31\% (569 genes) and 43\% (803 genes) of upregulated or downregulated DEGs, respectively, overlapped with all KDM3B ChIP-seq peaks (Fig. 7a, b). Consistent with RNA-sequencing analysis, GO analysis showed that genes bound by KDM3B and upregulated genes were involved in DNA damage stimuli and transcription regulation, while downregulated genes in $K d m 3 b^{+/}$mice were exclusively enriched for neuronal development, synapse assembly, and vesicle-mediated transport (Fig. 7c-f and Supplementary Table 7). We next focused on the 803 downregulated genes in $K d m 3 b^{+/}$mice to determine whether gene expression was modulated by the histone demethylation activity of KDM3B. H3K9me1 and H3K9me2 levels were significantly increased in association with downregulated genes in $\mathrm{Kdm} 3 \mathrm{~b}^{+/-}$mice, revealing the deposition of histone methylation due to the ablation of KDM3B enzymatic functions (Fig. 7g). Additionally, H3K9me3 level was slightly increased in some sets of downregulated genes (Fig. 7g). KDM3B was enriched in the synapse assembly genes, including Nrxn2 and Rims2, which were 
downregulated in $K d m 3 b^{+/}$mice (Fig. 7h). Based on ChIP-seq data, we selected ten target genes, such as synapse assembly genes and apoptosis-induced genes for validation by ChIP-qPCR. KDM3B was enriched in the target gene promoters (within $\sim 2 \mathrm{~Kb}$ from TSS) representing the normal range of ChIP-seq peaks for $\mathrm{H} 3 \mathrm{~K} 9 \mathrm{me} 1$ and $\mathrm{H} 3 \mathrm{~K} 9 \mathrm{me} 2$ in $K d m 3 b^{+/+}$, and the signal was significantly elevated in $K d m 3 b^{+/}$mouse retinas (Fig. 7i, and Extended Data Fig. 9, 10). These results suggest that KDM3B modulated the transcriptional

KDM3B associates with AP-1 transcription factors. Since KDM3B does not directly bind to DNA, KDM3B might be associated with DNA through binding to specific transcription factors. In leukemogenesis, KDM3B interacted with CBP, forming an activator complex during 1 mo2 transcription activation'. To dynamics of synaptic and apoptotic genes by balancing the H3K9 methylation status.

identify potential new partners of KDM3B, we analyzed de novo motif discovery using the MEME-ChIP algorithm. We identified a list of 10 enriched motifs (Supplementary Table 8). Two highly enriched de novo motifs showed significant similarity to a known ZNF384 (motif enrichment E-value $=3.45 \mathrm{e}-05$ ) and transcription factor of AP-1 family (motif enrichment E-value = 1.16E-04) (JASPAR database) (Extended Data Fig. 11a). Furthermore, we analyzed the positional distribution of ZNF384 and c-Jun enriched motifs using the Centrimo algorithm. Both the ZNF384 and c-Jun motifs were centrally enriched with respect to the KDM3B-ChIP peaks (Fig. 8a). De novo motif analysis of the KDM3B ChIP-seq regions yielded potential binding regions for transcription factors that might co-occupy KDM3B-regulated genomic sites and potentially modulate its target genes.

Since the AP-1 family regulates the various cellular processes, including cell proliferation, differentiation, and apoptosis ${ }^{23}$, it is reasonable to speculate that KDM3B modulates neuronal homeostatic genes and cell death-related genes by interacting with AP-1 transcription factors. To test this hypothesis, we first confirmed that AP-1 family (c-Fos, c-JUN, and JUNB) mRNA and protein levels were stably expressed up to 12 months in mouse retina (Extended Data Fig. 11b, c). Transcriptome profiling showed that $c$-Fos and $J U N B$ mRNA levels increased in $K d m 3 b^{+/}$mice retina (Extended Data Fig. 11d). In addition, 
public mRNA-sequencing data (GSE 74660) from mice retina tissue showed that the expression of $c$-Fos, $c-J U N$ and $J U N B$ genes was higher in cone than in rod photoreceptors (Extended Data Fig. 11e). Next, we analyzed whether AP-1 transcription factors were involved in KDM3B recruitment in vivo. Finally, coimmunoprecipitation experiments showed that KDM3B was associated with c-Fos and JUNB in mice retina and human lung cancer cells (Fig. 8b and Extended Data Fig. 11f). Additionally, ChIP-qPCR with c-Fos and JUNB antibodies confirmed that both transcription factors were associated with KDM3B target genes (Fig. 8c, and Extended Data Fig. 11g). These results suggest that KDM3B, c-Fos, and JUNB can physically interact with and could regulate a set of KDM3B target genes.

$K d m 3 b$ haploinsufficiency accelerated the destruction of epigenomic network robustness. To determine the difference between $K d m 3 b^{+/+}$and $K d m 3 b^{+/}$networks, we constructed KDM3B-centered epigenomic networks using integrated RNA-sequencing and ChIP-Seq analysis. The $K d m 3 b^{+/+}$network consisted of 1,452 genes with 2,906 links, while the $K d m 3 b^{+/}$network consisted of 1,376 genes with 2,384 links (Fig. 8d). The clustering coefficient $\left(K d m 3 b^{+/+}=0.18\right.$ versus $\left.K d m 3 b^{+/}=0.16\right)$ and the diameter of the nodes were similar between the $K d m 3 b^{+/+}$and $K d m 3 b^{+/}$networks, indicating a lack of a significant difference between the global properties of the networks. To strictly measure the structural differences, the network dissimilarity concept $\left(D\left(G, G^{\prime}\right)\right)^{24,25}$ was introduced to the expanded network for four different ages of $K d m 3 b^{+/+}$mouse retinas $\left(1.5,3,6\right.$, and 12 months) compared to those of $K d m 3 b^{+/}$mouse retinas. The $D$-value among the four different ages of $K d m 3 b^{+/+}$mice showed relatively small dissimilarities, from 0.001 to 0.012 (Fig. 8e). In contrast, there was a significant difference in the mean $D$-value between $K d m 3 b^{+/+}$and $K d m 3 b^{+/}$networks $(0.032 \pm 0.001$ at 12 months) (Fig. 8e). This indicated that the network dissimilarity was clear between the 12-month-old $K d m 3 b^{+/+}$and $K d m 3 b^{+/}$mouse retinas.

Network robustness is a central question in systems biology and medicine, helping us understand how subtle changes or failures lead to the development of disease ${ }^{26}$. Therefore, we measured the network 
robustness between the $K d m 3 b^{+/+}$and $K d m 3 b^{+/}$genotypes by random link removal. We simulated random

361 link removal with a fraction $f$ and measured the fraction of the largest cluster size $\left(P_{\infty}(f) / P_{\infty}(0)\right)$ as an 362 order parameter (Fig. 8f). Since all epigenomic networks of KDM3B are based on centralized KDM3B 363 networks and finite-sized networks, we can predict that the critical threshold $f_{c}$ goes to $1^{27}$. Therefore, we 364 focused on the difference of $P_{\infty}(f) / P_{\infty}(0)$ for various values of $f$. The order parameters of all four $365 K d m 3 b^{+/+}$network stages showed more robustness for link failure than those of $K d m 3 b^{+/}$(Fig. 8f). These 366 results imply that the $K d m 3 b$ haploinsufficiency causes epigenomic network dissimilarity, resulting in the acceleration of the destruction of network robustness. 
Aging is a complex multifactorial biological process shared by all Metazoa. Organismal aging holds significance for human health because it increases susceptibility to many diseases, including cancer, diabetes, cardiovascular disorders, and neurodegenerative diseases. Environmental factors are the major effectors that increase or decrease life expectancy. Environmental conditions may affect the cellular and molecular epigenomic modifications including "loss of heterochromatin" to regulate gene expression and cell fate, which result in the aggravation or alleviation of the aging process.

Retinal aging is often associated with a decrease in visual acuity, ocular accommodation, and dark adaptation. Additionally, this aging phenotype is aggravated by several diseases, such as AMD. Recently, many researchers have investigated epigenetic changes, including DNA methylation and histone modification in retinal homeostasis. During the development of the vertebrate retina, multipotent progenitors differentiate into various neuronal subtypes as a result of gene expression regulation ${ }^{28,29}$. For retinal development, the appropriate regulation of histone methylation is important for cell proliferation and differentiation? ${ }^{7}$. Histone H3K9 methyl transferase G9a (Ehmt2), mainly present in retinal progenitor cells, plays an essential role in proper retinal development by repressing the expression of progenitor genes in terminal differentiation ${ }^{30}$. Kdm6b, which encodes H3K27 demethylase (JMJD3), is required for the survival of rod bipolar cells with the regulation of Bhlhb4 expression ${ }^{31}$. Histone demethylase Kdm5b, known as histone $\mathrm{H} 3 \mathrm{~K} 4$ demethylase, functions as a regulatory factor for rod-specific genes in the retina ${ }^{32}$. Our study demonstrated that KDM3B is a putative regulator of apoptotic signaling and synaptic transmission, playing a balancing role in the retinal aging process. Moreover, instead of global heterochromatin loss, we found that KDM3B selectively modulates the heterochromatin features in a genefunction dependent manner, i.e., differently for apoptotic or neuro-functional genes. The synapses were also altered in cellular morphology and structure by downregulation of $K d m 3 b$ expression, indicating that $K d m 3 b$ haploinsufficiency disturbs retinal homeostasis. Screening the expression of apoptosis-related proteins in $K d m 3 b^{+/}$mice retinas revealed the upregulation of pro-apoptotic protein Bad and the 
downregulation of anti-apoptotic protein Catalase (Fig.4e). Bad is a member of the Bcl-2 family, which was identified as an intersection point between promotion and inhibition of apoptosis ${ }^{33}$. Catalase protects against oxidative stress-induced toxicity ${ }^{34}$. Finally, dysregulated retinal homeostasis induces damage to cone photoreceptors and initiates cell death in $K d m 3 b^{+/}$mouse retinas.

AMD is characterized by reduced retinal pigment epithelium (RPE) function and photoreceptor loss in the macular area. Genetic variant studies of AMD can help uncover disease mechanisms and provide entry points into its therapy. Despite genetic studies investigating AMD, there is a limited understanding of the underlying physiological and molecular pathogenetic mechanisms and therapies of this complex disease. AMD pathogenesis is triggered by genetic and environmental factors ${ }^{35}$. In particular, epigenetic alteration is an important factor for gene regulation without genetic variation. Of the environmental risk factors that have been investigated, smoking may be associated with a two-fold increased risk of developing $\mathrm{AMD}^{36}$. Therefore, the integration between genetic and epigenomic studies may contribute to more effective functional analyses of the mechanisms underlying AMD pathogenesis. Previous evidence supports the role of aberrant epigenetic modifications with significant increase in the mRNA expression of HDAC1, HDAC3, HDAC6, DNMT1, and DNMT3a in the RPE cells of mice with excessive iron levels, which are, thus, at a higher risk of developing $\mathrm{AMD}^{37}$. A recent study showed KDM3B SNPs caused eye abnormalities (31\%, $5 / 16$ cases), such as nystagmus and strabismus in four individuals, as well as refraction anomalies and low vision in three individuals ${ }^{38}$. We generated heterozygous $K d m 3 b$ mice and focused on the function of KDM3B in the mouse retina via physiological, genetic, and multi-dimensional epigenomic analysis. We found that $K d m 3 b$ mRNA expression is similar between rods and cones during development, but the protein level is highly enriched only in cones (Extended Data Fig. 3). Thus, KDM3B has cell type specificity and different functional roles at the protein level. ONL thickness and cone photoreceptor populations can be reduced in $K d m 3 b^{+/}$mouse retina by regulating apoptotic genes (Fig. 2 and 4). Furthermore, KDM3B plays an important role in synapse assembly and vesicle transport in cone ribbon synapses through multidimensional epigenomic network analysis (Fig. 8d-f). We suggest that the cone-cell type specificity and 
KDM3B functional network can cause age-related diseases, such as AMD. Future studies will demonstrate whether KDM3B is associated with cone-cell type specificity in the human retina.

The duplex retina in vertebrates constitutes specialized light-sensitive rod and cone photoreceptors $^{39}$. Rods permit energy conservation and maximum sensitivity at the expense of spatial and temporal resolution, whereas cones enable non-quenching, rapid responses to photons with high acuity in daylight ${ }^{40}$. Rods and cones coordinate synaptic connections with bipolar and horizontal cells for visual transmission. Previous studies have shown the important roles of epigenetic regulation in retinal development and homeostatic maintenance. For instance, the histone H3K4 methyl transferase MLL1, is essential for retinal structure, functional synapse formation, horizontal cell differentiation, and maintenance ${ }^{41}$. Ablation BMI1, a component of the PRC1 histone H3K27 methyl transferase complex, leads to increased retinal cell death in bipolar and cone cells ${ }^{42}$. Despite these observations, the exact role of epigenetic regulation in photoreceptor development and degeneration is largely unknown. We demonstrated the functional morphology and physiology of cone synapses in the $K d m 3 b^{+/-}$retina. Reduced ERG waves in $K d m 3 b^{+/}$mice imply defective phototransduction in cones. A higher decrease in B-waves than in Awaves in photopic ERGs suggests abnormalities in visual transmission in cones. The number of cone ribbon synapses and docked vesicles in cone pedicles were reduced in $K d m 3 b^{+-}$mice (Fig. 5). Additionally, $K d m 3 b^{+/}$mouse retinas showed that the synapse assembly genes including Rims2, Nrxn2, CtBP2, Nedd4, and vesicle-transport genes, including Tmed10, Vamp4 were downregulated by increasing H3K9 methylation (Fig. 7). This suggests that the presynaptic terminal abnormalities in cones proceed from epigenetic modification complications followed by incorrect assembly of ribbon synapses.

Rising evidence suggested that epigenetic modulators often exhibit dual function, both activator and repressor, in gene transcriptional regulation, development, and cancer progression. However, how these dual functions are coordinated in specific cellular contexts remains poorly understood. Therefore, it is interesting that KDM3B, an active histone demethylase, unexpectedly activates apoptotic genes in aged $K d m 3 b^{+/}$mice retinas. Since KDM3B lacks DNA binding ability, it may require a transcription factor for 
gene-set specific regulation. Consistent with this hypothesis, our RNA-sequencing analysis supported an increasing expression pattern of AP-1 family transcription factors during retinal aging. In addition, motif analysis of KDM3B ChIP-seq data uncovered a list of transcription factors that were associated with specific target genes. In particular, KDM3B peaks carried a motif predicted by AP-1 family transcription factors such as c-Fos and JUNB. We demonstrated that KDM3B co-occupies a subset of KDM3B target genes via physical interaction; however, it remains to be determined if this interaction is direct or indirect

(Fig. 8). The immediate early gene $c$-Fos is expressed in various cell types by numerous stimuli and conditions. C-Fos is well known to dimerize with c-JUN family proteins, which are a major component of the AP-1 transcriptional complex. Previous reports indicate that c-Fos is implicated in the induction of cell death in several types of neuronal cells. c-Fos is required for both regeneration of retina ganglion cells and apoptotic cell death ${ }^{43,44}$. Consistent with the results from previous studies, we observed that c-Fos and JUNB are associated with KDM3B at the regulatory regions of apoptosis-induced genes and synapse assembly are yet to be elucidated, our data shed some light on novel aging regulatory mechanisms.

GRNs affect all biological phenomena associated with maturation (development), rapid genes in mice retina. This finding further supports the assertion that c-Fos and JUNB might fine-tune the transcription of KDM3B target genes acting as on-off switches during aging. Although detailed mechanisms destruction (disease), and slow destruction (aging) (Fig. 8g). In terms of network topology, conventional GRNs cannot differentiate between subtle changes, such as environmental adverse effects and aging. We suggest that the integration of multi-dimensional epigenomic networks and GRNs will help improve the understanding of biological phenomena by allowing for the measurement of network strength during aging. We focused on an epigenetic regulator (KDM3B)-centered network during retinal aging. Even though many studies have investigated epigenetic regulation in cancers or stem cells, the precise mechanism of epigenomic modification in completely differentiated and aging cells is largely unknown. KDM3B is an H3K9me1 and H3K9me2 demethylase that belongs to the KDM3 family and acts as a transcriptional activator $^{9,45}$. KDM3B accelerates leukemogenesis by regulating $1 \mathrm{mo} 2$ expression via the reduction of 
H3K9me2 methylation ${ }^{22}$. Additionally, the demethylation activity of KDM3A/B activates Wnt target genes, which increase the survival of human colorectal cancer stem cells ${ }^{22}$. Previous reports implicate H3K9 methylation remodeling in aging. Histone methyl transferase SUV39H1 decreases in line with age, resulting in a reduction of global H3K9me level and disturbed heterochromatin function ${ }^{46}$. Another report showed that the disruption of Kdm4A causes shortening of the lifespan of male Drosophila, indicating that KDM4A is involved in longevity ${ }^{47}$. We established $K d m 3 b^{+/}$mice and demonstrated the functional roles of KDM3B via morphological and physiological changes, as well as genome-wide transcriptional and epigenomic analysis during retinal aging. Importantly, our data suggest that site-specific changes of H3K9 methylation in $K d m 3 b^{+/}$mice accelerate the destruction of GRN leading to "loss of heterochromatin features" affecting cellular lifespan depending on organism. Furthermore, we constructed the KDM3B-centered GRN using integrated RNA-Sequencing and ChIP-Seq analysis, which showed that the strength of the network between a transcriptional regulator and its target genes weakened in $K d m 3 b^{+/}$mice. Although the network structural properties are not significantly different between $K d m 3 b^{+/+}$and $K d m 3 b^{+/-}$mice (Fig. 8d), we found interior topological differences by measuring network dissimilarity and robustness (Fig. 8f). Eventually, $K d m 3 b$ haploinsufficiency diminished the structural gene network via heterochromatin misregulation and induced cascading destruction of network integrity; these delicate alterations emerged as post-transcriptional and translational regulations. For example, disrupted alternative splicing of the $C P S F 1$ and $C N O T 3$ genes leads to the attenuation of biological processes in retinitis pigmentosa $(\mathrm{RP})^{48}$. We identified the retained intron events on the Aldoa gene in $K d m 3 b^{+/-}$mouse retinas (Extended Data Fig. 5d). ALDOA is a glycolytic enzyme that catalyzes the reversible conversion of fructose-1, 6-bisphosphate to glyceraldehyde 3phosphate and dihydroxyacetone phosphate ${ }^{49}$. This result suggests that $K d m 3 b$ haploinsufficiency changes the normal transcriptome profile, which is a potential cause of retinal disease via energy metabolism regulation during aging.

In summary, our findings provide new insight into how site-specific epigenetic regulation contributes to the maintenance of retinal homeostasis during aging. We demonstrated the presence of an 
493 epigenomic network in the retina, which might illuminate the selective roles of KDM3B in synaptic 494 transmission and apoptotic signaling pathways during retinal aging. We integrated epigenomic network 495 analysis with physiological and morphological analyses of the $K d m 3 b^{+-}$mouse retina. Ablation of $K d m 3 b$ 496 results in presynaptic cone abnormalities by inducing apoptotic signals. Kdm3b haploinsufficiency 497 downregulates a subset of synapse assembly genes, with H3K9 methylation-associated silencing. Overall, 498 these findings suggest that integrating genomic and epigenomic data in network studies can help to predict 499 whether subtle changes in network strength increase susceptibility to age-related neuronal disease. 
Animals. $K d m 3 b^{+/}$mice were developed by a gene trapping system using pGT0lxf plasmid inserted between exons 12 and 13 of the $K d m 3 b$ gene. $K d m 3 b^{+/-}$genotype was confirmed by PCR using genotyping primers: common forward, 5'-GGC ACC AGA CCC TGG GAG CTA G-3'; WT reverse, 5'-CAC CCA CGA CCT GGC TTA CAC C-3'; and KO reverse 5'-CAC CCA CGA CCT GGC TTA CAC C-3'. Mice were maintained in a 12-h light-dark cycle (lights were turned off at 20:00) with free access to water and mouse feed. The mice were weaned at the age of 3 weeks and housed 1-6 per cage. Mice were approved by the Institutional Review Board of Chung-Ang University (updated IRB number: 2019-00059 and 2020$00061)$.

Immunohistochemistry (IHC). $K d m 3 b^{+/}$mice retinas were excised quickly by removing the lenses on a cold plate submerged in PBS. After fixation of retinal tissues with 4\% paraformaldehyde (\#15710; Electron Microscopy Sciences, Hatfield, PA, USA), they were incubated successively in 10\%, 20\%, 30\% sucrosePBS for 1, 3, and $12 \mathrm{~h}$, each. The fixed retina was embedded with 7\% agarose gel or OCT compound (4583, SAKURA, CA, USA), and the retinas were sectioned with vibratome (7000SMZ, Campden Instruments, England) and cryotome (CM1850, Leica, Germany). After permeabilization in PBS with $0.1 \%$ Triton X100 for $10 \mathrm{~min}$, the sections were incubated for $1 \mathrm{~h}$ in blocking solution, which included 5\% normal goat serum in PBS with $0.1 \%$ Triton X-100. Primary antibodies were incubated overnight at $4^{\circ} \mathrm{C}$. Secondary antibodies were incubated for $1 \mathrm{~h}$ at $23-25^{\circ} \mathrm{C}$ in the dark after being washed with PBS. The list of antibodies is presented in Supplementary Table 9. The nucleus was counterstained with $5 \mu \mathrm{g} / \mathrm{ml} 40$, 60-diamidino-2phenylindole (DAPI) for $3 \mathrm{~min}$ in the dark. Fluorescence images were generated with Zeiss Axio Observer Z1 LSM 700 confocal microscope and ZEN program (ZEN lite 2011) (Carl Zeiss, Oberkochen, Germany). 
and $1 \mathrm{mM}$ PMSF. The cell suspensions of retina tissue were homogenized on ice and centrifuged at 15,000 $g$ at $4^{\circ} \mathrm{C}$ for $10 \mathrm{~min}$. For immunoprecipitation assays, the supernatants were precleaned with $20 \mu 1$ of protein $\mathrm{A} / \mathrm{G}$ magnetic agarose beads (50\% slurry) and then incubated at $4^{\circ} \mathrm{C}$ overnight with $40 \mu \mathrm{l}$ of protein $\mathrm{A} / \mathrm{G}$ magnetic agarose beads in the presence of appropriate antibodies. The beads were washed 3 times in PBS, resuspended in SDS sample buffer, and boiled for $10 \mathrm{~min}$. The protein samples from the retina were electrophoresed on a 10\% SDS-PAGE and transferred to a nitrocellulose membrane (Protran ${ }^{\mathrm{TM}}$; Whatman, Maidstone, UK). The membrane was blocked with 5\% skim milk in TBS-T buffer (137 mM NaCl, $20 \mathrm{mM}$ Tris- $\mathrm{HCl}$, pH 7.6 and $0.1 \%$ Tween-20) and incubated with suitable diluted primary antibody overnight at $4^{\circ} \mathrm{C}$. The list of antibodies is presented in Supplementary Table 9. The membranes were washed thrice for 10 min each with TBST and incubated with a 1:5,000 dilution of horseradish peroxidase-conjugated antimouse or anti-rabbit antibodies for $1 \mathrm{hr}$. Blots were washed with TBST three times and developed with the Western blotting luminol reagent (sc-2048, Santa Cruz), according to the manufacturer's protocols.

Total RNA isolation and RT-qPCR. Total RNA was extracted using the TRIzol solution (15596018, Invitrogen, CA, USA) according to the manufacturer's specifications. Contaminated genomic DNA was removed from $10 \mu \mathrm{g}$ of total RNA by incubation with 20 units of Rnase-free Dnase I (New England Biolabs) and 4 units of RNase inhibitor (New England Biolabs) in DEPC-treated water. The reaction mix was incubated for $1 \mathrm{hr}$ at $37^{\circ} \mathrm{C}$ and then for $10 \mathrm{~min}$ at $50^{\circ} \mathrm{C}$. RNA samples were quantified spectrophotometrically at $260 \mathrm{~nm}$ and all RNA extracts had an $\mathrm{OD}_{260}: \mathrm{OD}_{280}$ between 1.8 and 2.0, demonstrating that RNA was extracted clearly. Oligo-dT (6110A, Takara) was applied as the primer in the first step of cDNA synthesis. Total RNA $(1 \mu \mathrm{g})$ was combined with $1 \mu \mathrm{l}$ of oligo dT and $\mathrm{H}_{2} \mathrm{O}$ and then preheated at $70^{\circ} \mathrm{C}$ for $10 \mathrm{~min}$ to denature the secondary structures of RNA. The mix was then quickly cooled to $4^{\circ} \mathrm{C}$, then $10 \mathrm{mM}$ DTT, $2 \mu \mathrm{l}$ of $10 \mathrm{X}$ reverse-transcriptase buffer, and 200 units of reverse transcriptase (18064022, Invitrogen, CA, USA) were added to make a total volume of $20 \mu$. The reverse-transcriptase mixture was incubated at $40^{\circ} \mathrm{C}$ for $60 \mathrm{~min}$, subsequently the reaction was stopped by heating at $94^{\circ} \mathrm{C}$ for 
$20 \mathrm{~s}$. The cDNA stock was stored at $-20^{\circ} \mathrm{C}$.

The specificity of each of the amplified products was confirmed by melting curve analysis. For real-time quantitative PCR, the iQ SYBR Green PCR Supermix (\#1708880, Bio-Rad) and the CFX96 Real-time PCR detection system (Bio-Rad) were used to detect amplified cDNA samples according to the manufacturer's instructions. The $\beta$-actin gene was used for normalization. The relative mRNA expression was calculated by the $2^{-(\Delta \Delta C t)}$ method.

ERG analysis. Mice were given full field flash ERG to assess retinal function under scotopic and photopic conditions ${ }^{50}$. Mice were adapted to dark overnight for scotopic measurements and anaesthetized with $2 \mathrm{X}$ avertin solution (200 $\mu$ l per 20 g mouse) with tribromoethanol (T48402, SIGMA) and tert-amyl alcohol (240486, SIGMA). Their pupils were dilated with isopto atropine (Alcon, Republic of Korea), and a small drop of $1 \%$ hypromellose (Samil, Republic of Korea) was added to each eye before measurements. Ground and reference subdermal electrodes were placed subcutaneously near the hindquarter and between the eyes, respectively and the mice were placed on heated pads $\left(37^{\circ} \mathrm{C}\right)$. Recording electrodes were placed on the cornea, and ERG was carried out under scotopic conditions for both eyes simultaneously, with increasing green light stimulus strengths. After the mice adapted to $1.6 \log \mathrm{cd} \cdot \mathrm{sec} / \mathrm{m}^{2}$ for $5 \mathrm{~min}$, the photopic responses were recorded.

Mouse apoptosis antibody array. The expression of apoptosis-related proteins in mice retinas was analyzed suing a Mouse Apoptosis Array Kit (R\&D Systems, Minneapolis, MN, USA). All procedures were performed according to manufacturer's instructions. Nitrocellulose-membrane sheets containing 21 apoptosis-related proteins were incubated with blocking buffer at room temperature for $1 \mathrm{~h}$, then the mouse retina lysates were incubated overnight with the membranes at $4^{\circ} \mathrm{C}$. After rinsing, the array was incubated with a cocktail of biotinylated detection antibodies at room temperature for $1 \mathrm{hr}$ and probed with HRPconjugated streptavidin for $30 \mathrm{~min}$. A signal produced at each capture spot corresponding to the amount of 
protein bound was detected. Relative protein levels were estimated by comparing the pixel densities of protein spots and were quantified using ImageJ.

Transmission electron microscopic (TEM) analyses. Mice retinas were enucleated and fixed in $4 \%$ paraformaldehyde overnight at $4^{\circ} \mathrm{C}$. After three washes with $0.1 \mathrm{M}$ sodium cacodylate buffer, $\mathrm{pH} 7.2$, they were fixed in $1 \%$ osmium tetroxide/ $0.1 \mathrm{M}$ cacodylate buffer. The retinas were dehydrated using an ethanol gradient up to $100 \%$, and embedded in epoxy resin. Ultrathin sections were prepared using an ultracut diamond knife and stained with $2 \%$ uranyl acetate and $4 \%$ lead citrate. Specimens were visualized with a TEM (Tecnai F20 G2).

RNA sequencing and bioinformatic analysis. RNA-sequencing library was generated by TruSeq mRNA Library Prep Kit (Illumina, Inc., USA) according to the manufacturer's instructions. Briefly, $100 \mathrm{ng}$ total RNA from the retinas was isolated and an oligo-dT primer including an Illumina-compatible sequence at its 5' end was hybridized to the RNA and reverse transcription was carried out. After the degradation of the RNA template, second strand synthesis was initiated by a random primer containing an Illumina-compatible linker sequence at its 5' end. The double-stranded library was purified by employing AMPure magnetic beads (A63881, Beckman coulter, CA, USA) to get rid of all reaction components. The library was amplified to add the complete adapter sequences required for cluster generation. The finished library was purified from PCR components. High-throughput sequencing was carried out as paired-end 75 -sequencing reads using NextSeq 500 (Illumina, Inc., USA). Alignment of mRNA-Sequencing reads was performed using STAR-2.5.4b (Supplementary Table 10). Indices were either produced from genome assembly sequence or the representative transcript sequences for aligning to the genome and transcriptome. The alignment file was applied for assembling transcripts, estimating their abundances, and detecting differential expression of genes. Differentially expressed transcripts were determined based on counts from unique and multiple alignments using Edge $\mathrm{R}$ within $\mathrm{R}$ version 3.6.3 ( $\mathrm{R}$ development Core Team, 2011) 
using R package.

Chromatin immunoprecipitation (ChIP) sequencing. Retina tissue samples were cross-linked with 1\% paraformaldehyde for $10 \mathrm{~min}$ at $23^{\circ} \mathrm{C}$ and glycine (final concentration: $125 \mathrm{mM}$ ) was added to quench the reaction. The samples were homogenized in SDS lysis buffer (1\% SDS, $10 \mathrm{mM}$ EDTA, and $50 \mathrm{mM}$ Tris$\mathrm{HCl}, \mathrm{pH} 8.1$ ), and chromatin was then sheared using a bioruptor sonicator (Diagenode) for 40 cycles (30s on / $30 \mathrm{~s}$ off, repeated 2 times) at high power setting. After centrifugation for $10 \mathrm{~min}$ at $18,500 \mathrm{~g}$, the samples were resuspended in ChIP dilution buffer (0.01\% SDS, 1.2 mM EDTA, 1.1\% Triton X-100, $167 \mathrm{mM} \mathrm{NaCl}$, and $16.7 \mathrm{mM}$ Tris- $\mathrm{HCl}, \mathrm{pH} 8.1$ ). The sonicated lysates were subjected to immunoprecipitation using the indicated antibodies (5 $\mu \mathrm{g}$ of antibodies for each immunoprecipitation (IP) reaction) overnight. Protein A/G magnetic beads $(26162$, Thermo scientific, USA) $(30 \mu \mathrm{l})$ were added, and immunoprecipitations were continued for an additional $4 \mathrm{~h}$, and washed with low-salt wash buffer $(0.1 \%$ SDS, $1 \%$ Triton X-100, $2 \mathrm{mM}$ EDTA, $150 \mathrm{mM} \mathrm{NaCl}, 20 \mathrm{mM}$ Tris-HCl, $\mathrm{pH}$ 8.1), high-salt wash buffer (0.1\% SDS, 1\% Triton X-100, 2 mM EDTA, $500 \mathrm{mM} \mathrm{NaCl}, 20 \mathrm{mM}$ Tris-HCl, pH 8.1), LiCl immune-wash buffer (0.25M LiCl, 1\% NP40, 1\% deoxycholate, $1 \mathrm{mM}$ EDTA, $10 \mathrm{mM}$ Tris-HCl, $\mathrm{pH}$ 8.1), and Tris-EDTA buffer (1 mM EDTA, $10 \mathrm{mM}$ Tris-HCl, pH 8.0). Following the final wash, the precipitates were eluted with $100 \mu$ of IP elution buffer (1\% SDS, $0.1 \mathrm{M} \mathrm{NaHCO}_{3}$ ) twice and reverse cross-linked by incubating overnight at $65^{\circ} \mathrm{C}$ overnight. DNA/protein complexes were precipitated with $100 \%$ ethanol, air-dried, and dissolved in $20 \mu 1$ of distilled water (W4502, Sigma). The list of primers used for validation of ChIP-seq is described in Supplementary Table 11.

ChIP-seq library preparation and bioinformatic analysis. ChIP-seq library was generated by TruSeq ChIP Library Preparation Kit (Set A, IP-202-1012, Set B, IP-202-1024, Illumina, CA, USA) according to the manufacturer's instructions. Briefly, $5 \mu \mathrm{g}$ of input and ChIP-enriched DNA were end-repaired, A-tailed, adapter ligated with TruSeq index adapters, and amplified. Paired-end sequencing of all ChIP libraries was 
performed on the Illumina NextSeq 500 platform. For processing of KDM3B and histone modification ChIP-seq in the retina, all ChIP reads in FASTQ format were aligned to the GRCm 38.99 mouse genome using Bowtie2 (v2.3.4.1) and redundant reads were removed (Supplementary Table 12). Peak calling was performed with EPIC2 (v0.0.41) with the default parameters: bin size $=200$; fragment size $=150$; Gap allowed $=3$, FDR cutoff $<0.05$ ). After confirming the consistency between each replicate, we pooled extended reads to generate a bigwig track for visualization with IGV (v2.8.7).

Motif discovery. Overrepresented motif analysis of sequences enriched under KDM3B ChIP-seq peaks was performed using the de novo motif finder MEME-ChIP [http://meme.nbcr.net/meme/tools/meme-chip, date last accessed, July 2015]. A 300-bp sequence surrounding each of the ChIP-Seq peak summits (extending $150 \mathrm{bp}$ on each side) was supplied to MEME-ChIP as input and analyzed with the default settings for motif width and significance thresholds.

GO and GSEA analysis. DAVID (http://david.abcc.ncifcrf.gov) allowed significant DEGs and ChIP-seq peaks in respective gene sets to be clustered into functional gene ontologies. Enriched gene ontology terms were identified using Metascape, and scatter plots of ontology terms were made using REVIGO ${ }^{51}$. GSEA analysis was performed using (GSEA 4.1.0; http://www.broadinstitute.org/gsea/index.jsp) the MsigDB v7.1 mouse database.

Epigenomic network construction. KDM3B-centered network was constructed by integrating RNAsequencing and ChIP-Seq data analysis as follows: First, the nearest neighbor (NN) genes for KDM3B were selected by filtering condition (ChIP-count $>10$ per each gene). Next, $2^{\text {nd }} \mathrm{NN}$ genes of KDM3B were identified using NetworkAnalyst ${ }^{52}$. The selected $2^{\text {nd }} \mathrm{NN}$ gene was linked with the $1^{\text {st }} \mathrm{NN}$ gene. All networks were considered by the $3^{\text {rd }} \mathrm{NN}$. For all candidate genes of a network, genes from RNA-sequencing were filtered using FPKM $>10$. For measurement of network robustness, we compared the impact of random 
link removal on the KDM3B-centered networks between $K d m 3 b^{+/+}\left(1.5,3,6\right.$, and 12 months) and $K d m 3 b^{+/-}$ (12 months) mice.

Measurement of network structural dissimilarities. Network dissimilarity measurement was done following the approach proposed by Shieber et al. ${ }^{24}$. This method compared network structures based on quantifying differences among distance distributions, node dispersion metric, and $\alpha$-centrality.

$$
D\left(G, G^{\prime}\right)=w_{1} \sqrt{\frac{I\left(\mu_{G}, \mu_{G^{\prime}}\right)}{\log 2}}+w_{2}\left|\sqrt{N N D(G)}-\sqrt{N N D\left(G^{\prime}\right)}\right|+\frac{w_{3}}{2}\left(\sqrt{\frac{J\left(P_{\alpha G}, P_{\alpha G^{\prime}}\right)}{\log 2}}+\sqrt{\frac{J\left(P_{\left.\alpha G^{c}, P_{\alpha G^{\prime}}\right)}\right)}{\log 2}}\right)
$$

Here, $J$ represents Jensen-Shannon divergence. The first term represents dissimilarity in average node connectivity $\mu_{G}$ is the graphs averaged node-distance distribution. The second term shows dissimilarity in a node dispersion metric $(N N D(G))$. And the last term denotes the difference for distribution of $\alpha$ centrality $\left(P_{\alpha G}, P_{\alpha G^{c^{\prime}}}\right)$ that measures the influence of a node in the given graph $G$ and its complement graph $\left(G^{c}\right)$. We measured network dissimilarity following the algorithm ${ }^{24}$, using $w_{1}=0.45, w_{2}=0.45, w_{3}=$ 0.1 values.

Image analysis. The number of nuclei in ONL was counted on the middle of the $K d m 3 b^{+/+}$and $K d m 3 b^{+/-}$ mice retina. The thickness of ONL was measured using the 'length measurement' plugin of ImageJ software. The number of cone photoreceptors was counted in the middle of $K d m 3 b^{+/}$mouse inferior retina. Size of cone photoreceptor synapse was measured by using the 'area measurement' plugin of ImageJ software. Intensities of GFAP and cytochrome c were measured using the 'histogram' plugin of ImageJ software. The 3D reconstruction image was generated using Oxford Bitplane Imaris (v9.3.1, Bitplane, CT, USA)

Statistical analysis. Student's $t$-test was used to examine the significance of differences between data with GraphPad PRISM. The results are denoted as mean \pm standard error of the mean (SEM), which are obtained 
673 from two or three separated experiments. $P$ values less than 0.05 were considered statistically significant.

674

675 Data availability. RNA-sequencing and ChIP-seq data that support the findings of this study have been 676 deposited in NCBI GenBank with the primary accession code GSE160793. All other data are present in the 677 article and its Supplementary files, or are available from the corresponding author upon reasonable request. 678 Source data are provided with this paper. Recheck and GEO confirm. 


\section{REFERENCES}

680 1. Levin, O., Fujiyama, H., Boisgontier, M.P., Swinnen, S.P. \& Summers, J.J. Aging and motor inhibition: a converging perspective provided by brain stimulation and imaging approaches. Neurosci Biobehav Rev 43, 100-17 (2014).

683

2. Campello, L. et al. Aging of the Retina: Molecular and Metabolic Turbulences and Potential Interventions. Annu Rev Vis Sci 7, 633-664 (2021).

685

3. Saul, D. \& Kosinsky, R.L. Epigenetics of Aging and Aging-Associated Diseases. Int J Mol Sci 22(2021).

4. Nicetto, D. \& Zaret, K.S. Role of H3K9me3 heterochromatin in cell identity establishment and maintenance. Curr Opin Genet Dev 55, 1-10 (2019).

5. Keenan, C.R. et al. Extreme disruption of heterochromatin is required for accelerated hematopoietic aging. Blood 135, 2049-2058 (2020).

6. Ito, T., Teo, Y.V., Evans, S.A., Neretti, N. \& Sedivy, J.M. Regulation of Cellular Senescence by Polycomb Chromatin Modifiers through Distinct DNA Damage- and Histone MethylationDependent Pathways. Cell Rep 22, 3480-3492 (2018).

7. Corso-Diaz, X., Jaeger, C., Chaitankar, V. \& Swaroop, A. Epigenetic control of gene regulation

8. Mosammaparast, N. \& Shi, Y. Reversal of histone methylation: biochemical and molecular mechanisms of histone demethylases. Annu Rev Biochem 79, 155-79 (2010).

9. Kim, J.Y. et al. KDM3B is the H3K9 demethylase involved in transcriptional activation of $1 \mathrm{mo} 2$ in leukemia. Mol Cell Biol 32, 2917-33 (2012).

10. Yamane, K. et al. JHDM2A, a JmjC-containing H3K9 demethylase, facilitates transcription

11. An, M.J. et al. Histone demethylase KDM3B regulates the transcriptional network of cell-cycle genes in hepatocarcinoma HepG2 cells. Biochem Biophys Res Commun 508, 576-582 (2019).

12. Peter, I.S. \& Davidson, E.H. Evolution of gene regulatory networks controlling body plan

13. Davidson, E.H. \& Levine, M.S. Properties of developmental gene regulatory networks. Proc Natl

14. Kim, J.W. et al. NRL-Regulated Transcriptome Dynamics of Developing Rod Photoreceptors. Cell Rep 17, 2460-2473 (2016).

15. Poleshko, A. et al. $\mathrm{H} 3 \mathrm{~K} 9 \mathrm{me} 2$ orchestrates inheritance of spatial positioning of peripheral heterochromatin through mitosis. Elife 8(2019). 
16. Zhang, T., Cooper, S. \& Brockdorff, N. The interplay of histone modifications - writers that read. EMBO Rep 16, 1467-81 (2015).

17. Kim, Y.G. et al. Kdm3b haploinsufficiency impairs the consolidation of cerebellum-dependent motor memory in mice. Mol Brain 14, 106 (2021).

18. Cepko, C.L. The roles of intrinsic and extrinsic cues and bHLH genes in the determination of retinal cell fates. Curr Opin Neurobiol 9, 37-46 (1999).

19. Abler, A.S., Chang, C.J., Ful, J., Tso, M.O. \& Lam, T.T. Photic injury triggers apoptosis of photoreceptor cells. Res Commun Mol Pathol Pharmacol 92, 177-89 (1996).

20. Baden, T., Euler, T., Weckstrom, M. \& Lagnado, L. Spikes and ribbon synapses in early vision. Trends Neurosci 36, 480-8 (2013).

21. Sharma, S., Ball, S.L. \& Peachey, N.S. Pharmacological studies of the mouse cone electroretinogram. Vis Neurosci 22, 631-6 (2005).

22. Li, J. et al. KDM3 epigenetically controls tumorigenic potentials of human colorectal cancer stem cells through Wnt/beta-catenin signalling. Nat Commun 8, 15146 (2017).

23. Shaulian, E. \& Karin, M. AP-1 as a regulator of cell life and death. Nat Cell Biol 4, E131-6 (2002).

24. Schieber, T.A. et al. Quantification of network structural dissimilarities. Nat Commun 8, 13928 (2017).

25. Dehmer, M. \& Emmert-Streib, F. Comments to "Quantification of network structural dissimilarities" published by Schieber et al. Mathematical Methods in the Applied Sciences 41, 5711-5713 (2018).

26. Barabási, A.-L. Network science, (Cambridge university press, 2016).

27. Cohen, R., Erez, K., ben-Avraham, D. \& Havlin, S. Resilience of the internet to random breakdowns. Phys Rev Lett 85, 4626-8 (2000).

28. Swaroop, A., Kim, D. \& Forrest, D. Transcriptional regulation of photoreceptor development and homeostasis in the mammalian retina. Nat Rev Neurosci 11, 563-76 (2010).

29. Iwagawa, T. \& Watanabe, S. Molecular mechanisms of H3K27me3 and H3K4me3 in retinal development. Neurosci Res 138, 43-48 (2019).

30. Katoh, K., Yamazaki, R., Onishi, A., Sanuki, R. \& Furukawa, T. G9a histone methyltransferase activity in retinal progenitors is essential for proper differentiation and survival of mouse retinal cells. J Neurosci 32, 17658-70 (2012).

31. Iida, A. et al. Histone demethylase Jmjd3 is required for the development of subsets of retinal bipolar cells. Proc Natl Acad Sci U S A 111, 3751-6 (2014).

32. Hao, H. et al. Transcriptional regulation of rod photoreceptor homeostasis revealed by in vivo NRL targetome analysis. PLoS Genet 8, e1002649 (2012). 
33. Kale, J., Osterlund, E.J. \& Andrews, D.W. BCL-2 family proteins: changing partners in the dance towards death. Cell Death Differ 25, 65-80 (2018).

34. Bai, J. \& Cederbaum, A.I. Mitochondrial catalase and oxidative injury. Biol Signals Recept 10, 18999 (2001).

35. Sobrin, L. \& Seddon, J.M. Nature and nurture- genes and environment- predict onset and progression of macular degeneration. Prog Retin Eye Res 40, 1-15 (2014).

36. Smith, W. et al. Risk factors for age-related macular degeneration: Pooled findings from three continents. Ophthalmology 108, 697-704 (2001).

37. Gnana-Prakasam, J.P. et al. Loss of Hfe leads to progression of tumor phenotype in primary retinal pigment epithelial cells. Invest Ophthalmol Vis Sci 54, 63-71 (2013).

38. Diets, I.J. et al. De Novo and Inherited Pathogenic Variants in KDM3B Cause Intellectual Disability, Short Stature, and Facial Dysmorphism. Am J Hum Genet 104, 758-766 (2019).

39. Lin, L., Wang, W., Du, C. \& Luo, X. A cone-shaped concentrator with varying performances of concentrating. Opt Express 16, 6809-14 (2008).

40. Sharpe, L.T., Stockman, A., Jagle, H., Knau, H. \& Nathans, J. L, M and L-M hybrid cone photopigments in man: deriving lambda max from flicker photometric spectral sensitivities. Vision Res 39, 3513-25 (1999).

41. Brightman, D.S. et al. MLL1 is essential for retinal neurogenesis and horizontal inner neuron integrity. Sci Rep 8, 11902 (2018).

42. Barabino, A. et al. Loss of Bmil causes anomalies in retinal development and degeneration of cone photoreceptors. Development 143, 1571-84 (2016).

43. Curran, T. \& Morgan, J.I. Fos: an immediate-early transcription factor in neurons. J Neurobiol 26,

44. Oshitari, T. et al. The role of c-fos in cell death and regeneration of retinal ganglion cells. Invest Ophthalmol Vis Sci 43, 2442-9 (2002).

45. Krieg, A.J. et al. Regulation of the histone demethylase JMJD1A by hypoxia-inducible factor 1 alpha enhances hypoxic gene expression and tumor growth. Mol Cell Biol 30, 344-53 (2010).

46. Djeghloul, D. et al. Age-Associated Decrease of the Histone Methyltransferase SUV39H1 in HSC Perturbs Heterochromatin and B Lymphoid Differentiation. Stem Cell Reports 6, 970-984 (2016).

47. Lorbeck, M.T. et al. The histone demethylase Dmel|Kdm4A controls genes required for life span and male-specific sex determination in Drosophila. Gene 450, 8-17 (2010).

48. Buskin, A. et al. Disrupted alternative splicing for genes implicated in splicing and ciliogenesis causes PRPF31 retinitis pigmentosa. Nat Commun 9, 4234 (2018). 
49. Du, S. et al. Fructose-bisphosphate aldolase a is a potential metastasis-associated marker of lung squamous cell carcinoma and promotes lung cell tumorigenesis and migration. PLoS One 9, e85804 (2014).

50. Hoh Kam, J., Lenassi, E., Malik, T.H., Pickering, M.C. \& Jeffery, G. Complement component C3 plays a critical role in protecting the aging retina in a murine model of age-related macular degeneration. Am J Pathol 183, 480-92 (2013).

51. Zhou, Y. et al. Metascape provides a biologist-oriented resource for the analysis of systems-level datasets. Nat Commun 10, 1523 (2019).

52. Zhou, G. et al. NetworkAnalyst 3.0: a visual analytics platform for comprehensive gene expression profiling and meta-analysis. Nucleic Acids Res 47, W234-W241 (2019). 


\section{ACKNOWLEDGEMENTS}

789 The study was supported by the Korean Environment Industry \& Technology Institute (KEITI) through 790 “The Environmental Health Action Program" (Grant and Award number: 2017001360007) and "Digital 791 Infrastructure Building Project for Monitoring, Surveying and Evaluating the Environmental Health 792 Program" (Grant and Award number: 2021003330007) funded by the Korean Ministry of Environment 793 (MOE). This work was also supported by the National Research Foundation of Korea (NRF) grant funded 794 by the Korean government (MIST) (Basic Research Laboratory: NRF-2019R1A4A2001609; NRF795 2018R1D1A1B07050755). Kdm3b heterozygous mouse was kindly gifted by Prof. Sang-Beom Seo 796 (Chung-Ang University, South Korea). We also thank Prof. Yong-Seok Lee (Seoul National University, 797 Seoul, South Korea) and Prof. Sung Hyun Kim (Kyung Hee University, Seoul, South Korea) for their 798 scientific comments on the synaptic functions of neurons. 


\section{AUTHOR CONTRIBUTIONS}

800 Overall scientific conceptualization, J-WK; Methodology and investigation, M-JA, J-YK, JP, JhK, D-HK, 801 G-SS, H-ML, C-HK, MJK, and J-WK; Data analysis \& scientific comments, M-JA, J-YK, JP, JhK, D-HK, JkK, SR, S-BS, and J-WK; Statistical \& bioinformatics analysis, JP, JhK; Writing original draft, M-JA, JYK, and J-WK; Funding acquisition, J-WK; Supervision and project administration, J-WK. 


\section{Figure 1}

a

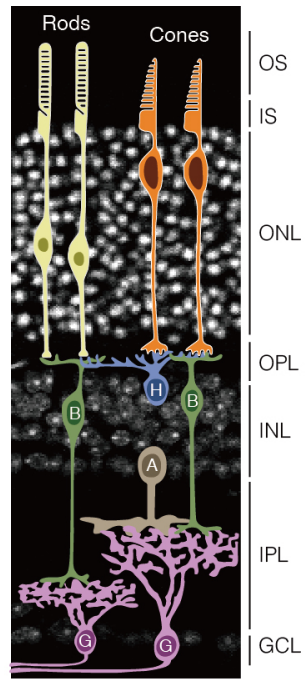

d

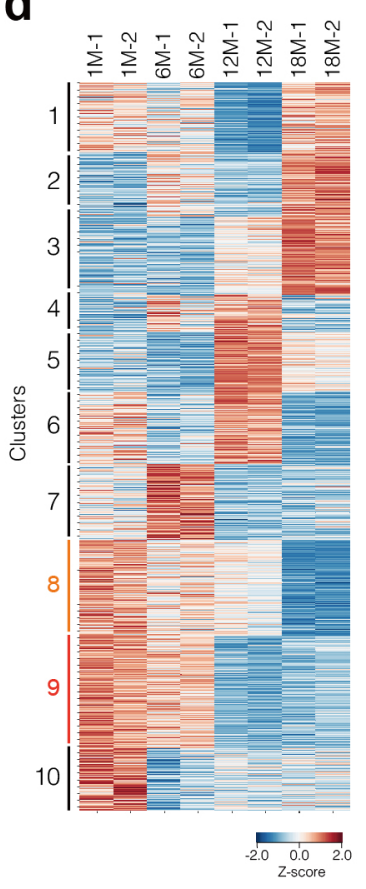

b
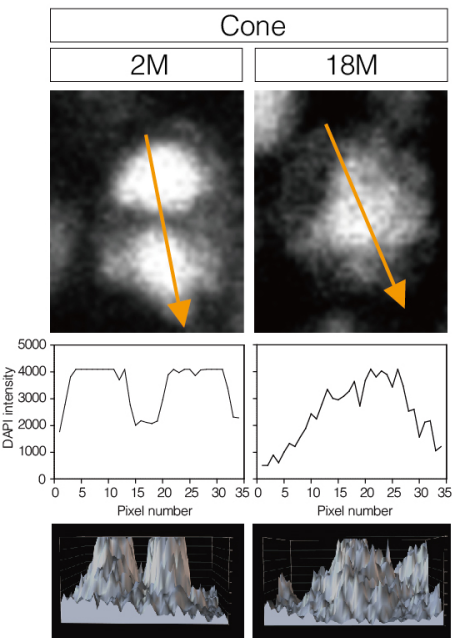

e

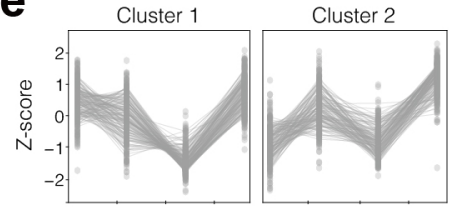

Cluster 4
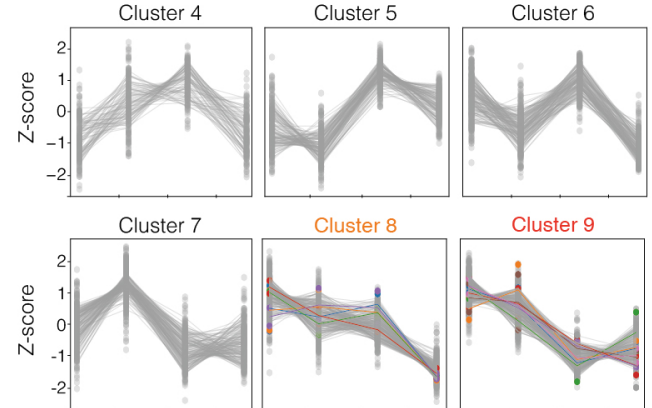

Cluster 8

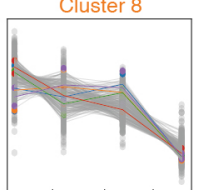

Cluster 10

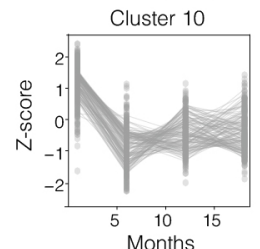

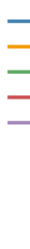

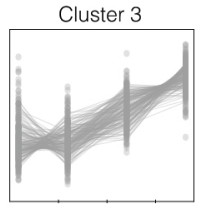

Cluster 9
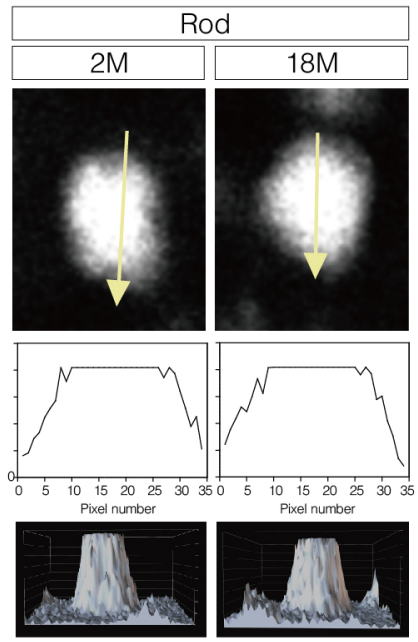

C

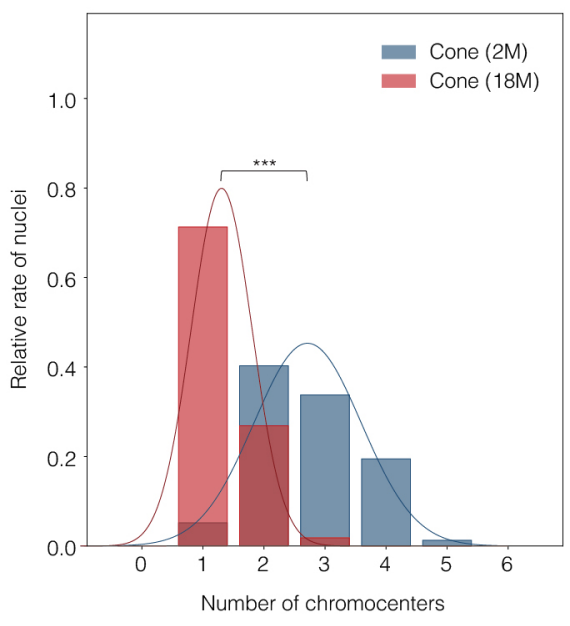

f

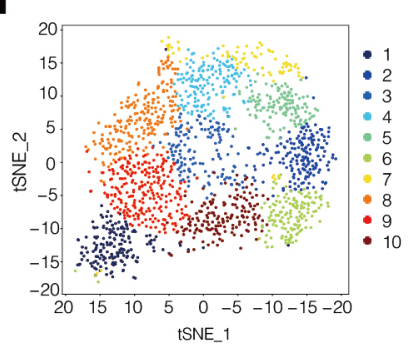

g

h
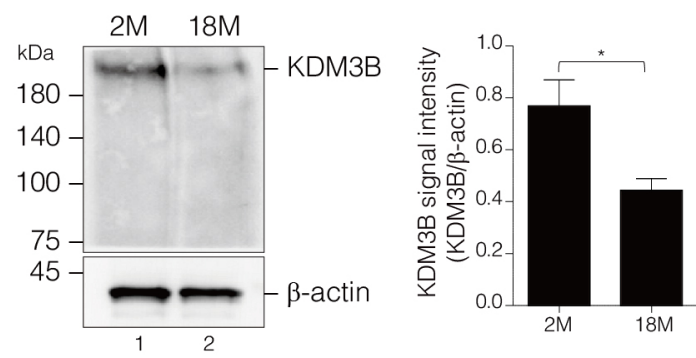

Fig. 1 Characterization of heterochromatin organization and transcriptomic profiling involved during retinal aging. a, Schematic representation of retinal structure. A; amacrine, B; bipolar. G; ganglion. H; horizontal cells. b, Representative immunofluorescence confocal images of mouse retinas stained using 4',6-diamidino-2-phenylindole (DAPI). c, Quantification of the number of chromocenters in nuclei of cones. d, Hierarchically clustered heatmap of transcripts (a total of 1,615 annotated transcripts (pre-filtered by FPKM > 30) that were differentially expressed in 1-, 6-, 12-, and 18-month-old C57BL/6J mouse retinas). e, Cluster analysis was performed by the K-means method for the gene expression profiles. f, tSNE plot revealing 10 distinct clusters identified from 1-, 6-, 12-, and 18-month-old C57BL/6J mouse retinas. g, The expression level of Kdm3b transcripts in 1-, 6-, 12-, and 18-month-old C57BL/6J mouse retinas. $\mathbf{h}$, Whole retinal lysates of 2- and 18-month-old C57BL/6J mouse were immunoblotted using anti-KDM3B antibody. $\beta$-actin was used for internal loading control. Signal intensity of KDM3B was quantified using ImageJ software. 
a

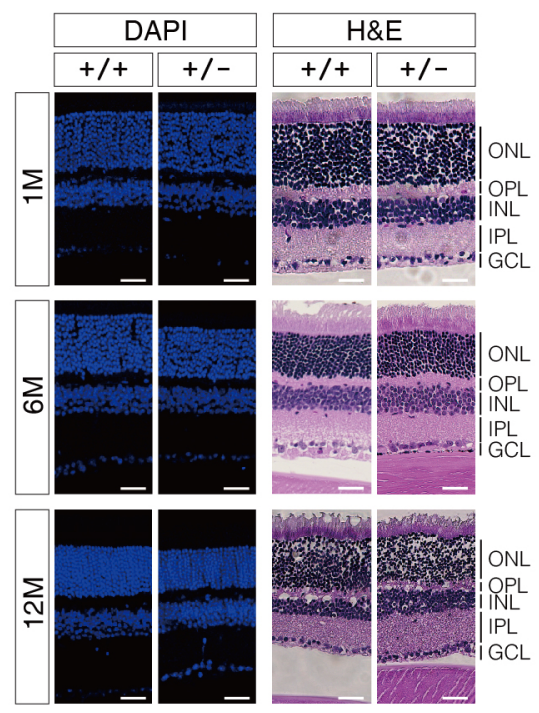

d

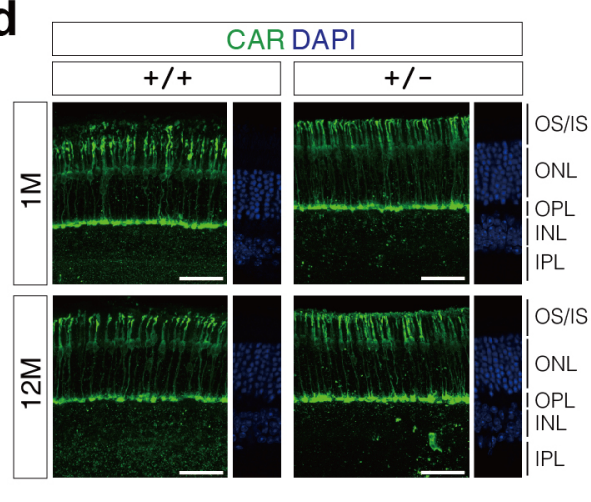

b
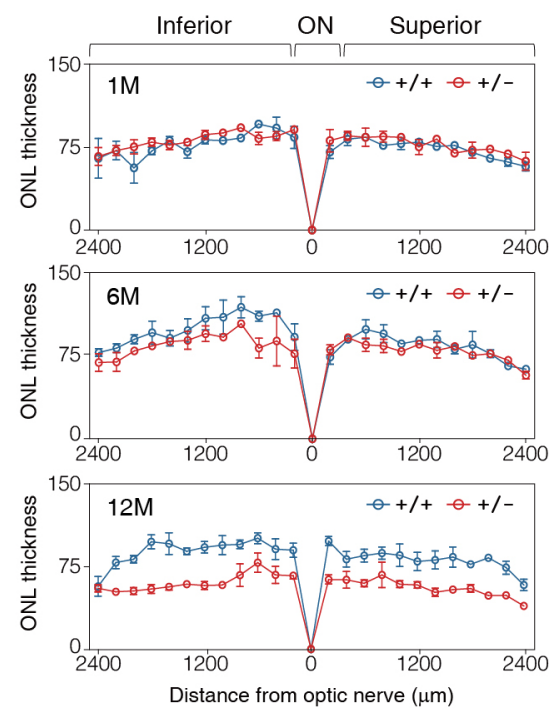

C
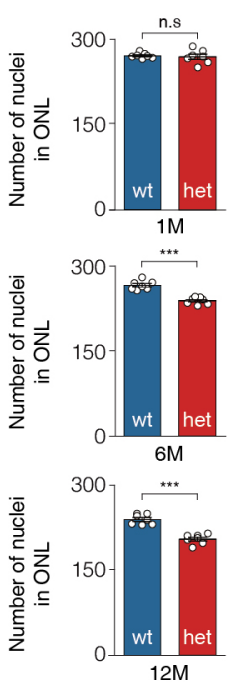

f
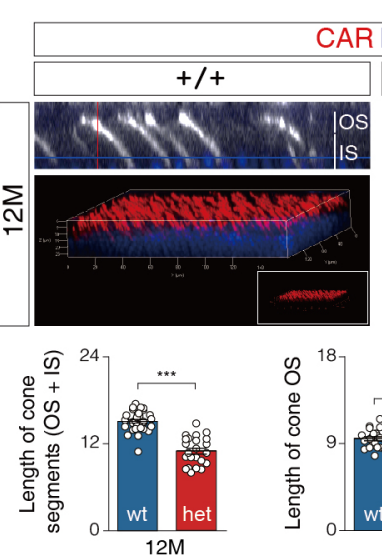

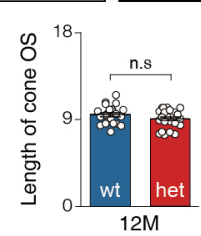

$12 \mathrm{M}$

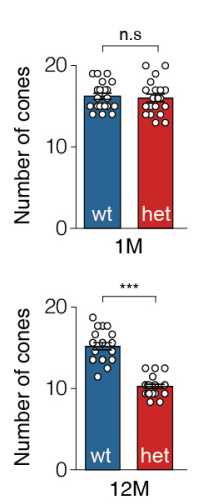

e
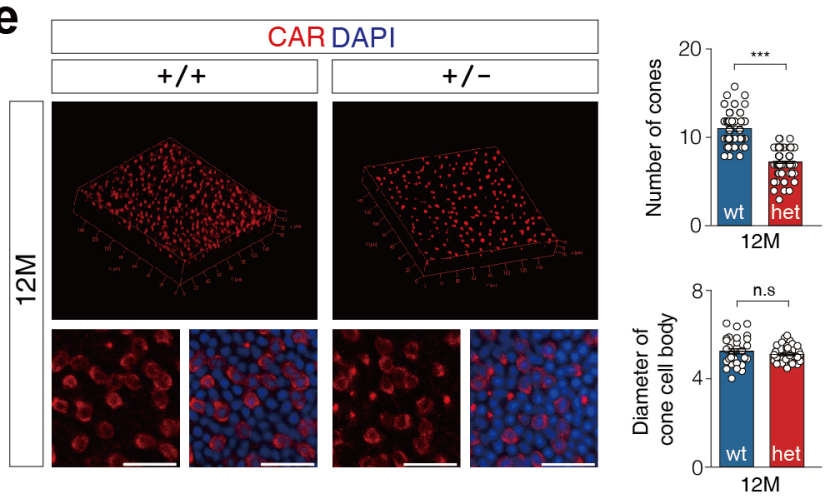

g
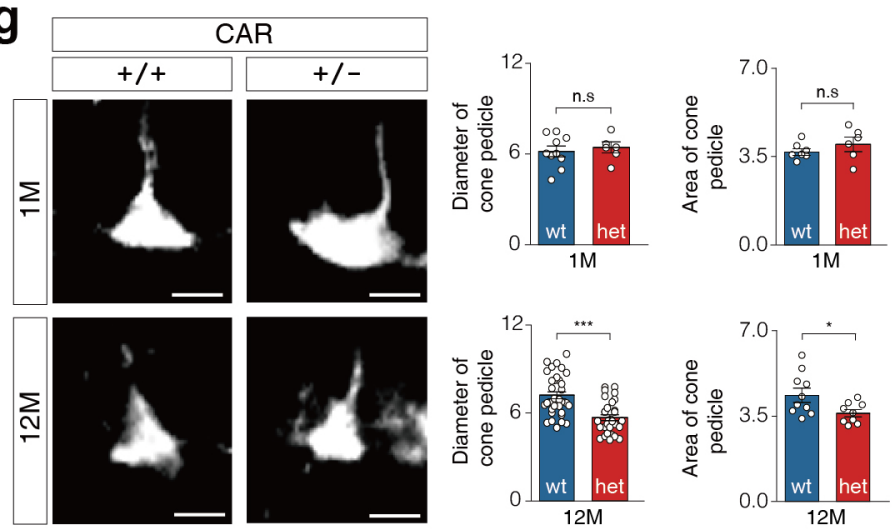

Fig. 2 The morphological changes in rods and cones in $K \boldsymbol{d} \mathbf{m} 3 \mathbf{b}^{+/}$mouse retinas. a, Cross-sectioned retinas are stained with DAPI and H\&E from 1-, 6-, and 12-month-old $K d m 3 b^{+/+}$and $K d m 3 b^{+/}$mice. Scale bar: $20 \mu \mathrm{m}$. b. Measurements of ONL thickness were made along the vertical axes of the eyes in 1, 6, and 12-month-old $K d m 3 b^{+/+}$and $K d m 3 b^{+/}$mice $(\mathrm{n} \geq 6)$. c, Quantification of the number of nuclei in ONL in $100 \mu \mathrm{m}^{2}$ of retina. d, Immunostaining of cone arrestin (CAR) in 1- and 12-month-old $K d m 3 b^{+/+}$and $K d m 3 b^{+/}$mouse retinas (left). Scale bar, $40 \mu \mathrm{m}$. The number of cones in $100 \mu \mathrm{m}^{2}$ of retina is quantified (right). e, f, Whole-mount retinas stained with CAR antibodies (red) in 12-month-old $K d m 3 b^{+/+}$and $K d m 3 b^{+/}$mice. The number of cones and diameter of the cone cell body ( $\left.\mu \mathrm{m}\right)(\mathbf{e})$, and the length of cone segments $(\mu \mathrm{m})(\mathbf{f})$ were measured. g, Cone pedicles in 12-month-old $K d m 3 b^{+/+}$and $K d m 3 b^{+/}$mouse retinas (left). Scale bar: $10 \mu \mathrm{m}$. The diameter of cone pedicles $(\mu \mathrm{m})$ and area of cone pedicles (pixel $\left.\times 10^{3}\right)$ were measured (right). Error bars show mean \pm SEM. P values obtained by Student's t-test. $* * * P<0.001$, $* P<0.05$, n.s, not significant. OS; outer segment, IS; inner segment, ONL; outer nuclear layer, OPL; outer plexiform layer, INL; inner nuclear layer, IPL; inner plexiform layer, GCL; ganglion cell layer. 
a

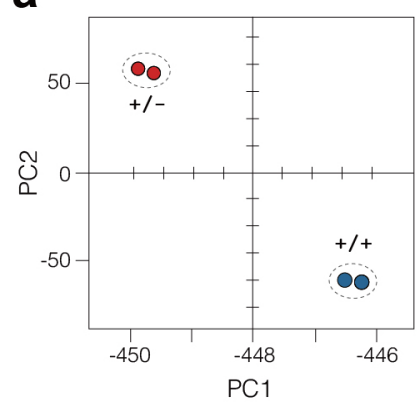

b

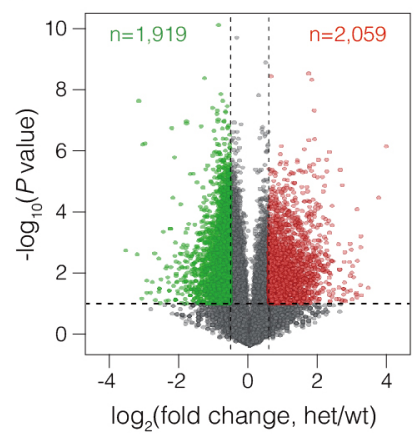

e

f

Cellular response to DNA
C
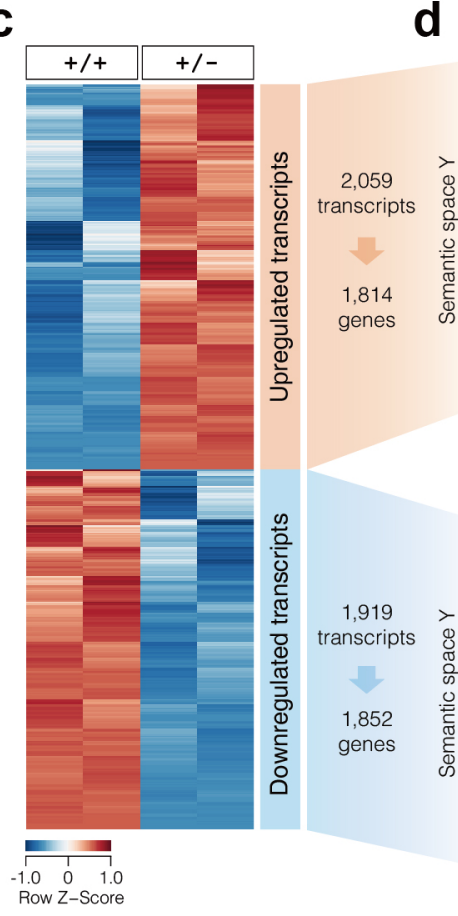

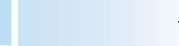

$+8$
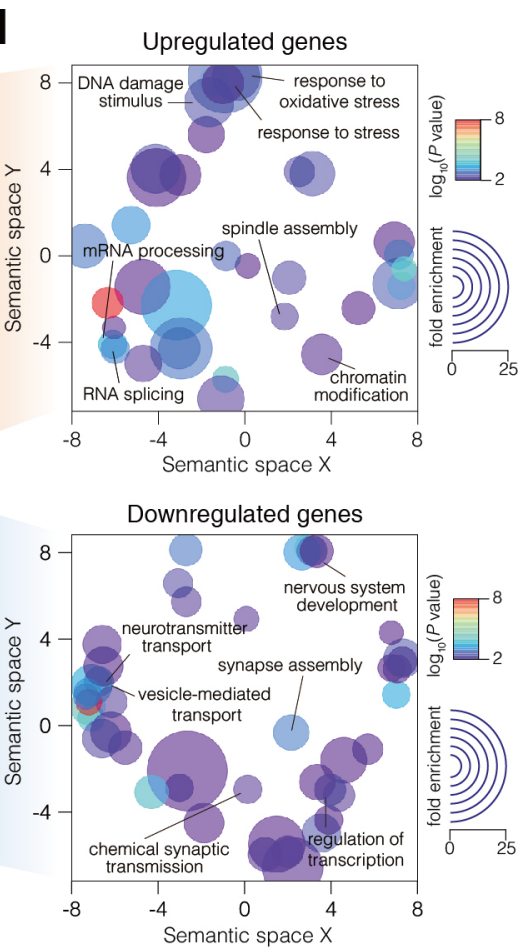
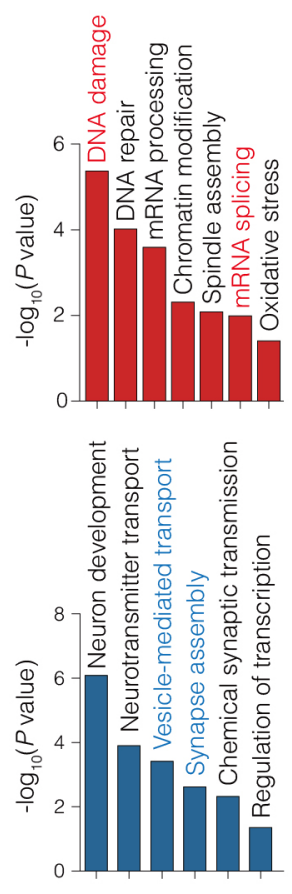
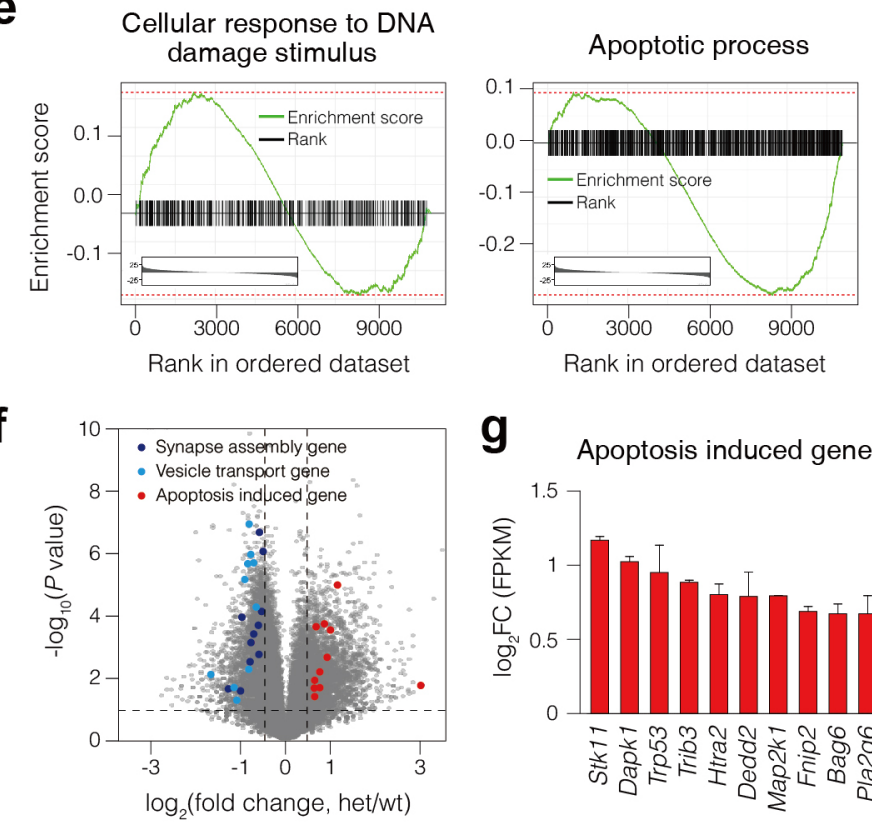

g Apoptosis induced genes

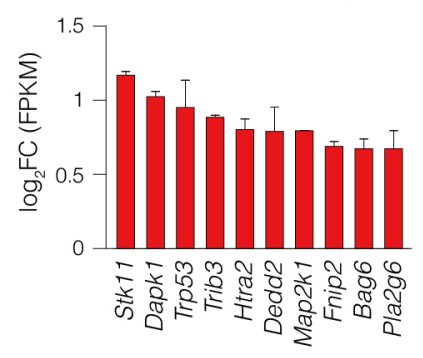

Synapse assembly

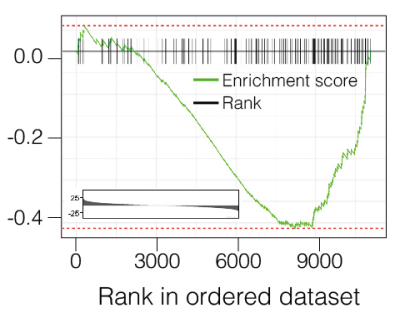

Synapse assembly genes

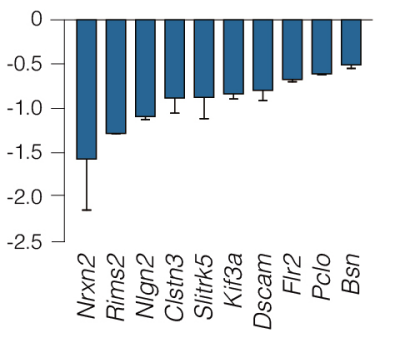

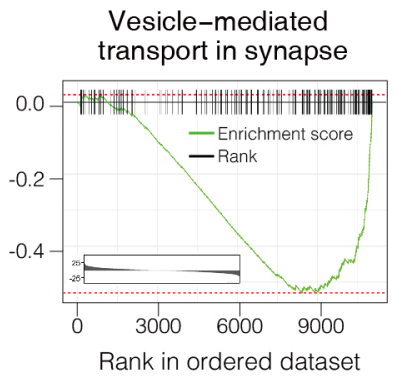

Vesicle transport genes

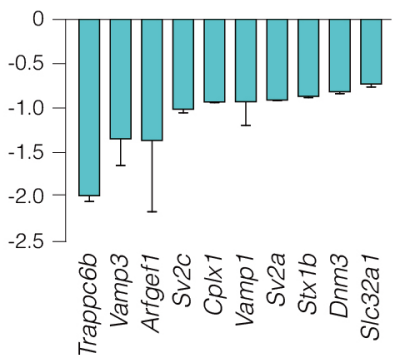

Fig. 3 Alteration of transcriptomic dynamics in $\boldsymbol{K} d \boldsymbol{m} 3 \boldsymbol{b}^{+/}$mouse retinas. a, PCA plot of RNA-sequencing from 12-month-old $K d m 3 b^{+/+}$ and $K d m 3 b^{+/}$mouse retinas. Small circles symbolize each sample, and larger circles indicate each group. $\mathbf{b}$, Volcano plot showing changes of gene expression patterns in $K d m 3 b^{+/+}$and $K d m 3 b^{+/}$mice. Expressed transcripts with strict filtering $\left(\log _{2} \mathrm{FC}>0.5, P\right.$ value $<0.05$ ) highlight only upregulated (red dots) and downregulated (green dots) transcripts. c, Hierarchical clustering of transcripts from volcano plot (a total of 3,978 transcripts) in $K d m 3 b^{+/+}$and $K d m 3 b^{+/}$mice. A clear alteration in transcriptome landscape is obvious between $K d m 3 b^{+/+}$and $K d m 3 b^{+/-}$mouse retinas. d, Scatter plot of confidence scores for enriched gene ontologies associated with differentially expressed genes, with ontologies clustered by functional similarity in the semantic space (left). Gene ontology of biological process is identified by the DAVID showing upregulated and downregulated genes in $K d m 3 b^{+-}$mouse retinas (right). e, GSEA scores for genes involved in cellular responses to DNA damage stimuli, apoptotic processes, synapse-assembly, and vesicle-mediated transport in synapses. f, Volcano plot described in (b) with three gene set categories: apoptosis-induced genes (red), synapse assembly genes (blue), and vesicle-mediated transport genes (cyan). g, Expression of genes in three categorized groups is represented in $\log _{2}$ of fold-change (FPKM). When multiple transcripts are expressed, the expression level of the most highly expressed transcript is indicated. Error bars show mean \pm SEM. 
a
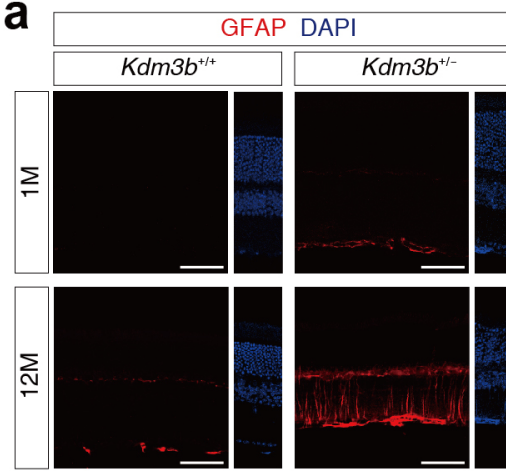
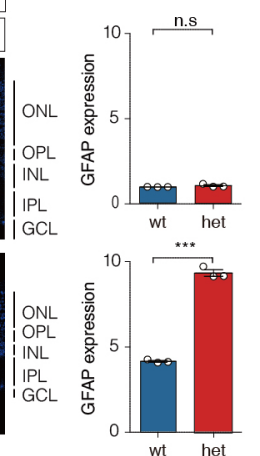

c
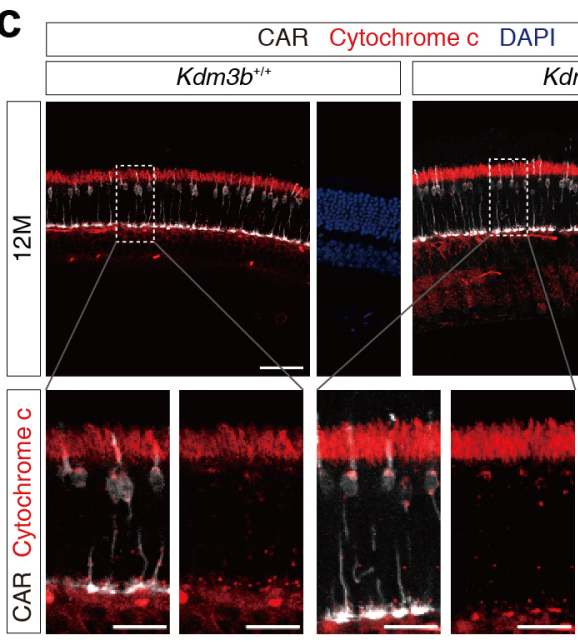

e

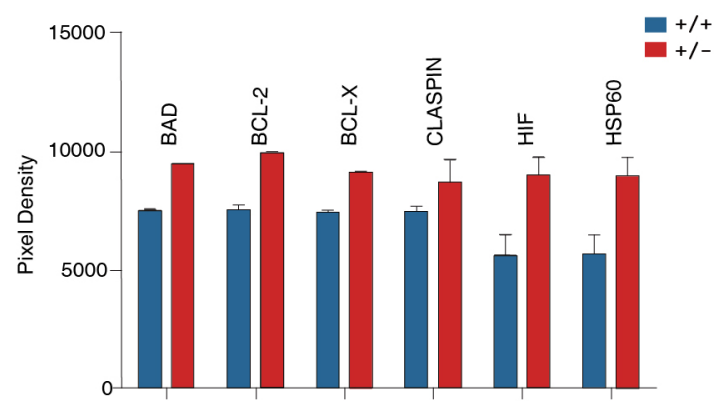

b

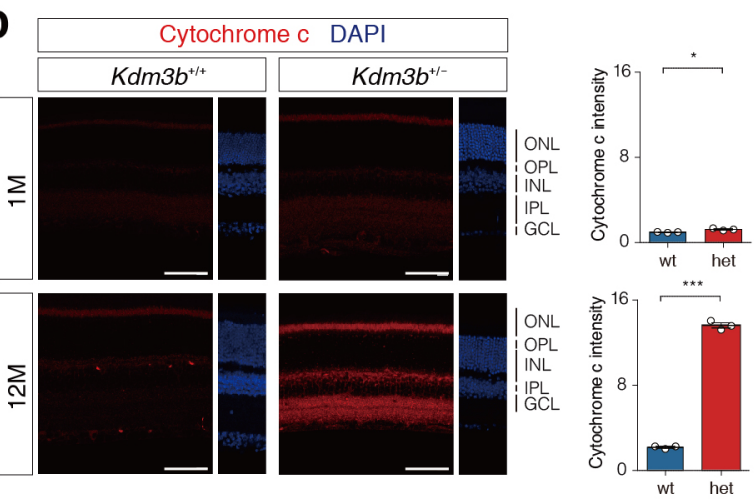

d
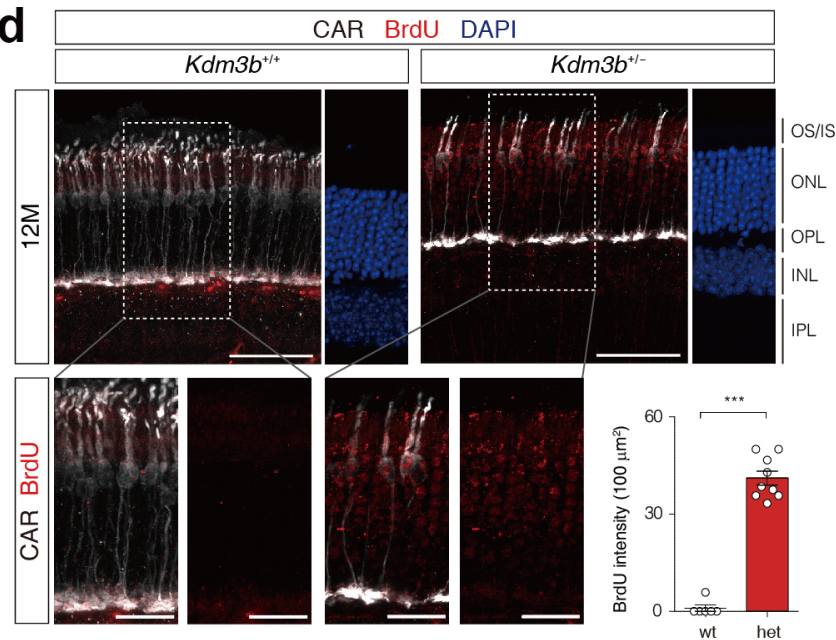

f

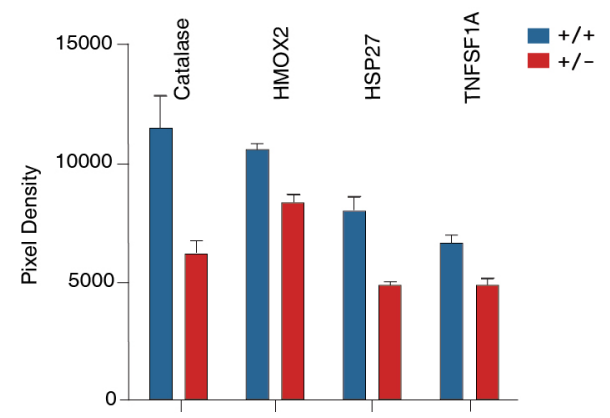

Fig. 4 Induction of apoptotic response of cones in $\mathbf{K} \boldsymbol{d} \mathbf{m} \mathbf{3} \boldsymbol{b}^{+/}$mice. a, Immunostaining of glial fibrillary acidic protein (GFAP) for the specific staining of Müller glial cells in 1- and 12-month-old $K d m 3 b^{+/+}$and $K d m 3 b^{+/}$mice (left). Scale bar: $40 \mu \mathrm{m}$. GFAP-intensity of Müller glial cells was measured using ImageJ software in 1- and 12-month-old $K d m 3 b^{+/+}$and $K d m 3 b^{+/}$retinas (right). b, Immunostaining of cytochrome c in 1- and 12-month-old $K d m 3 b^{+/+}$and $K d m 3 b^{+/}$retinas (left). Scale bar: $40 \mu \mathrm{m}$. Cytochrome c intensity of the retinas in 1and 12-month-old $K d m 3 b^{+/+}$and $K d m 3 b^{+/}$mice was measured using ImageJ (right). c, Double immunostaining of cytochrome c (red) and CAR (white) in 12-month-old $K d m 3 b^{+/+}$and $K d m 3 b^{+/}$retinas. Scale bar: $40 \mu \mathrm{m}$. High magnification images in the white box showing cytochrome c (red) expression in cones (white arrowhead). Scale bar: $20 \mu \mathrm{m}$. The signal intensity of cytochrome c-positive cones was measured. d, Apoptotic cells were detected by TUNEL assay (BrdU positive cells) in 12 -month-old $K d m 3 b^{+/+}$and $K d m 3 b^{+/}$retinas. Scale bar: $50 \mu \mathrm{m}$. High magnification images in the white box showing BrdU (red) staining in cone photoreceptors (white). Scale bar: $20 \mu \mathrm{m}$. The percentage of BrdU-positive cones. Error bars show mean \pm SEM. $P$ values obtained by Student's t-test. *** $P<0.001, * P<0.05$, n.s, not significant. e, f, Relative protein expression levels were estimated after quantifying the pixel densities in autoradiograms using ImageJ. 
a
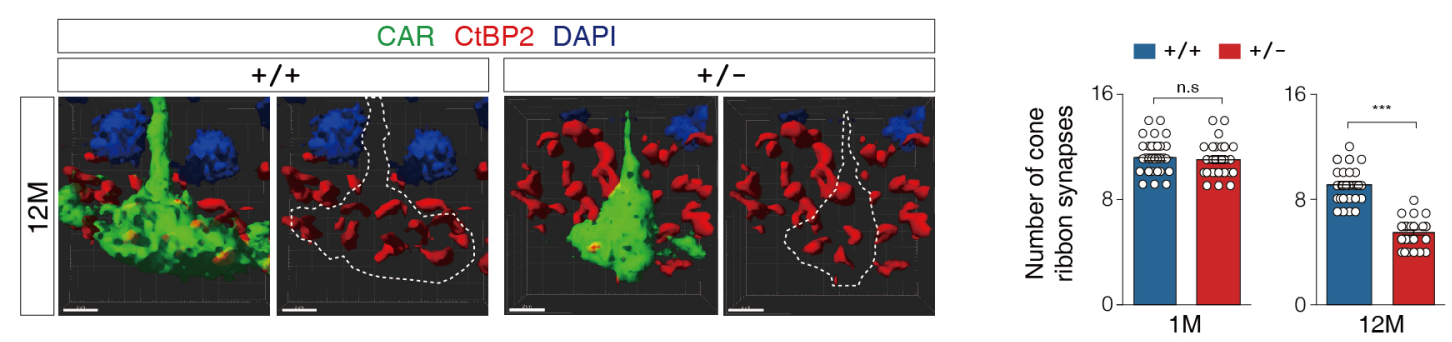

b

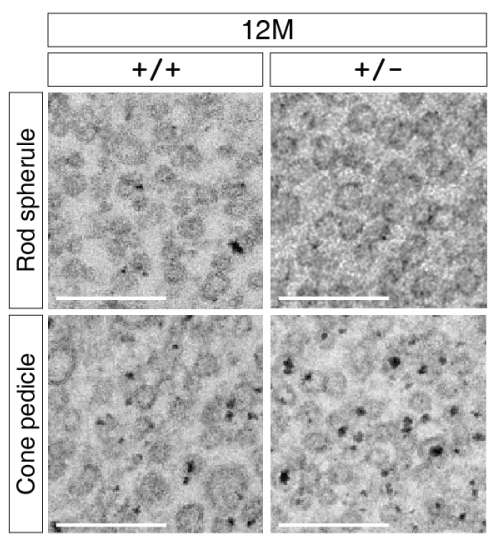

c
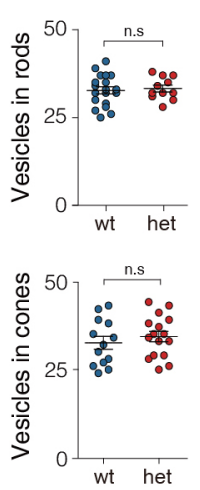
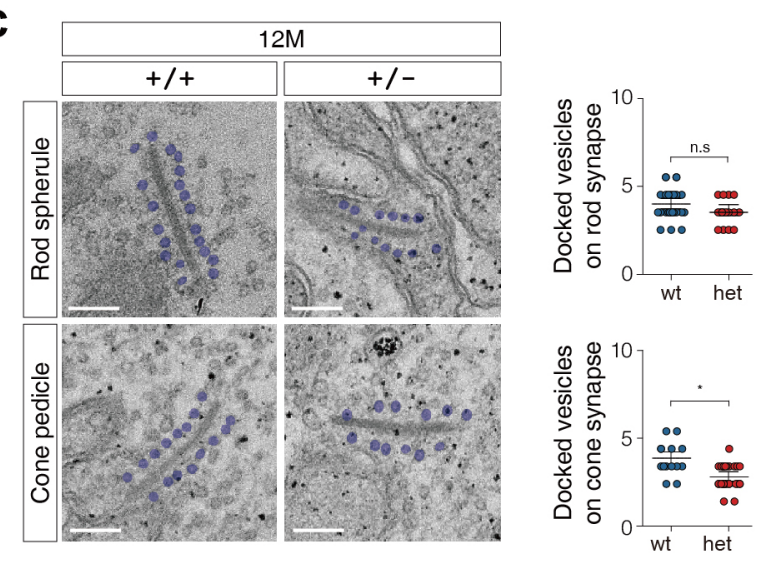

d

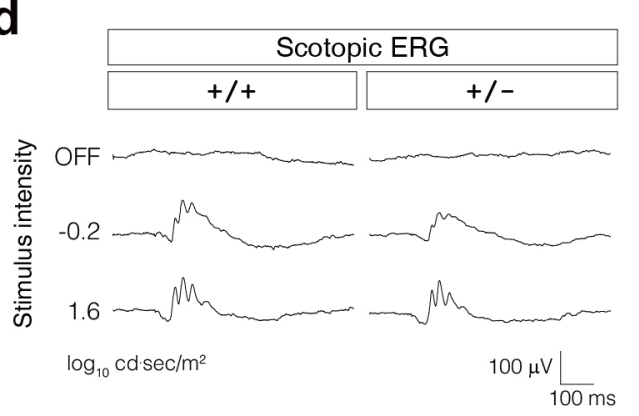

g
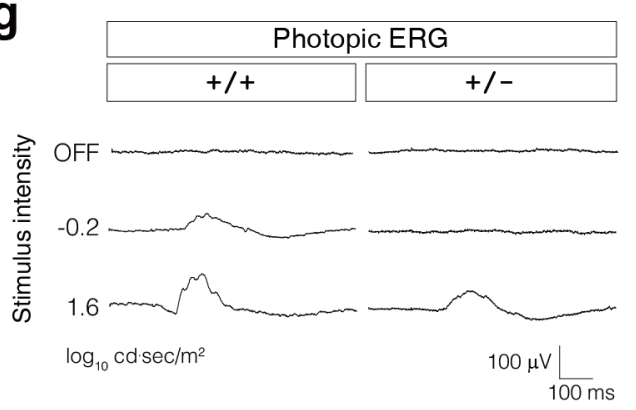
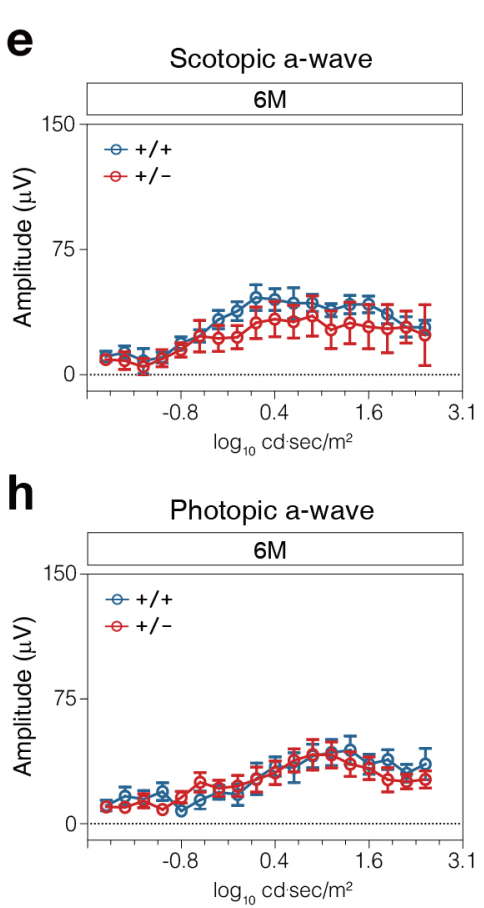
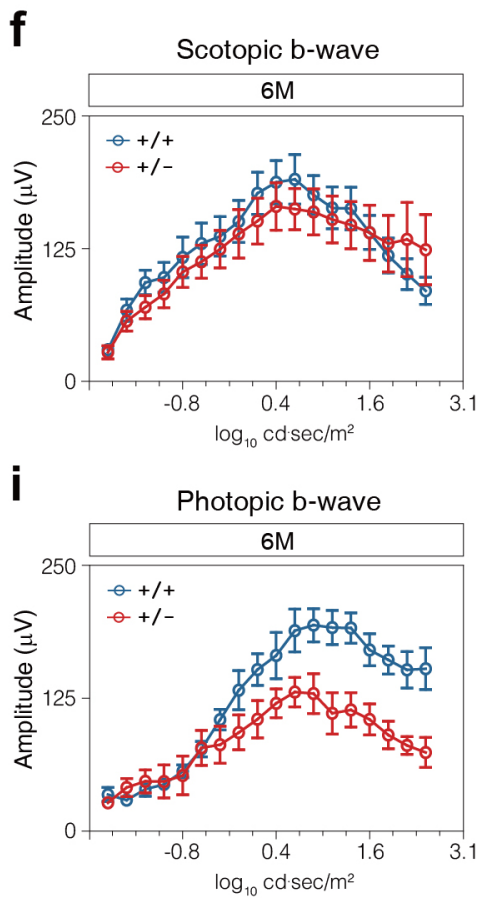

Fig. 5 The alteration of synaptic morphology of cones in $\mathbf{K d m} \mathbf{3} \boldsymbol{b}^{+/}$mice. a, 3D reconstruction images showing ribbon synapses in the cone photoreceptors of 12 -month-old $K d m 3 b^{+++}$and $K d m 3 b^{+/}$mice. Scale bar: $5 \mu \mathrm{m}$. Immunostaining with CtBP2 (C-terminal binding protein) and CAR antibodies examined in 1 and 12-month-old $K d m 3 b^{+/+}$and $K d m 3 b^{+/}$mice (left). The number of ribbon synapses is measured in a single cone pedicle (right). Scale bar: $2 \mu \mathrm{m}$. b. Transmission electron microscopy (TEM) images of the vesicles in rod spherules and cone pedicles of 12-month-old $K d m 3 b^{+/+}$and $K d m 3 b^{+/}$mice (left). The number of vesicles in a single rod spherule and cone pedicle $\left(0.16 \mu \mathrm{m}^{2}\right)$ (right). Scale bar: $0.2 \mu \mathrm{m}$. c, TEM images of docked vesicles (shaded with light cyan) near the rod and cone ribbon synapse (left). Scale bar: $0.2 \mu \mathrm{m}$. The number of docked vesicles near the rod and cone ribbon synapse in 12-month-old $K d m 3 b^{+/+}$and $K d m 3 b^{+/-}$mice $(0.1 \mu \mathrm{m}$ of each ribbon synapse) (right). Error bars show mean \pm SEM. $P$ values obtained by Student's t-test. $* P<0.05$, n.s, not significant. d-i, Electroretinograms (ERGs) of 6-month-old $K d m 3 b^{+/+}$and $K d m 3 b^{+/}$mice in 17 different stimulus flashes (from -1.7 to $3.1 \log _{10} \mathrm{~cd} \mu \mathrm{sec} / \mathrm{m}^{2}$ ) presented in darkness (scotopic) and superimposed upon a steady rod-desensitizing adapting field (photopic). d, Representative scotopic ERGs elicited by two different stimuli (OFF, -0.1, and $1.6 \log _{10}$ cd $\mu \mathrm{sec} / \mathrm{m}^{2}$ ) from 6-month-old $K d m 3 b^{+/+}$and $K d m 3 b^{+/-}$ mice. e, f, The scotopic amplitudes of a-waves (e) and b-waves (f) are shown as functions of the stimulus intensity. $\mathbf{g}$, Representative photopic ERGs elicited by two different stimuli (OFF, -0.1 , and $1.6 \log _{10} \mathrm{~cd} \mu \mathrm{sec} / \mathrm{m}^{2}$ ) from 6-month-old $K d m 3 b^{+/+}$and $K d m 3 b^{+/}-$mice. h, i, The photopic amplitudes of a-waves (h) and b-waves (i) are shown as functions of the stimulus intensity. Graphs show the mean $\pm \mathrm{SEM}, \mathrm{n}$ $=7$ per each genotype. 
a
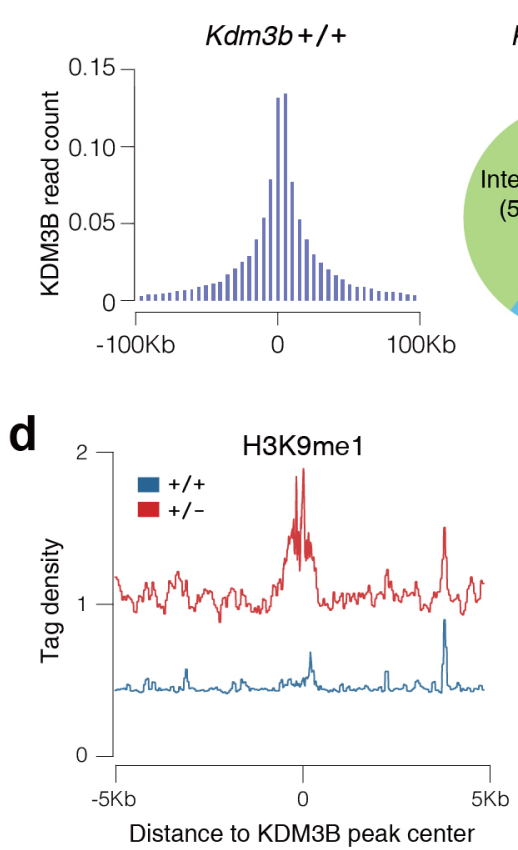

b

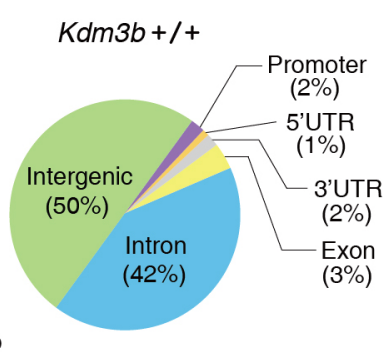

C
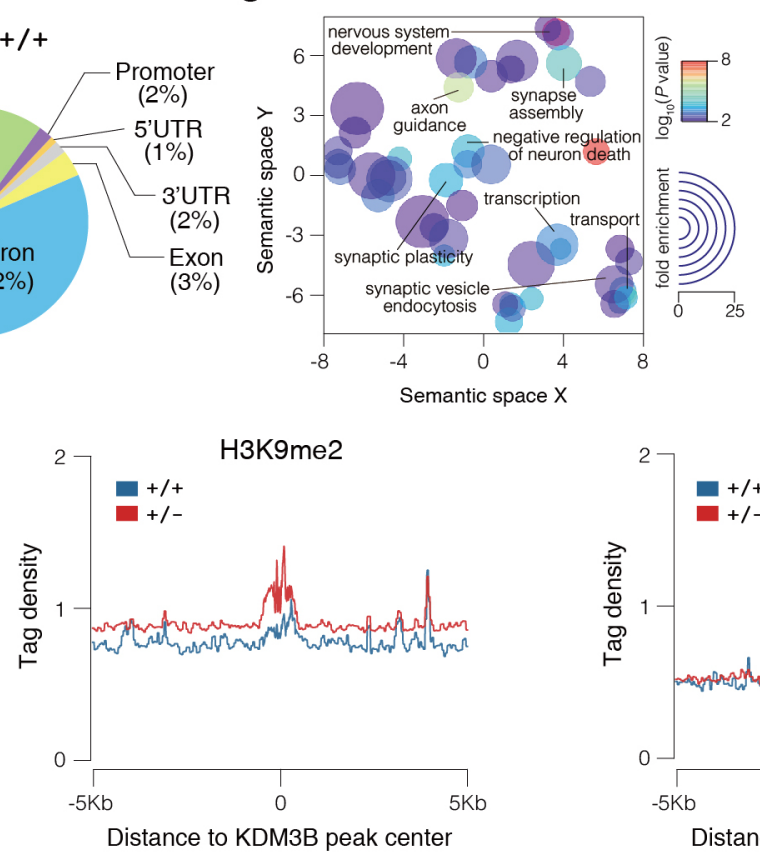

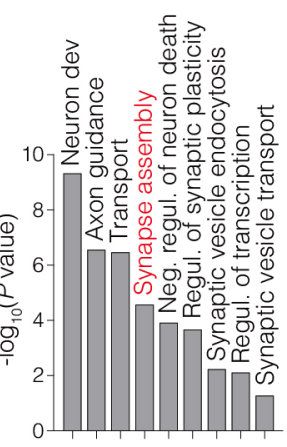

H3K9me3

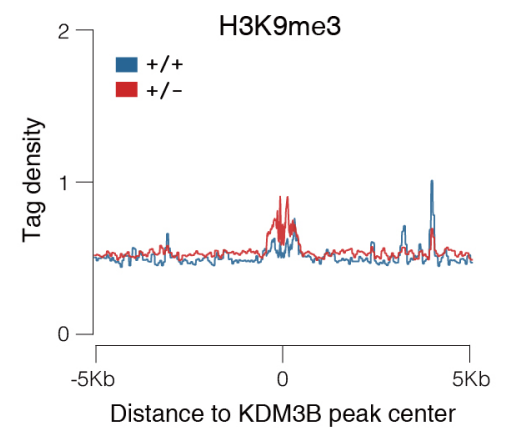

e

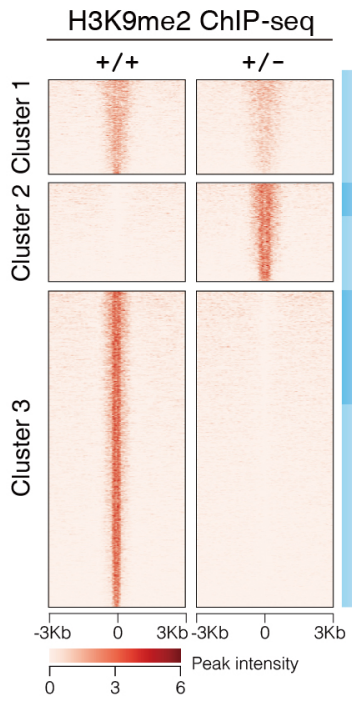

f

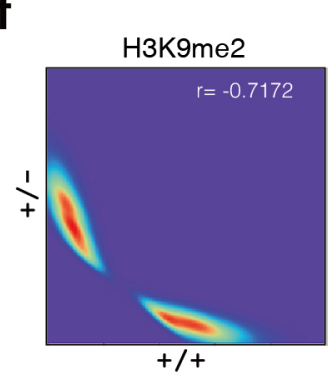

H3K9me2 binding intensity
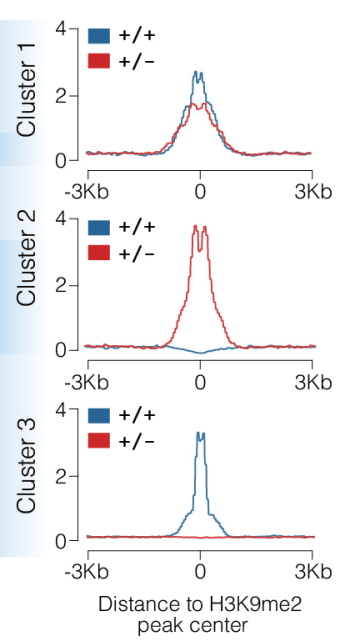

g

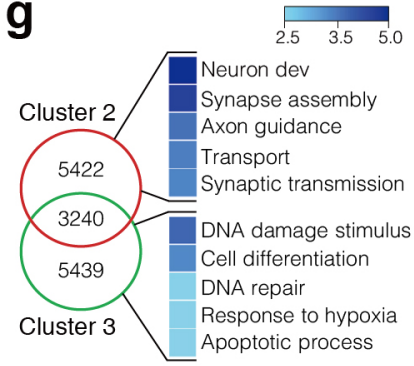

h

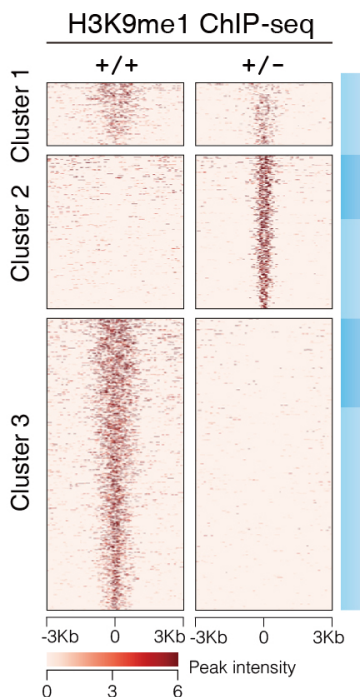

H3K9me1 binding intensity
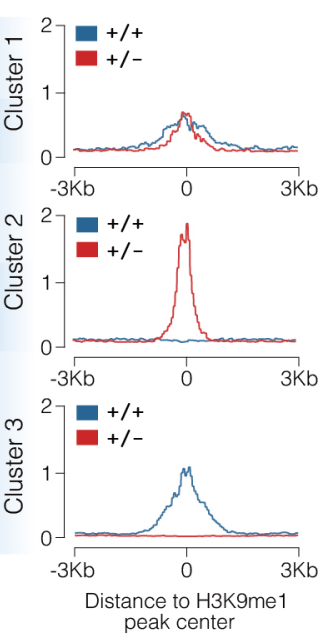

i

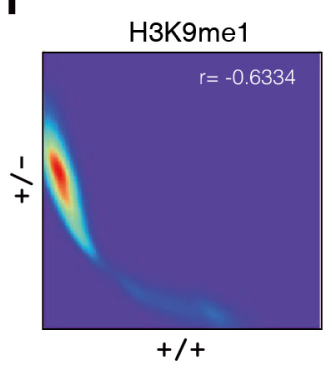

j

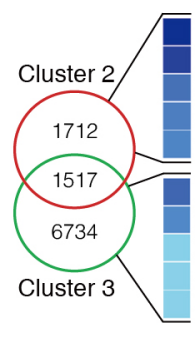

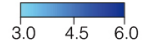

Transport

Regulation of transcription Synaptic transmission Synaptic vesicle transport Synaptic vesicle exocytosis

Metabolic process

Biological regulation Developmental process Glucose homeostasis Regulation of signaling

Fig. 6 The disruption of global $\mathbf{H 3 K 9}$ methylation in $\mathbf{K d m} 3 \mathbf{b}^{+/}$mouse retinas. a, Bar graph shows the distribution of KDM3B peaks around transcriptional start sites. b, Pie chart of KDM3B enrichment distribution at genomic loci within the genome, including promoters, exons, introns, 5'-untranslated regions (5'-UTRs), 3'-UTRs, and intergenic regions (outside -10 to $+10 \mathrm{~Kb}$ of genes) in $K d m 3 b^{+/+}$mouse retinas. c, Scatter plot of confidence scores for enriched gene ontologies associated with KDM3B ChIP-seq signal, with ontologies clustered by functional similarity in the semantic space (left). Gene ontology of biological process is identified by the DAVID showing KDM3B-enriched peaks (right). d, Plots of H3K9me1, H3K9me2, and H3K9me3 ChIP-seq signal intensity relative to the center of KDM3B occupied sites ( $\pm 5 \mathrm{~Kb})$ in $K d m 3 b^{+/+}$and $K d m 3 b^{+/}$mouse retinas. e, h, Heatmap view of H3K9me2 (e) and H3K9mel (h) ChIP-Seq-read intensity around H3K9me2 (e) and H3K9mel (h) peak center $( \pm 3 \mathrm{~Kb})$ detected in $K d m 3 b^{+/+}$and $K d m 3 b^{+-}$mouse retinas. The plot shows the mean H3K9me2 and H3K9me 1 binding intensities to the center of each peak. f, i, Scatter density plot of input-normalized H3K9me2 (f), and H3K9mel (i) RPKM between $K d m 3 b^{+/+}$and $K d m 3 b^{+/}$at $\mathrm{H} 3 \mathrm{~K} 9 \mathrm{me} 2$ peak TSS regions (f), and at H3K9mel peak TSS regions (i). $\mathbf{g}$, j, Gene ontology of biological process is identified by the DAVID showing H3K9me2 (g) and H3K9mel (j) enriched peaks in $K d m 3 b^{+/+}$and $K d m 3 b^{+/}$mouse retinas. 


\section{Figure 7}

a

Upregulated genes (RNA-seq)

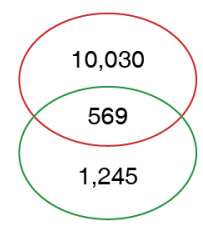

KDM3B (ChIP-seq)

b

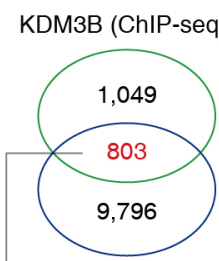

Downregulated genes (RNA-seq)
C
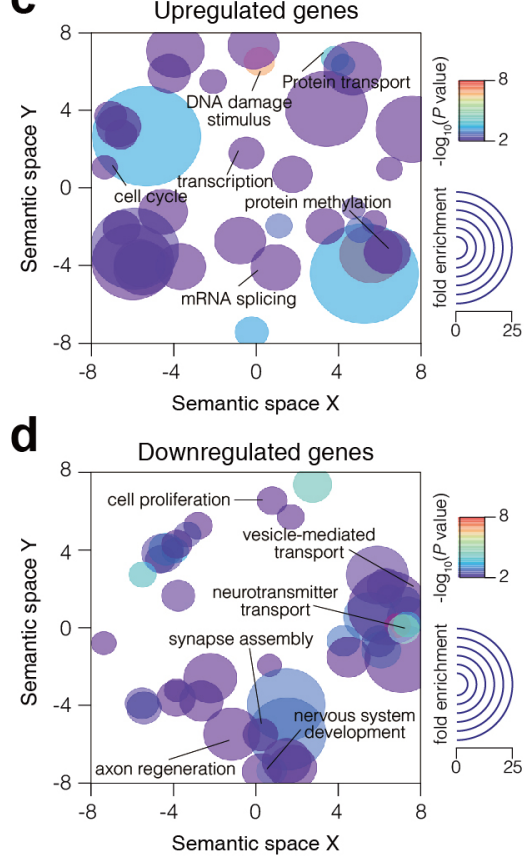
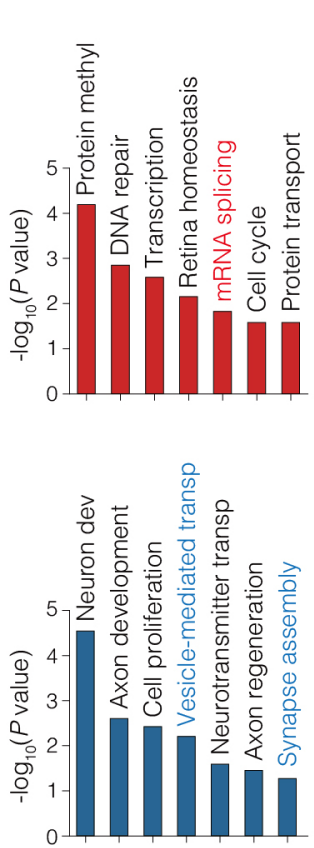

e

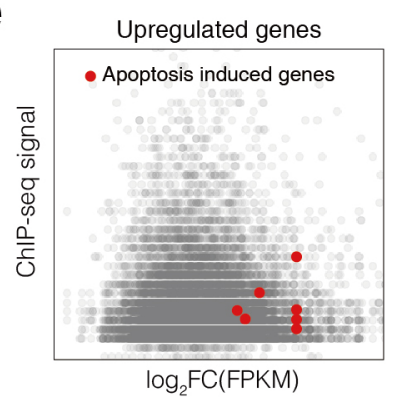

f

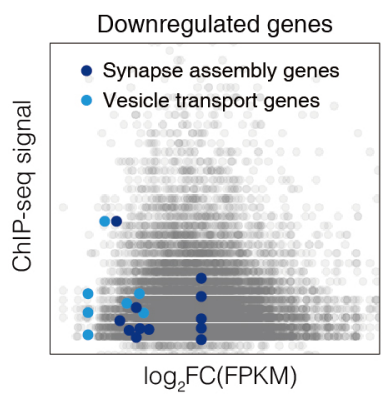

9
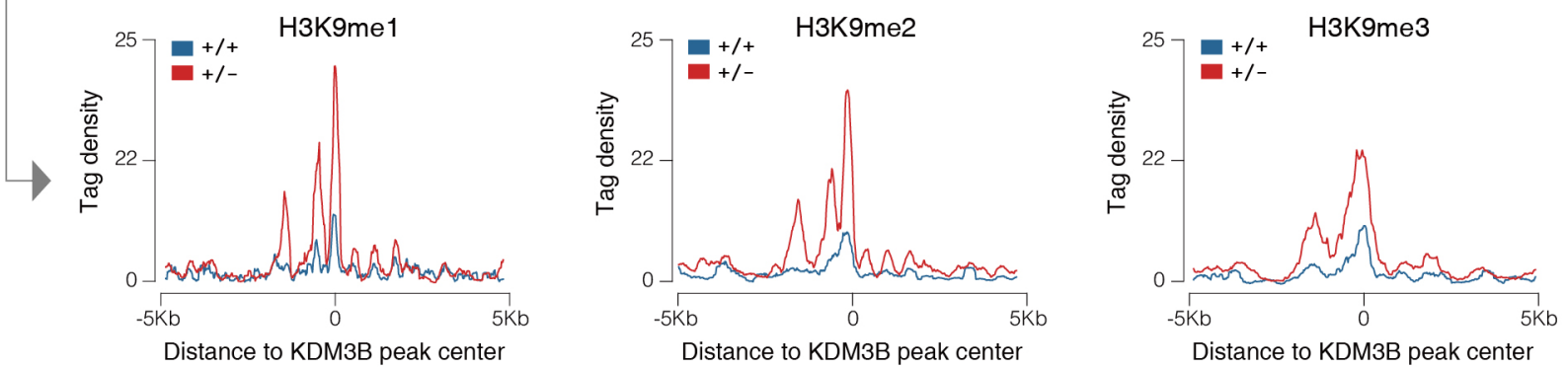

h

Nrxn2

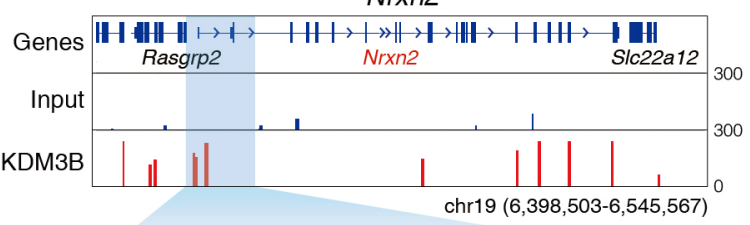

$1 \mathrm{~Kb}$
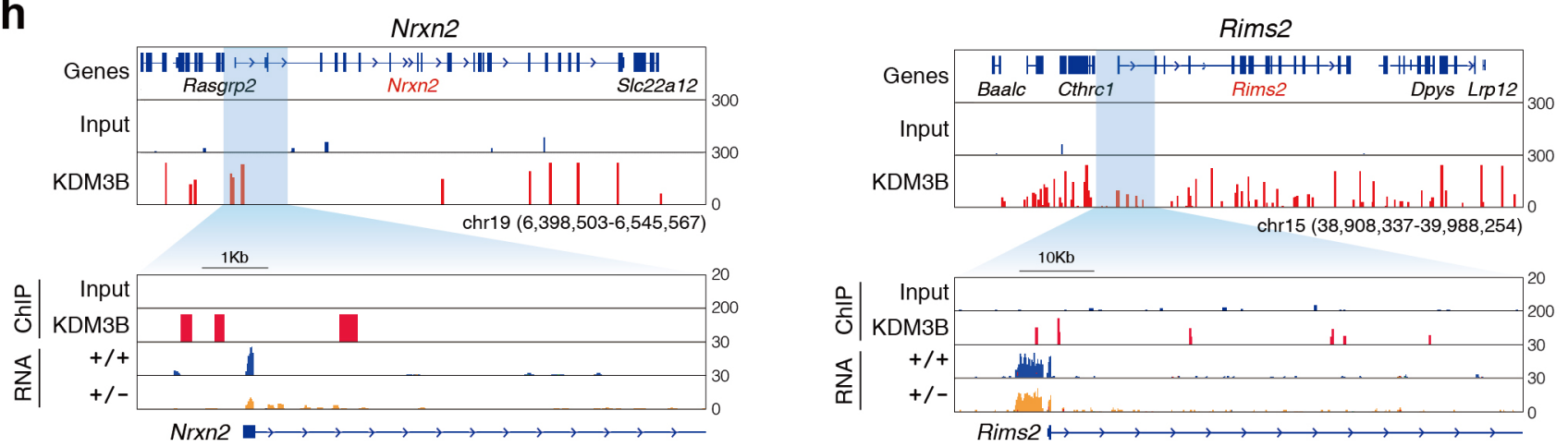

i

a +/+ a +/-
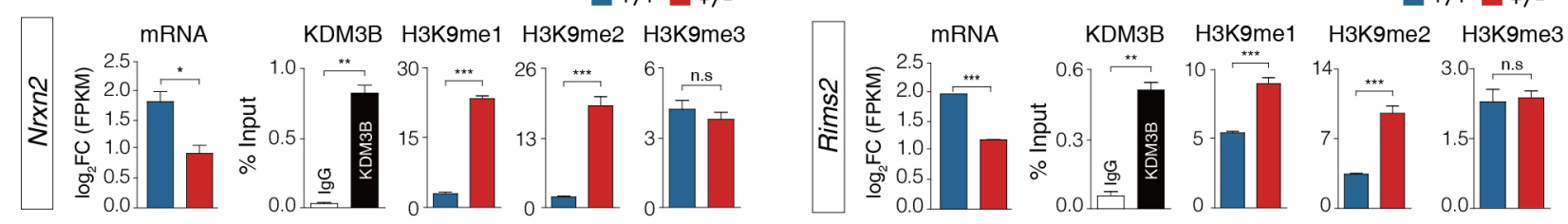

Fig. 7 The global correlation between transcriptome and epigenome dynamics during mouse retinal aging. a, b, Venn diagram showing the overlap between the genes including KDM3B peaks and upregulated (a) /or downregulated (b) KDM3B-target genes based on RNA-sequencing data. c, d, Scatter plot of confidence scores for enriched gene ontologies associated with upregulated genes (c) and downregulated genes (d) and their enrichments for KDM3B, with ontologies clustered by functional similarity in the semantic space. Gene ontology of biological process shows upregulated and downregulated genes integrated with RNA-sequencing and ChIP-seq analysis. e, f, Scatter plot showing changes in expression of upregulated (e) or downregulated genes (f) in $K d m 3 b^{+++}$and $K d m 3 b^{+/}$mouse retinas and their enrichments for KDM3B. g, Plots of H3K9me1, H3K9me2, and H3K9me3 ChIP-seq signal intensity relative to the center of downregulated KDM3B occupied sites $( \pm 5 \mathrm{~Kb})$ in $K d m 3 b^{+/+}$and $K d m 3 b^{+/-}$mouse retinas. h, ChIP-seq tracks of KDM3B in $K d m 3 b^{+/+}$along the Nrxn2 and Rims 2 locus. Selected genomic elements (shaded with light cyan) indicate KDM3B ChIP-seq peaks at Nrxn2 and Rims 2 promoter regions. i, Recruitment and presence of KDM3B, H3K9mel, H3K9me2, and H3K9me3 at Nrxn2 and Rims 2 promoter regions are validated by ChIP-qPCR. Results are presented as the mean \pm SEM (error bars). $P$ values obtained by Student's t-test. *** $P<0.001, * * P<0.01$, n.s, not significant. 
a

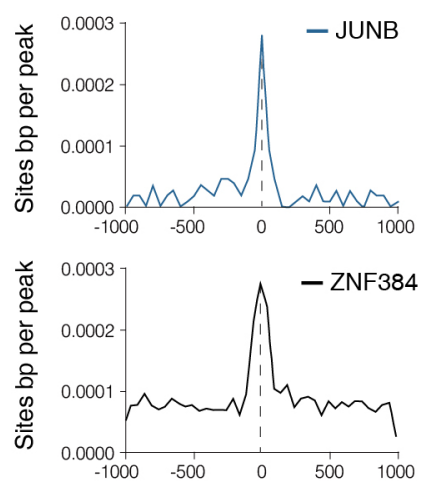

b

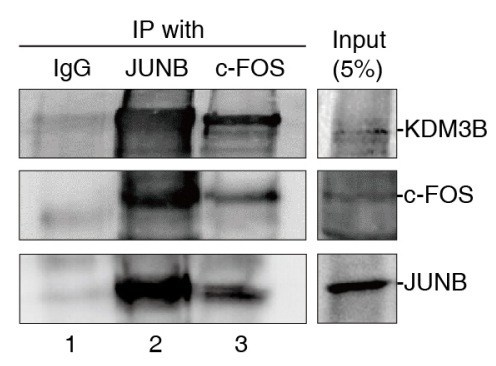

C

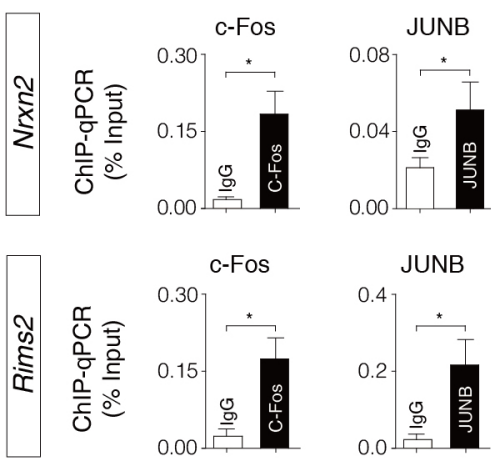

d

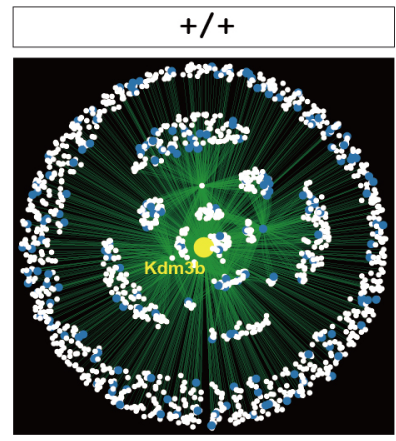

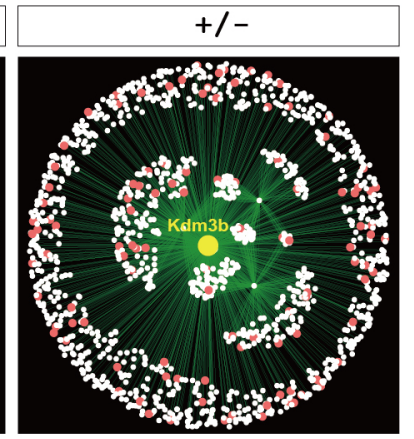

f

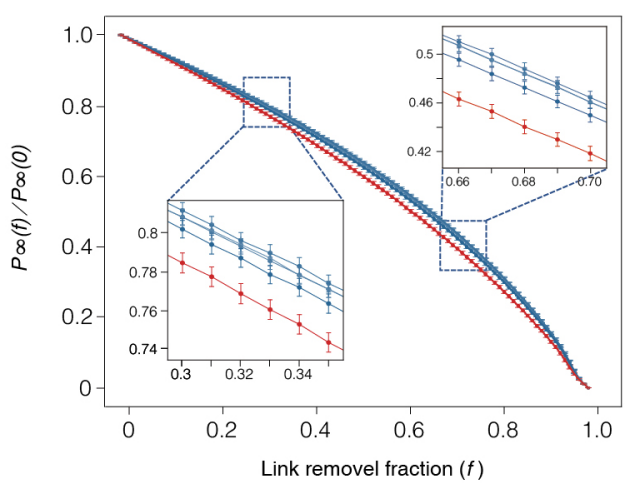

$+/+1.5 \mathrm{M}$

$+/+3 \mathrm{M}$

$+/+6 \mathrm{M}$

$+/+12 \mathrm{M}$

$+/-12 \mathrm{M}$ e

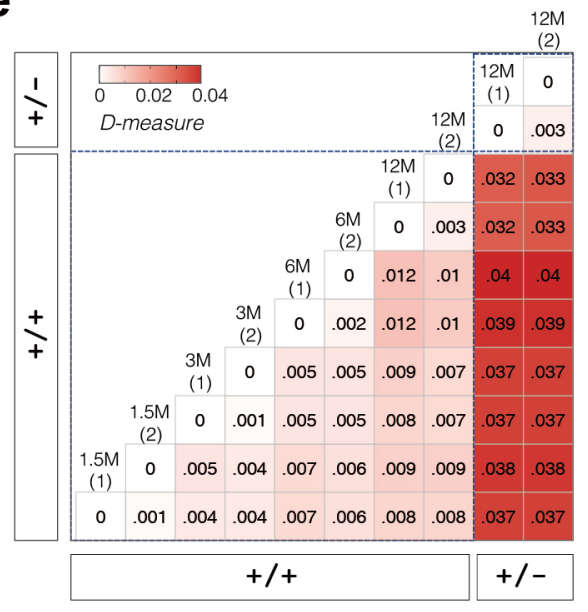

g

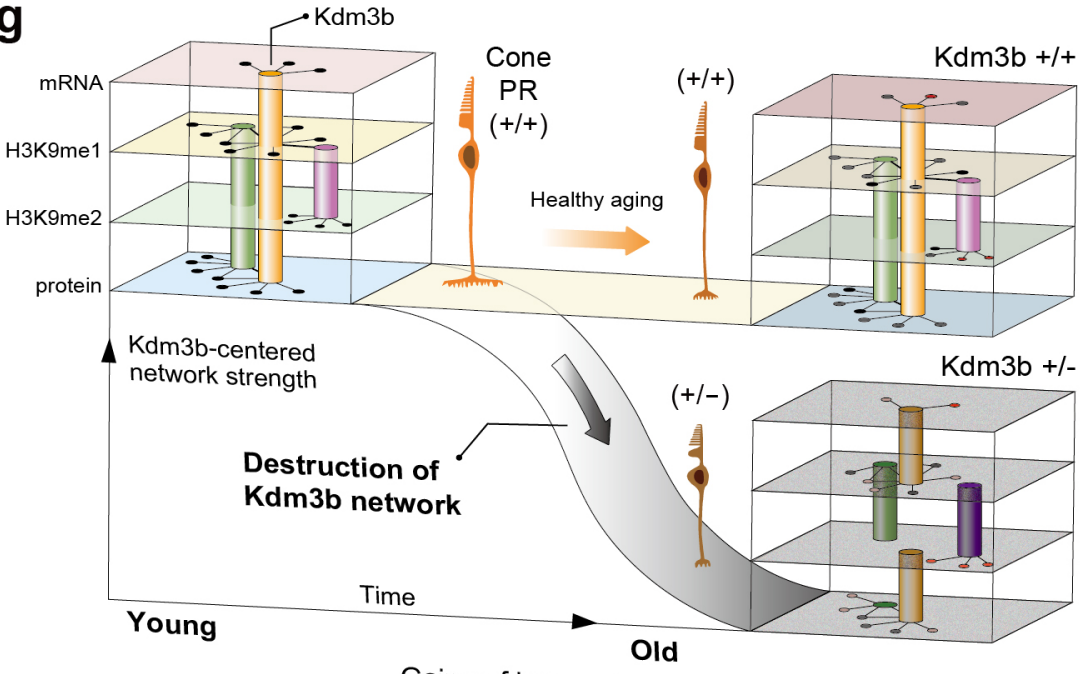

Gains of heterochromatin: pre-synaptic function Losses of heterochromatin: apoptotic function

Fig. 8 The measurement of structural difference and robustness of KDM3B-centered networks. a, The graph represents the distribution of occurrence probabilities of the consensus sequences enriched under KDM3B ChIP-Seq peaks with respect to ZNF384 and JUN motifs. b, Immunoblot showing co-immunoprecipitation of endogenous KDM3B with c-Fos and JUNB in mice retinas. c, Recruitment and presence of c-Fos and JUNB at Nrxn2 and Rims 2 promoter regions are validated by ChIP-qPCR. Error bars show mean \pm SEM. P values obtained by Student's t-test. * $P<0.05$. d, The KDM3B-centered networks in $K d m 3 b^{+/+}$and $K d m 3 b^{+/-}$mouse retinas. The circles indicate individual nodes (i.e., regulator and target genes), and the line indicates the interaction between regulatory hubs and genes. The KDM3B-centered network is shown as a yellow circle in the center of networks. The differential expression nodes are represented by the blue circles (downregulated genes in $K d m 3 b^{+/}$) and red circles (upregulated genes in $K d m 3 b^{+/}$). e, The matrix of dissimilarity D's measured from each pair of networks. f, The plot of fraction of nodes that belong to the largest component in the network for link removal fraction $f$. All quantities are averaged over 300 realizations for each network. The error bars are estimated from all realizations. g, Schematic representation of KDM3B-centered network during mouse retinal aging. The integrated KDM3B-centered network with gene expression (mRNA), chromatin status (H3K9mel, $\mathrm{H} 3 \mathrm{~K} 9 \mathrm{me} 2$ ), protein was visualized in the cube. Subtle change of network strength in $K d m 3 b^{+/}$mouse retina leads to the breakdown of functional physiology in the cone photoreceptors. 


\section{Extended Data Fig. 1}

a

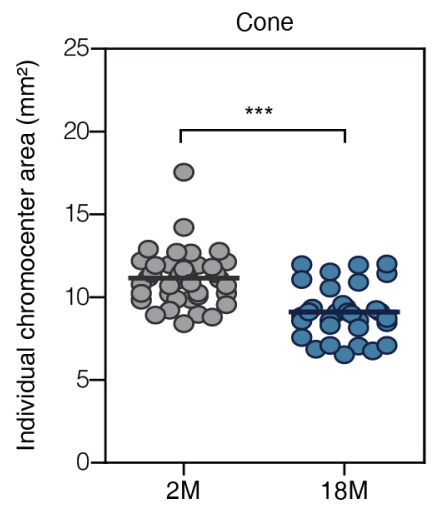

C

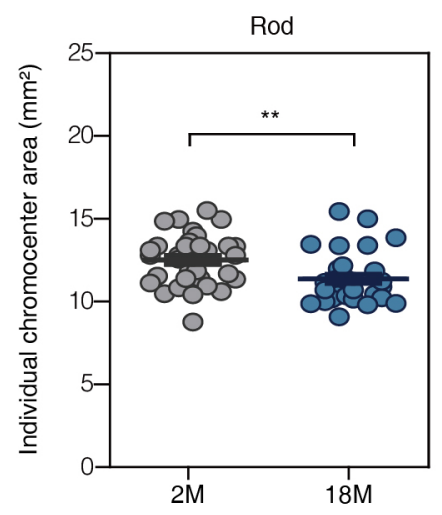

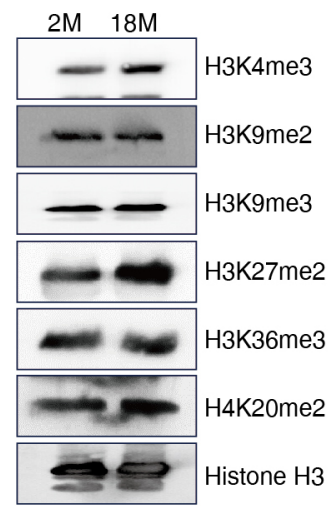

b
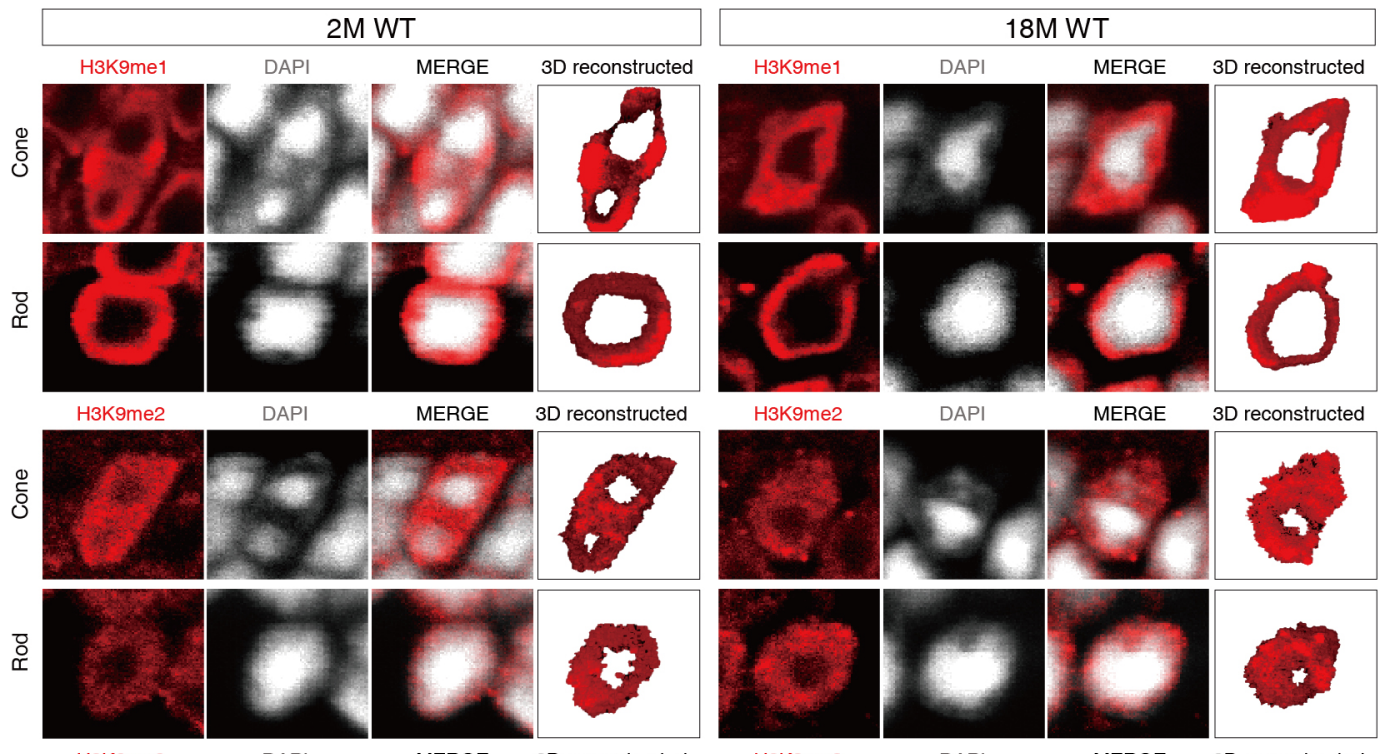

MERGE

3D reconstructed

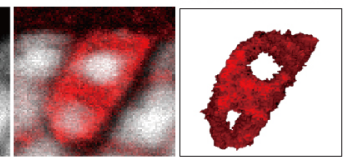

H3K9me2

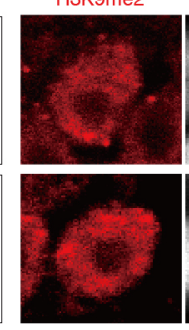

DAPI

MERGE

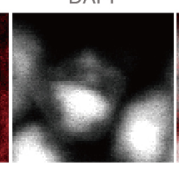

3D reconstructed
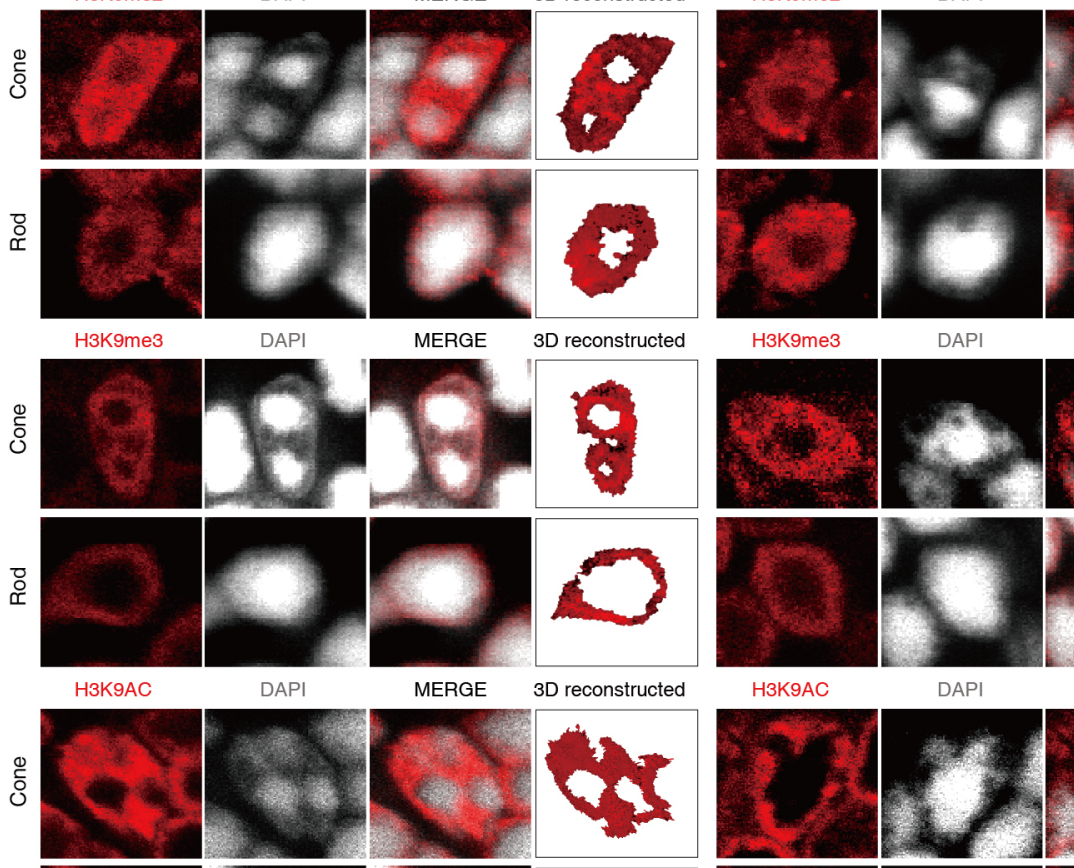

DAPI

MERGE

3D reconstructed
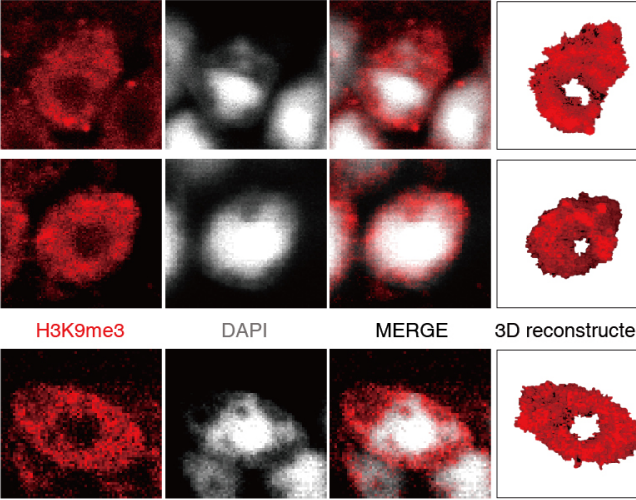

DAPI

MERGE
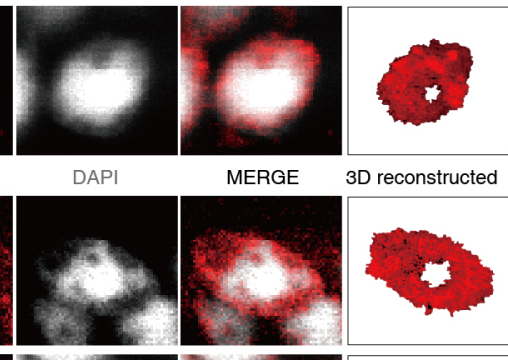

3D reconstructed
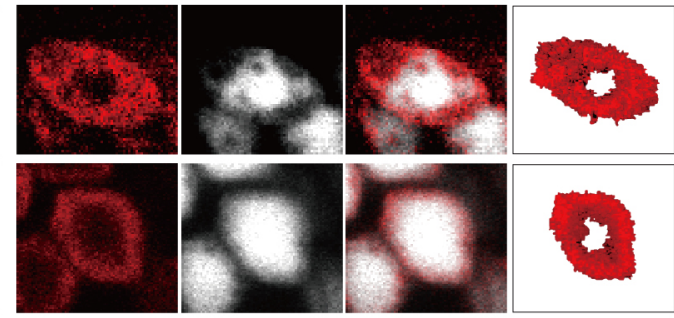

H3K9AC

DAPI

MERGE
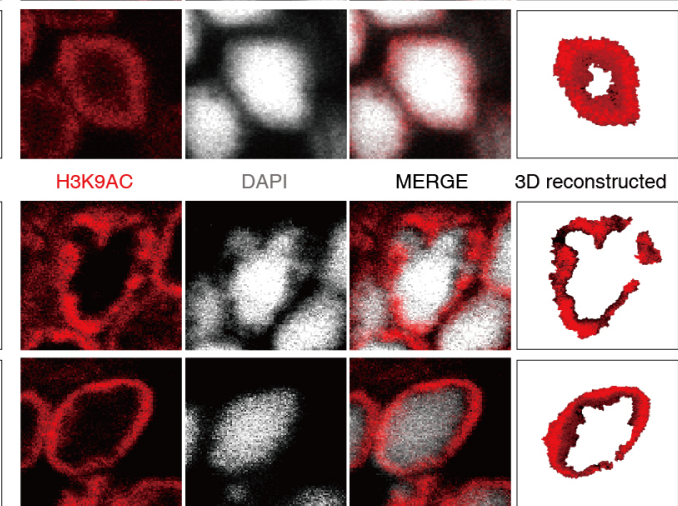

3D reconstructed
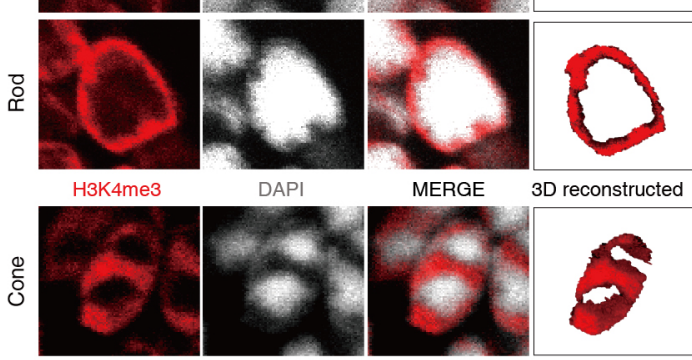

MERGE
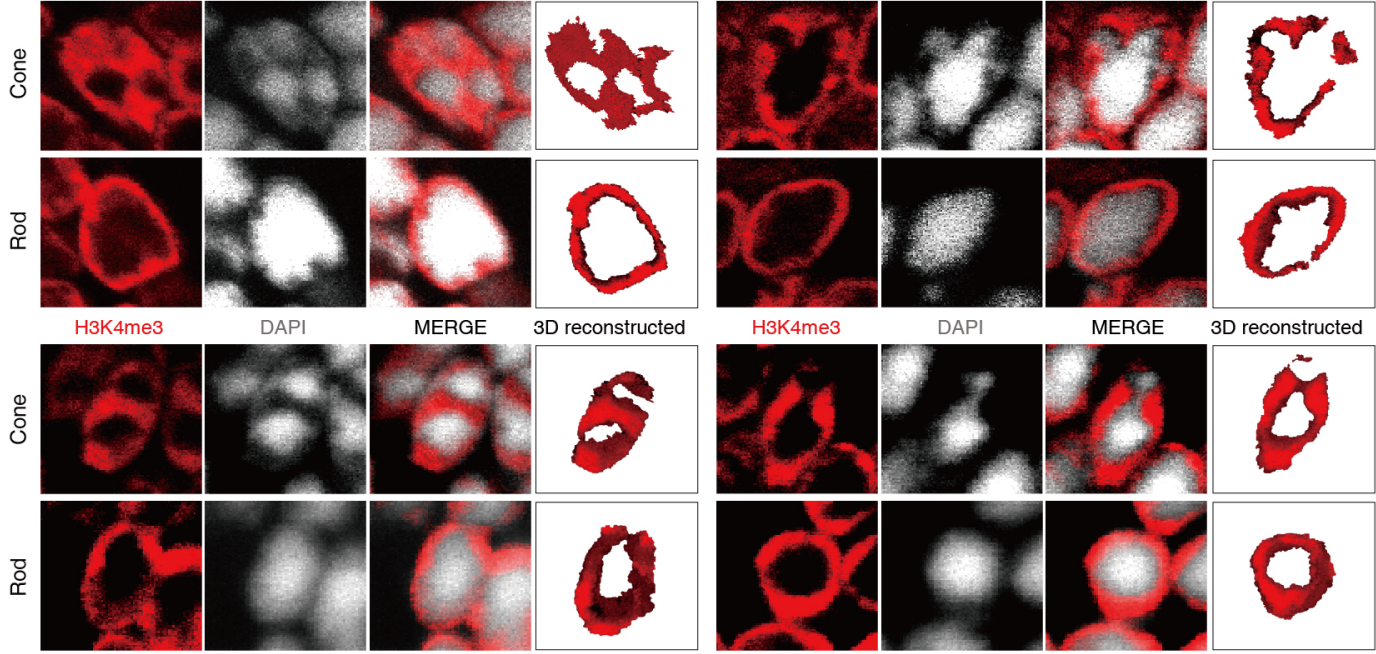

Extended Data Fig. 1 Characterization of heterochromatin organization during retinal aging. a, The area of chromocenter $\left(\mu \mathrm{m}^{2}\right)$ in cone and rod. b. Whole retinal lysates of 2- and 18-month-old $K d m 3 b^{+/+}$mice were immunoblotted using histone specific modification antibodies. Histone $\mathrm{H} 3$ antibody was used for internal loading control. c, Immunostaining of histone mark (red) and DAPI in 2- and 18-month-old $K d m 3 b^{+/+}$mouse retinas. 


\section{Extended Data Fig. 2}

a

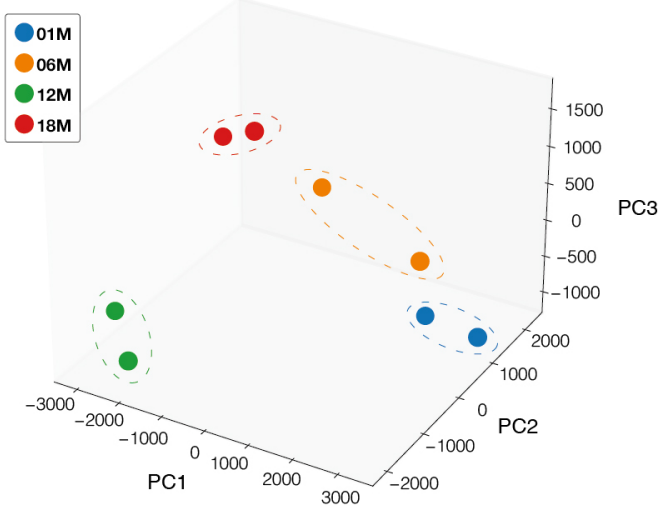

C

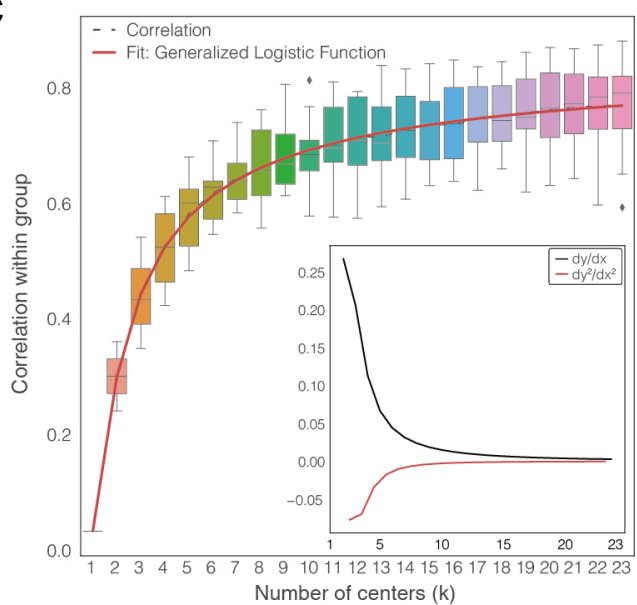

d

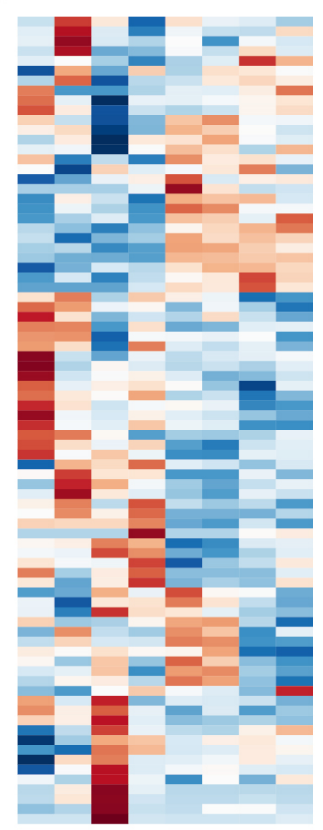

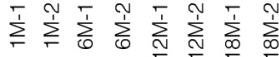

e

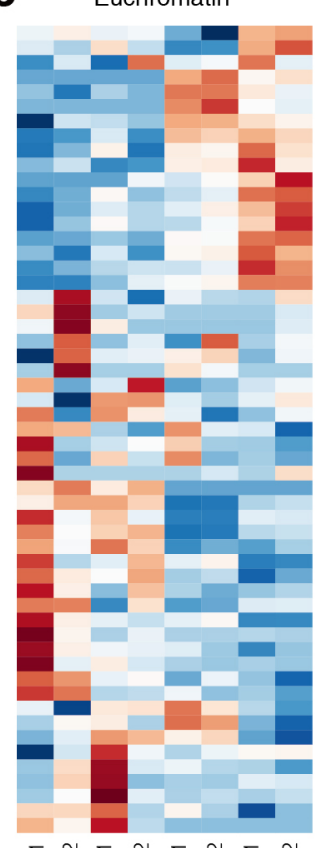

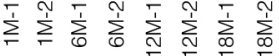

b

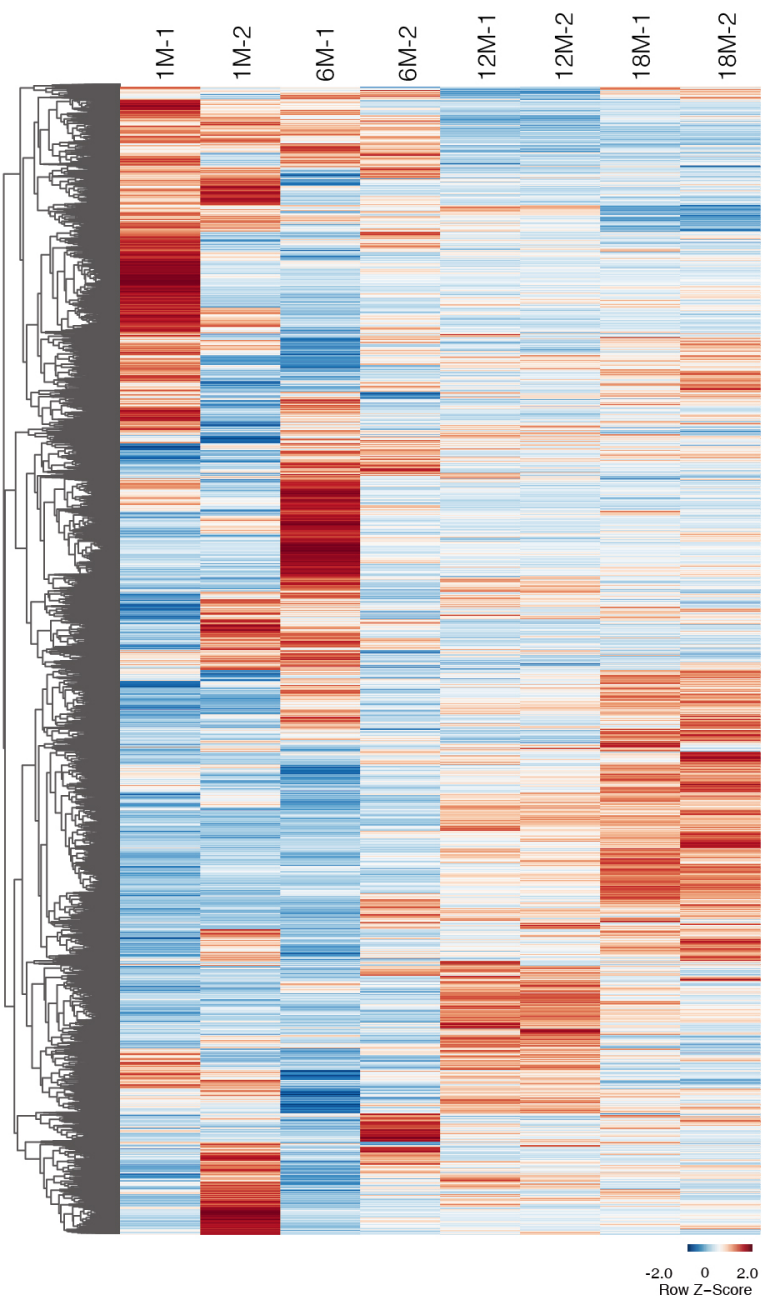

f

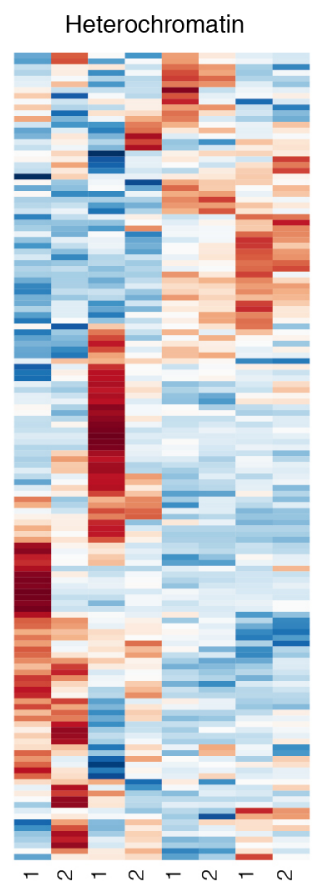

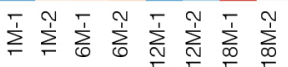

g

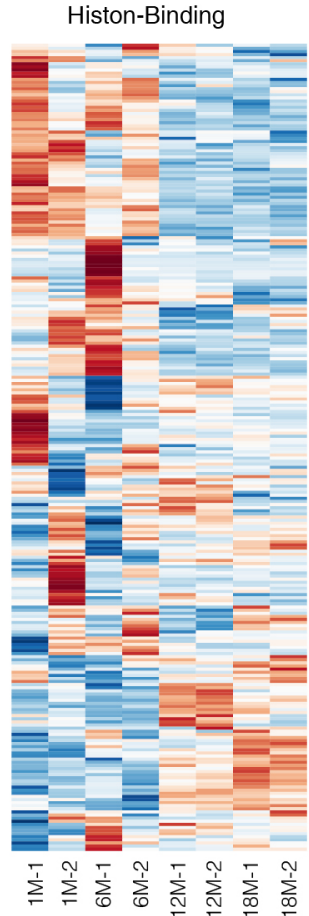

h

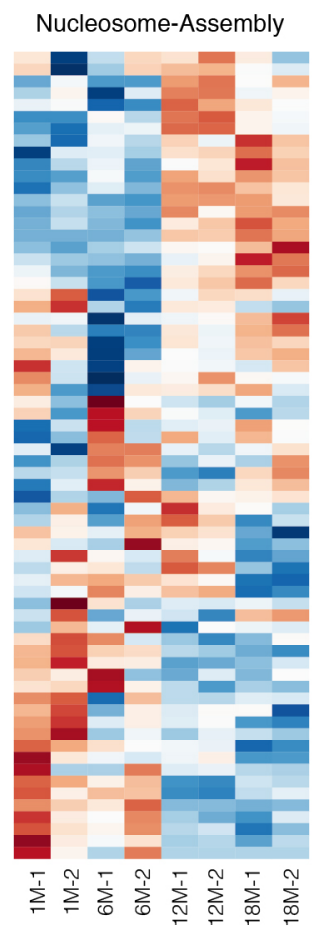

Extended Data Fig. 2 Transcriptomic profiling during retinal aging. a, PCA plot of RNA-seq from 1-, 6-, 12- and 18-months mouse retinas. $\mathbf{b}$, Hierarchically clustered heatmap of transcripts (a total of 19,484 annotated transcripts were prefiltered by FPKM $>1$ that were differentially expressed in 1-, 6-, 12- and 18-months mouse retinas). $\mathbf{c}$, Plot of correlation within group for the number of $\mathrm{k}$ centers and fitting the data with generalized logistic function. Inset of (c): Plots of first and second-order to the fitted function. d-h, Hierarchically clustered heatmap of transcripts involved in DNA-modification, euchromatin, heterochromatin, histone-binding, and nucleosome-assembly. 


\section{Extended Data Fig. 3}

a
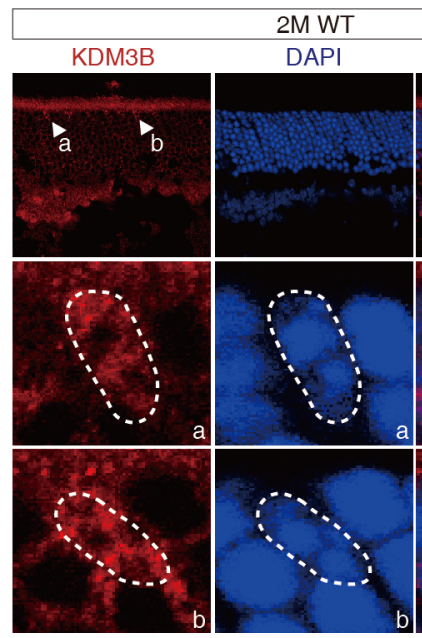

MERGE

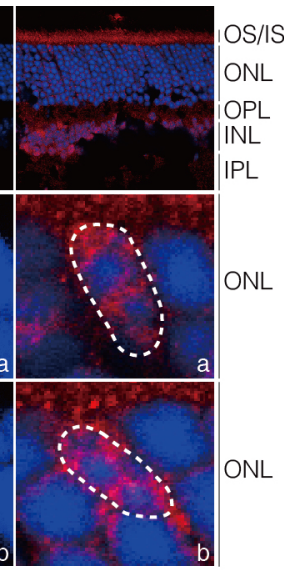

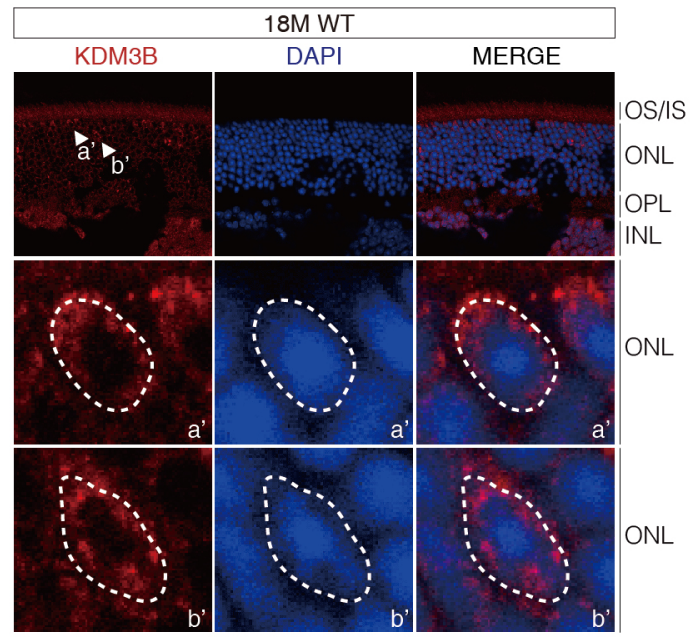

b

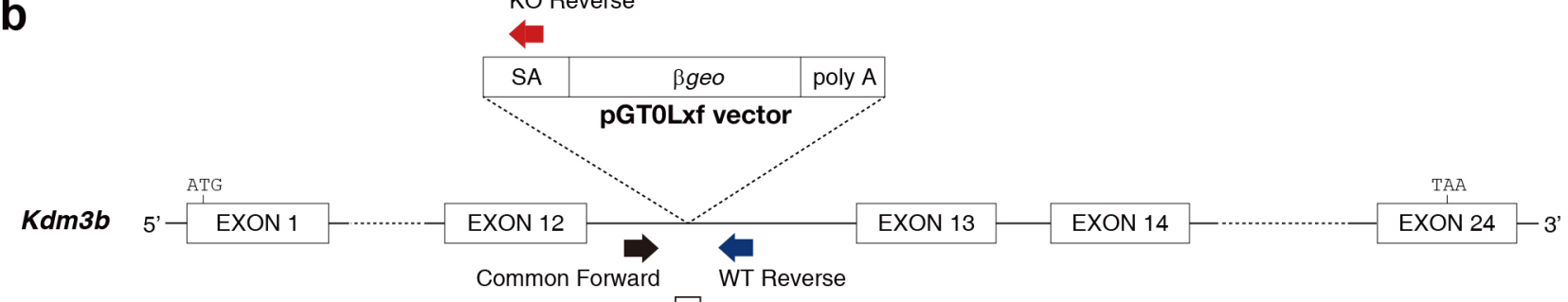

$\square$ Transcription

C

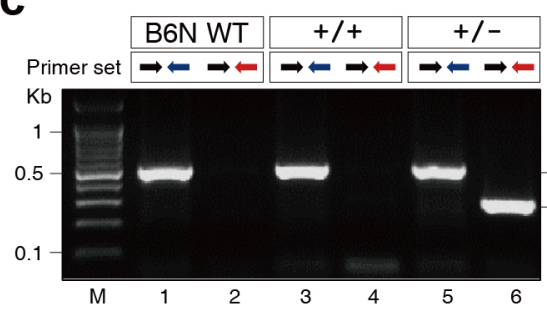

f

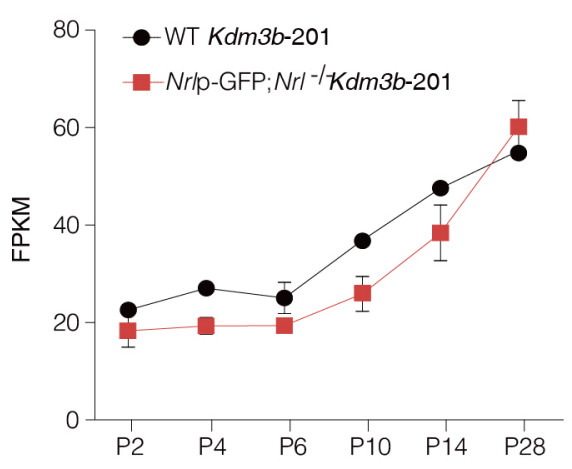

d

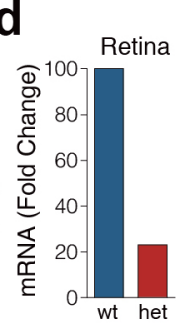

g

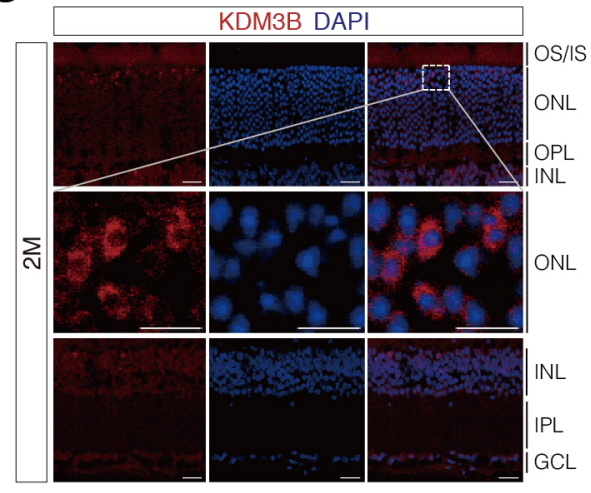

e
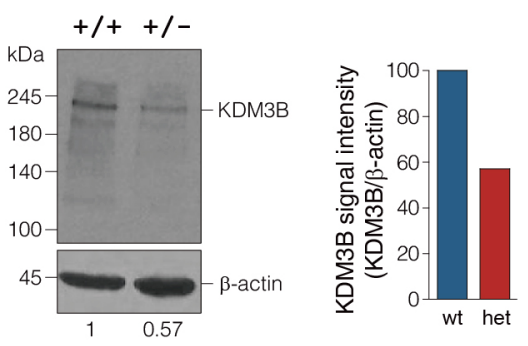

h
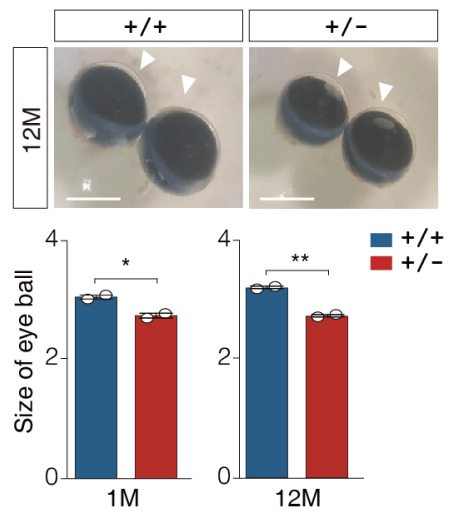

Extended Data Fig. 3 Generation of $\mathbf{K d m} 3 \boldsymbol{b}^{+/}$mice. a, Immunostaining of KDM3B (red) and DAPI in 2- and 18-month-old $K d m 3 b^{+/+}$mouse retinas. b. Gene trap vector (pGT0Lxf) was inserted into the intron region between exon 12 and exon 13. c, Genotyping of $K d m 3 b^{+/+}$and $K d m 3 b^{+/-}$ mice was performed by conventional PCR analysis using isolated genomic DNA (gDNA) from mouse tails representing each genotype. The bands of $475 \mathrm{bp}$ and $282 \mathrm{bp}$ correspond to WT and mutant alleles, respectively. d, qRT-PCR analysis of $K d m 3 b$ mRNA expression (fold-change) in retina, brain, and liver of $K d m 3 b^{+/+}$and $K d m 3 b^{+/}$mice. e, Whole retinal lysates of $K d m 3 b^{+/+}$and $K d m 3 b^{+/}$mice were immunoblotted using anti-KDM3B antibody (left). $\beta$-actin was used for internal loading control. Signal intensity of KDM3B was quantified using ImageJ software (right). f, Expression level of $K d m 3 b$ transcripts in whole retina (WT) and s-cone-like cells ( $\left.N r l p-G F P ; N_{r} t^{-/}\right)$plotted for six developmental time points (P2, P4, P6, P10, P14, and P28) using public RNA-sequencing data (GSE 74660). g, Immunostaining of KDM3B (red) and DAPI in 2-month-old $K d m 3 b^{+/+}$mouse retinas. Scale bar: $20 \mu \mathrm{m}$. High magnification image in the white box showing KDM3B (red) staining in cones (middle panel). Scale bar: $10 \mu \mathrm{m}$. h, Eyeballs were enucleated from 12-month-old $K d m 3 b^{+/+}$and $K d m 3 b^{+/-}$mice. White arrows indicate the abnormal cornea phenotype. Scale bar: $2 \mathrm{~mm}$. The sizes of eyeballs in 1- and 12-month-old $K d m 3 b^{+++}$and $K d m 3 b^{+/}$mice was measured. Error bars show mean \pm SEM. $P$ values obtained by Student's t-test. ** $P<0.01, * P<0.05$. 
a
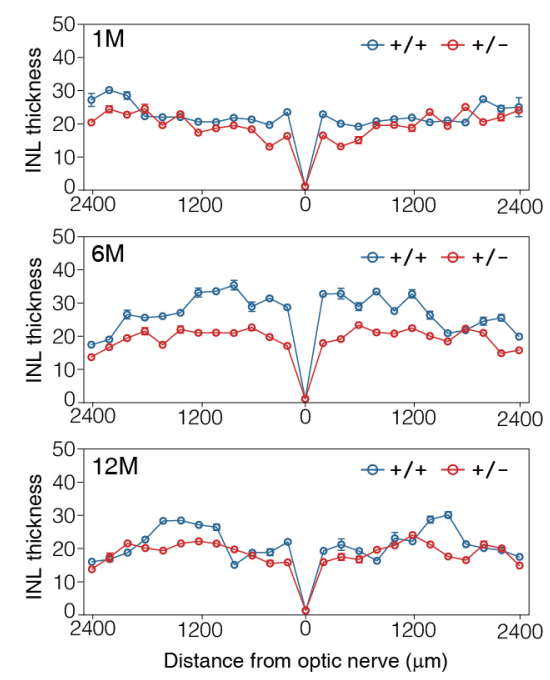

C

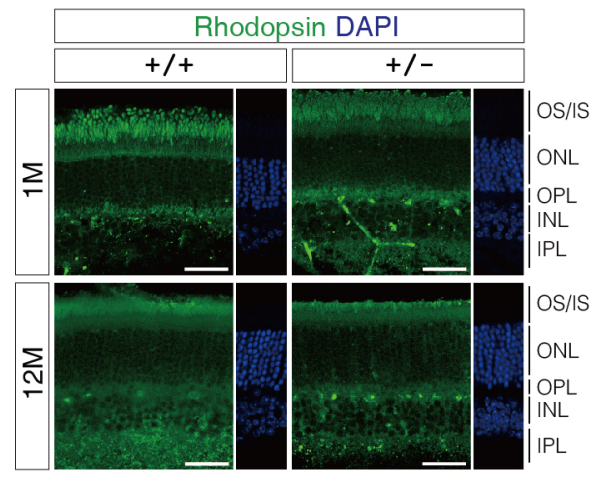

b
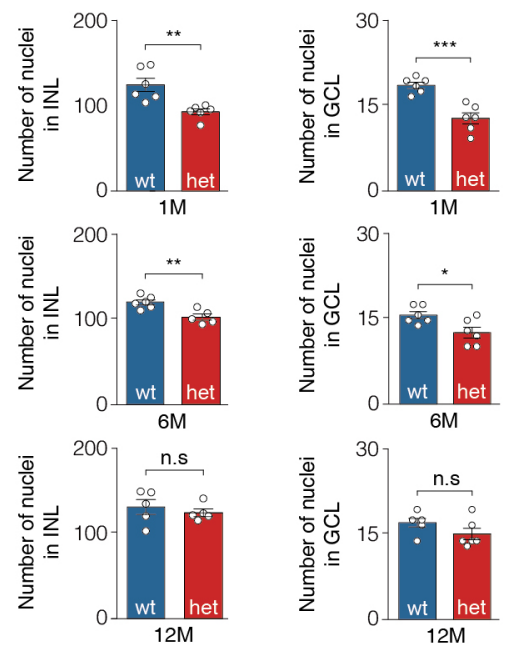

d

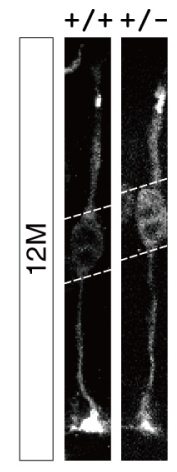

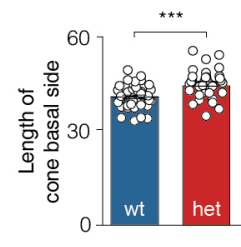

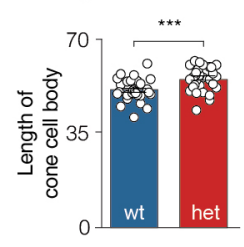

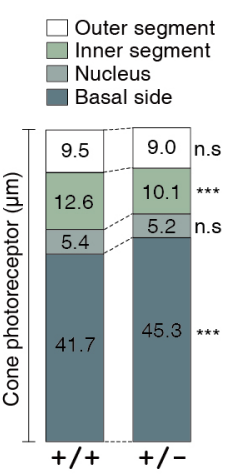

Extended Data Fig. 4 The morphological changes of rod and cone photoreceptors in $K d m 3 b^{+/}$mouse retinas. a, Thickness of INL was measured along the vertical meridians of the retinas of 1-, 6-, and 12-month-old $K d m 3 b^{+/+}$and $K d m 3 b^{+/}$mice $(\mathrm{n} \geq 6)$. b. The number of nuclei in INL was quantified in $100 \mu^{2}$ of retina (left) and linearized GCL in $100 \mu \mathrm{m}$ of retina (right). c, Immunostaining of rhodopsin (Rho) in 1 and 12-month-old $K d m 3 b^{+++}$and $K d m 3 b^{+/}$mouse retinas. Scale bar: $40 \mu \mathrm{m}$. d, Measurements of the length of cone basal side $(\mu \mathrm{m})$ and cell body $(\mu \mathrm{m})$ by ImageJ software. Error bars show mean \pm SEM. $P$ values obtained by Student's t-test. $* * * P<0.001, \mathrm{n}$.s, not significant. 


\section{Extended Data Fig. 5}

a

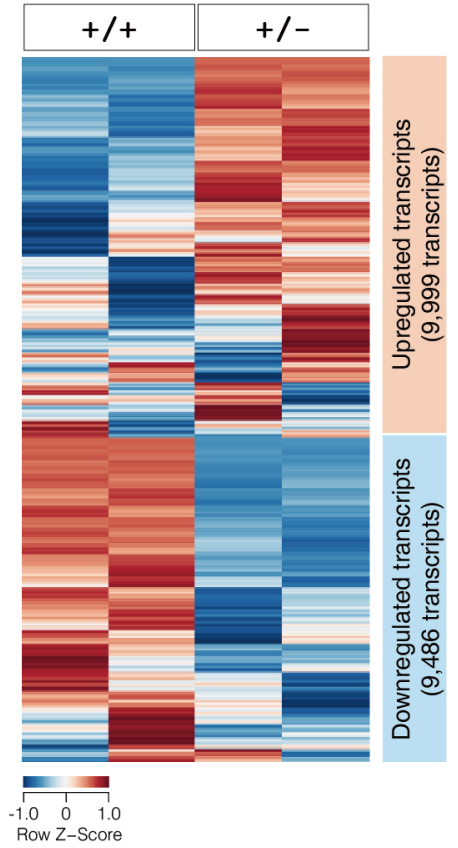

b

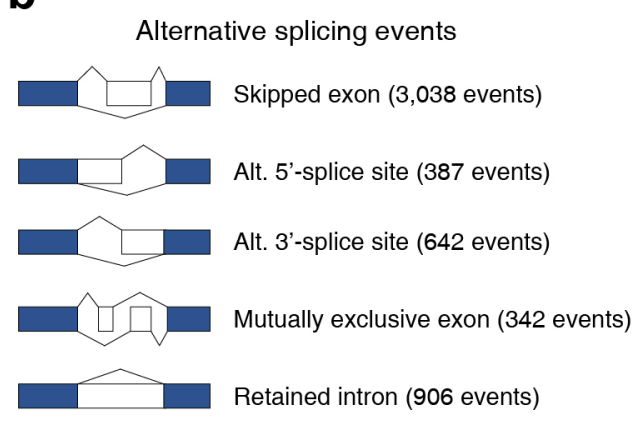

d

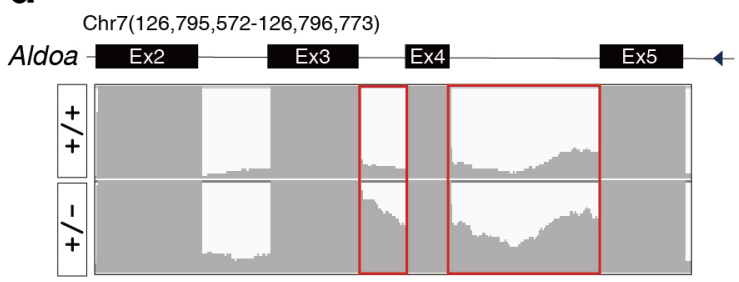

C

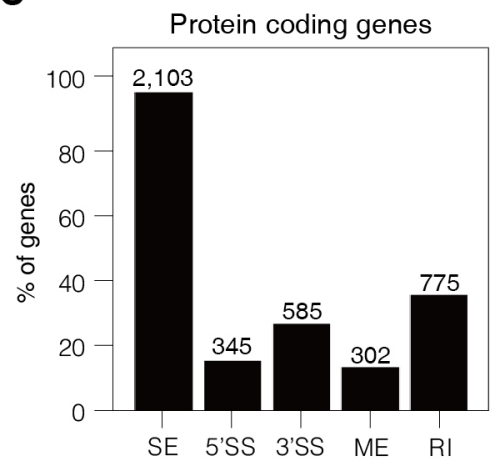

Extended Data Fig. 5 Alternative splicing in $\mathbf{K d m} \mathbf{3} \boldsymbol{b}^{+/}$mouse retinas. a, Hierarchically clustered heatmap of transcripts (a total of 19,485 annotated transcripts (pre-filtered by FPKM > 1) that were differentially expressed in $K d m 3 b^{+/+}$and $K d m 3 b^{+/}$mouse retinas). b, Alternative splicing events were analyzed with Rmats (v4.1), which identified 5,315 genes having alternative splicing events (P value $<$ 0.05). Types of alternative splicing events detected and frequencies in group-wise comparisons. c, Protein coding genes obtained from alternative splicing events indicated as the percentages of counted genes. d, Differential retained introns between $K d m 3 b^{+/+}$and $K d m 3 b^{+/-}$ mouse retinas. The genomic area of the Aldoa gene is shown, which comprises eight exons and seven introns, and RNA-sequencing coverage plots of $K d m 3 b^{++}$and $K d m 3 b^{+/}$in the area of exons two through five. Red box indicates the clear retained intron region in $K d m 3 b^{+-}$ mouse retinas. 


\section{Extended Data Fig. 6}

a

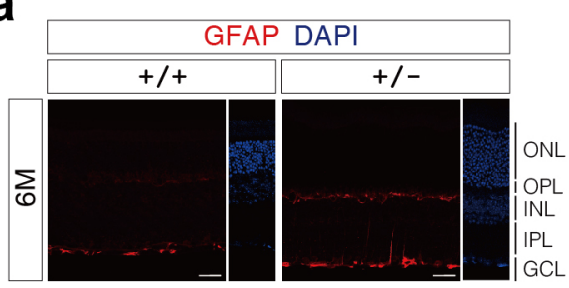

b

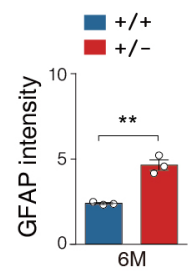

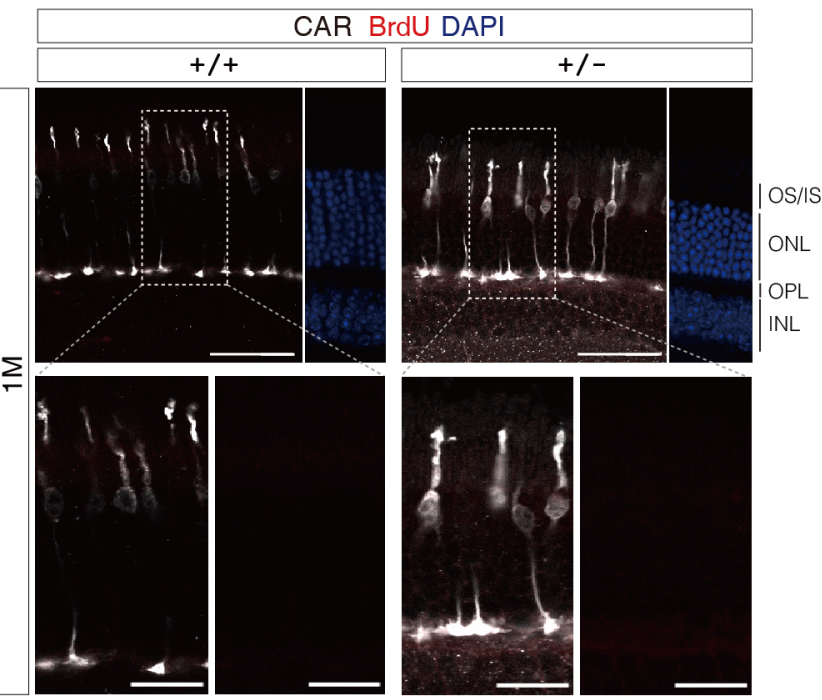

d
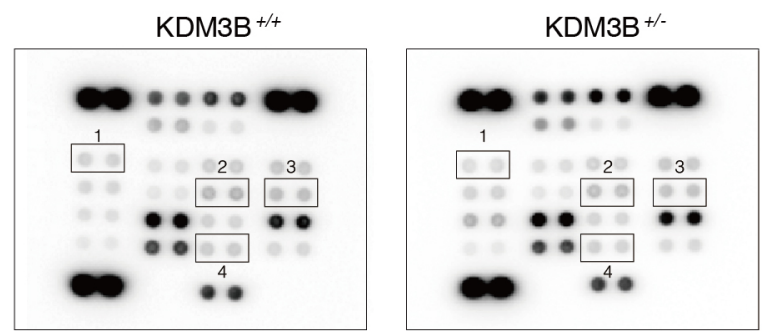

Extended Data Fig. 6 Increase of apoptotic cell death in cones in $K \boldsymbol{d} \mathbf{m} 3 \boldsymbol{b}^{+/}$retinas. a, Immunostaining of GFAP in 6-month-old $K d m 3 b^{+/+}$and $K d m 3 b^{+/}$mouse retinas (left). Scale bar: $20 \mu \mathrm{m}$. GFAP-intensity of Müller glial cells was measured by ImageJ in 6-month-old $K d m 3 b^{+/+}$and $K d m 3 b^{+/}$mouse retinas (right). b. Apoptotic cells were detected by TUNEL assay (BrdU positive cells) in 12-month-old $K d m 3 b^{+/+}$and $K d m 3 b^{+/}$mouse retinas. Scale bar: $50 \mu \mathrm{m}$. BrdU-positive cones in red dashed box. c, d, The entire apoptosis proteome profile array in 8-month-old $K d m 3 b^{+/+}$and $K d m 3 b^{+/}$mouse retinas. 


\section{Extended Data Fig. 7}

a

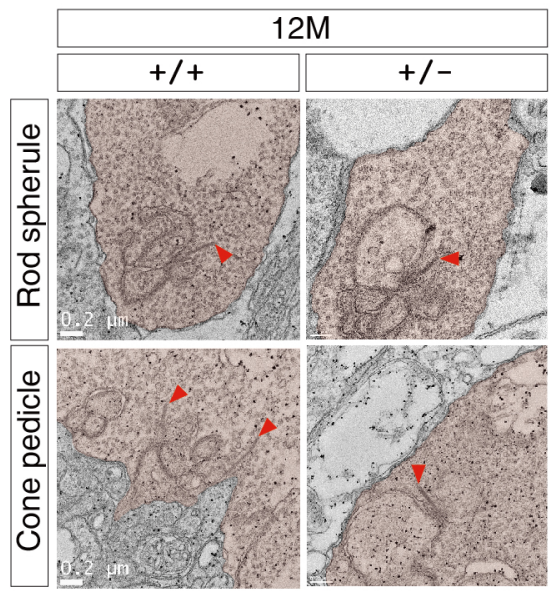

b
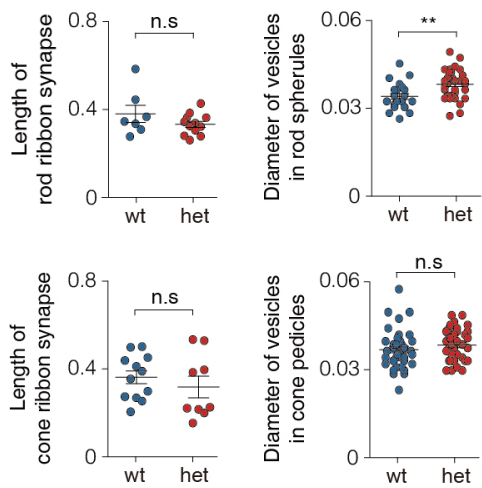

C

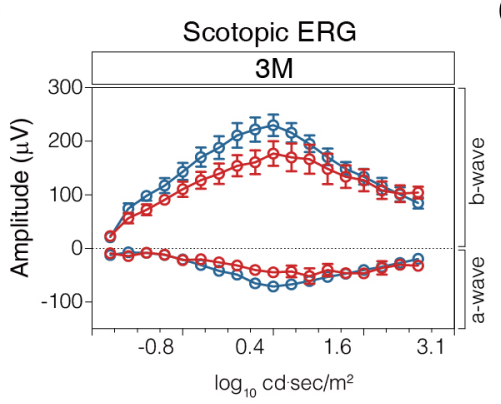

f

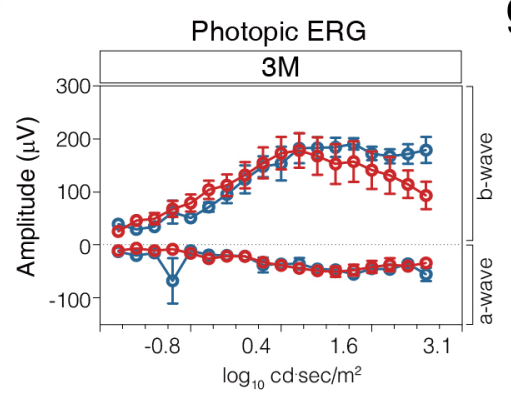

d

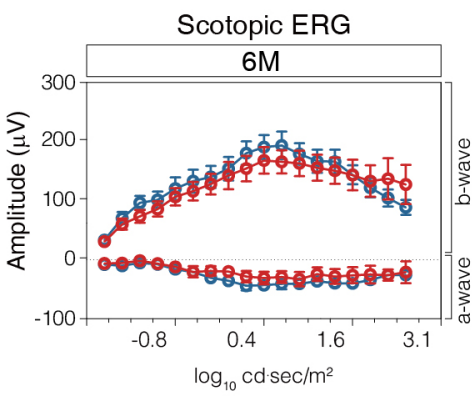

g

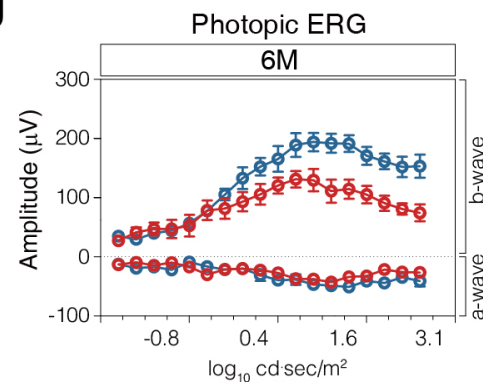

e

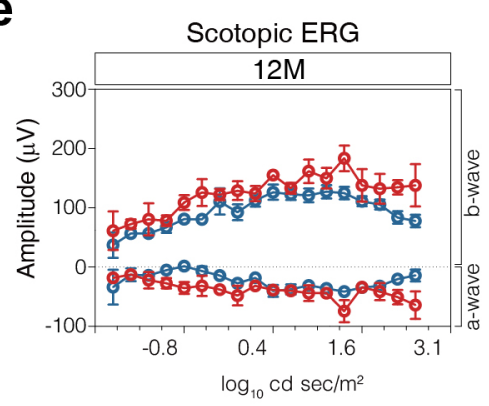

h

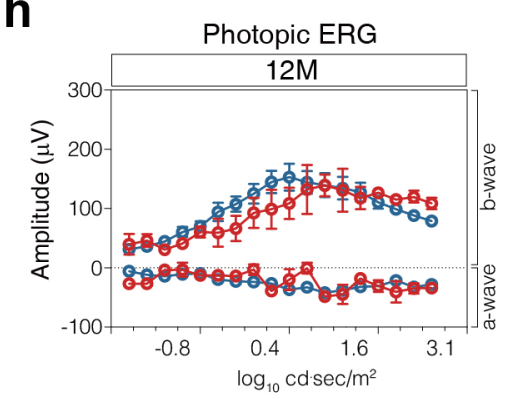

i

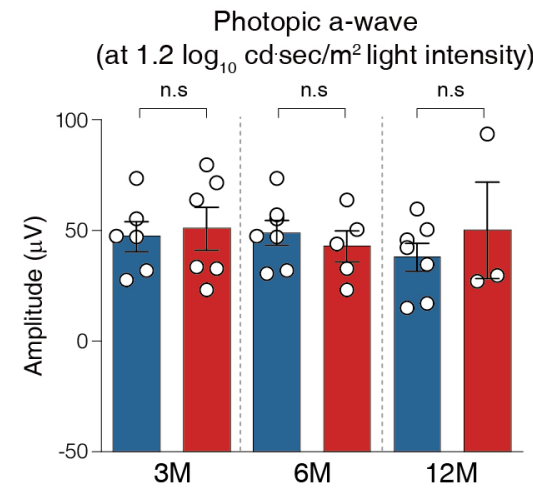

Photopic b-wave

(at $1.2 \log _{10} \mathrm{~cd} \cdot \mathrm{sec} / \mathrm{m}^{2}$ light intensity)

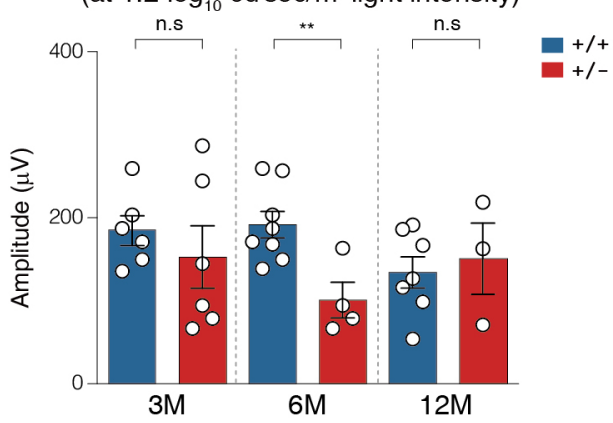

Extended Data Fig. 7 The alteration of synaptic morphology of cone photoreceptors in $\boldsymbol{K d m} \mathbf{3} \boldsymbol{b}^{+/}$mice retina. a, TEM images of ribbon synapse in rod spherules and cone pedicles of 12-month-old $K d m 3 b^{+/+}$and $K d m 3 b^{+/-}$mice. Scale bar: $0.2 \mu \mathrm{m}$. b, Measurements of the length of ribbon synapses $(\mu \mathrm{m})$ (left) and diameter of vesicles $(\mu \mathrm{m})$ (right) by ImageJ software. Error bars show mean \pm SEM. P values obtained by Student's t-test. ** $\mathrm{P}<0.01$, n.s, not significant. c-e, Representative scotopic ERGs elicited from 3- (c), 6- (d), and 12- (e) month-old $K d m 3 b^{+/+}$and $K d m 3 b^{+/}$mice. f-h, Representative photopic ERGs elicited from 3- (f), 6- (g), and 12- (h) month-old $K d m 3 b^{+/+}$ and $K d m 3 b^{+/-}$mice. i, Quantification of ERG responses at $1.2 \log 10 \mathrm{~cd} . \mathrm{sec} / \mathrm{m} 2$ light intensity from 3-, 6-, and 12-month-old $K d m 3 b^{+/ /}$and $K d m 3 b^{+/-}$mice. Graphs show the mean \pm SEM, and data are representative of $\mathrm{n}>3$ per each genotype. $P$ values obtained by Student's t-test. ** $P<0.01$, n.s, not significant. 


\section{Extended Data Fig. 8}

a
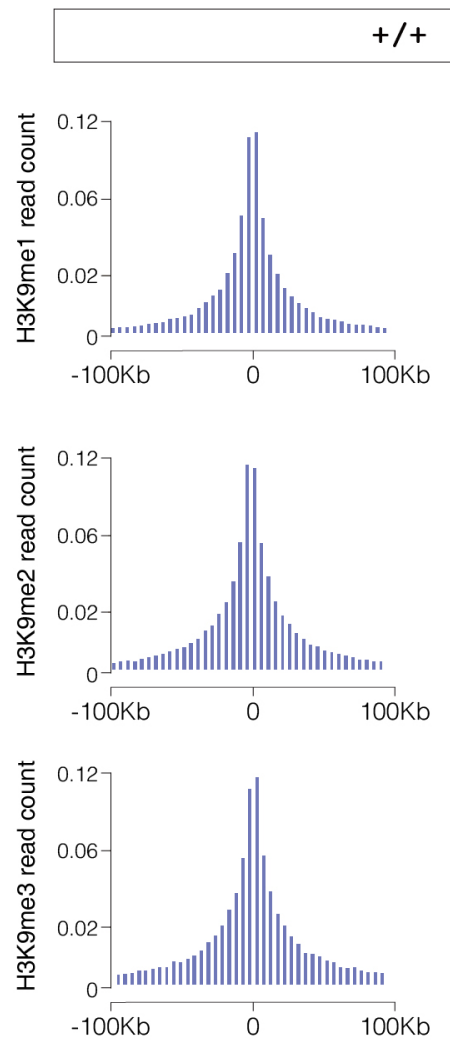

b
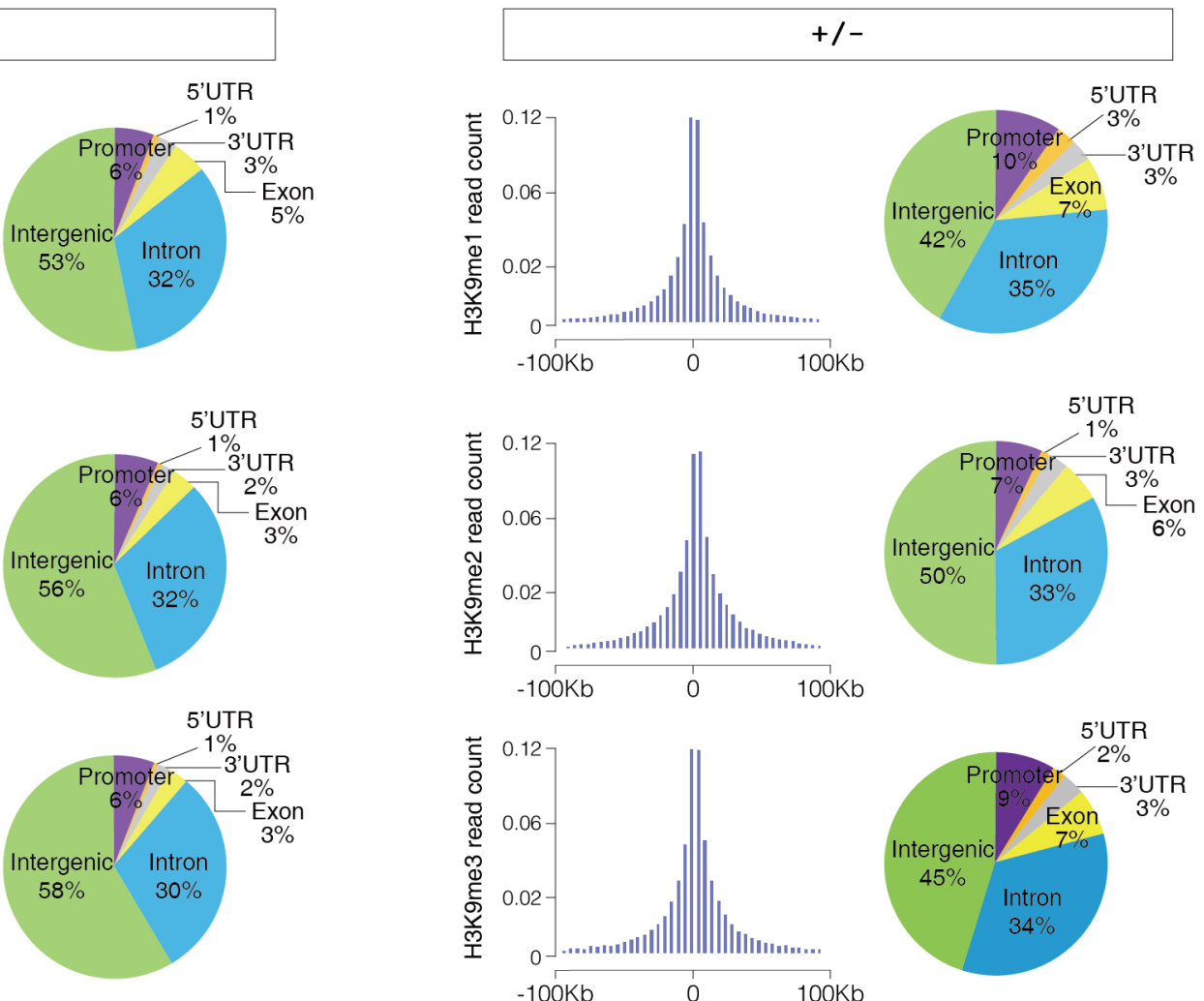
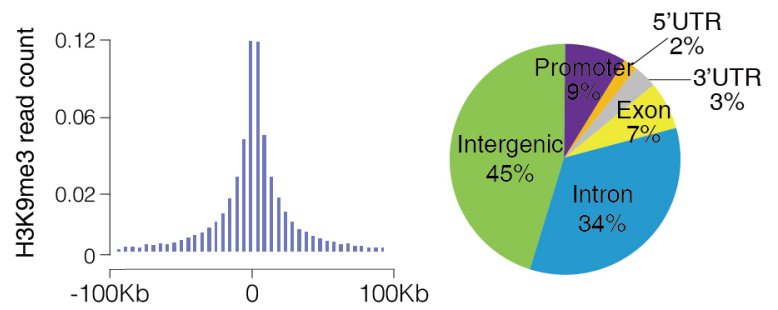

Extended Data Fig. 8 KDM3B is required for global H3K9 demethylation in mouse retinas. a, b, Bar graph shows the distribution of H3K9me1, H3K9me2, and H3K9me3 peaks around transcriptional start sites (left). Pie chart of H3K9me1, H3K9me2, and H3K9me3 enrichment distribution at genomic loci (right) in $K d m 3 b^{+/+}$(a) and $K d m 3 b^{+/}$(b) mouse retinas. 


\section{Extended Data Fig. 9}

a
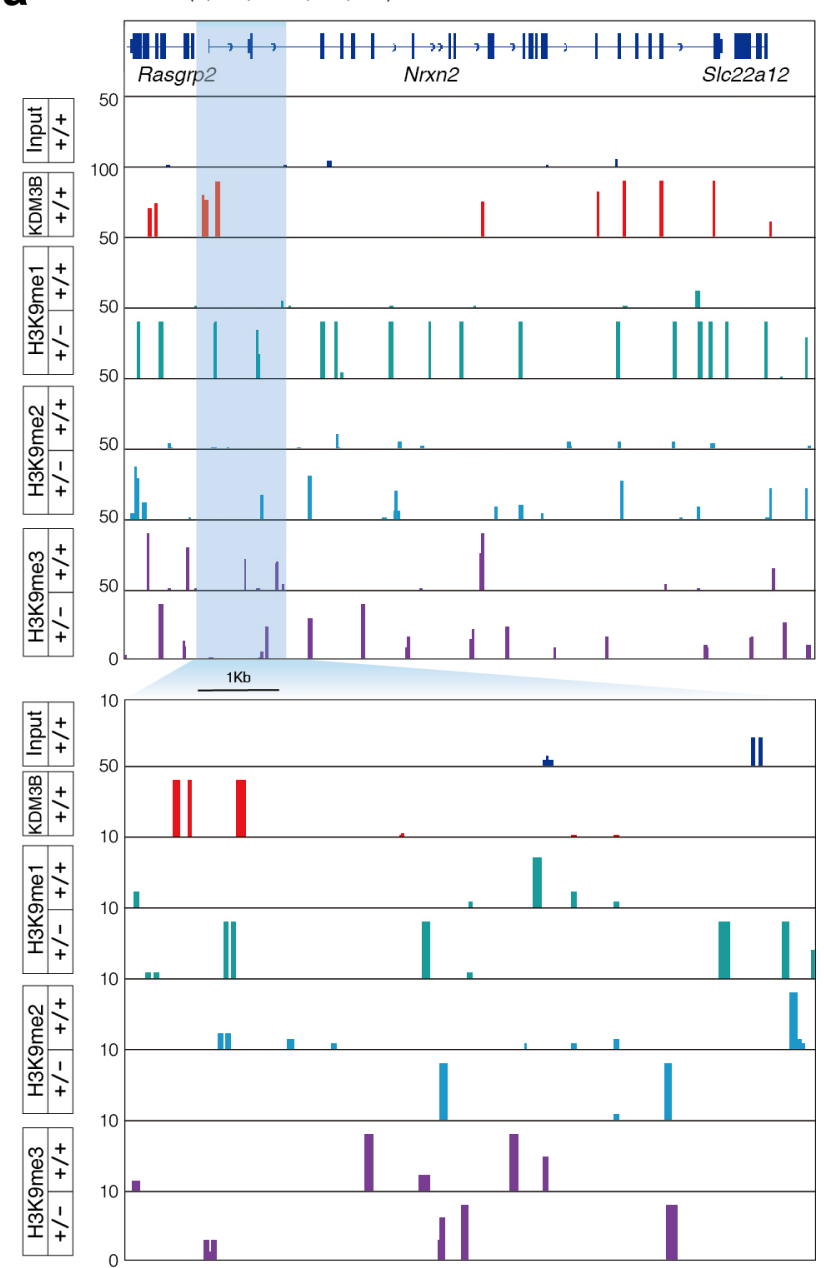

C

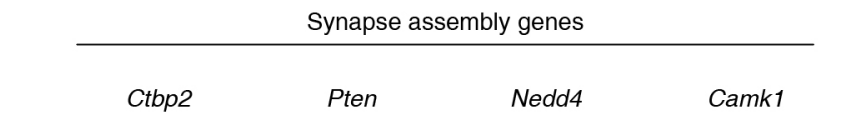

b $\quad \operatorname{Chr15}(38,887,786-39,981,637)$
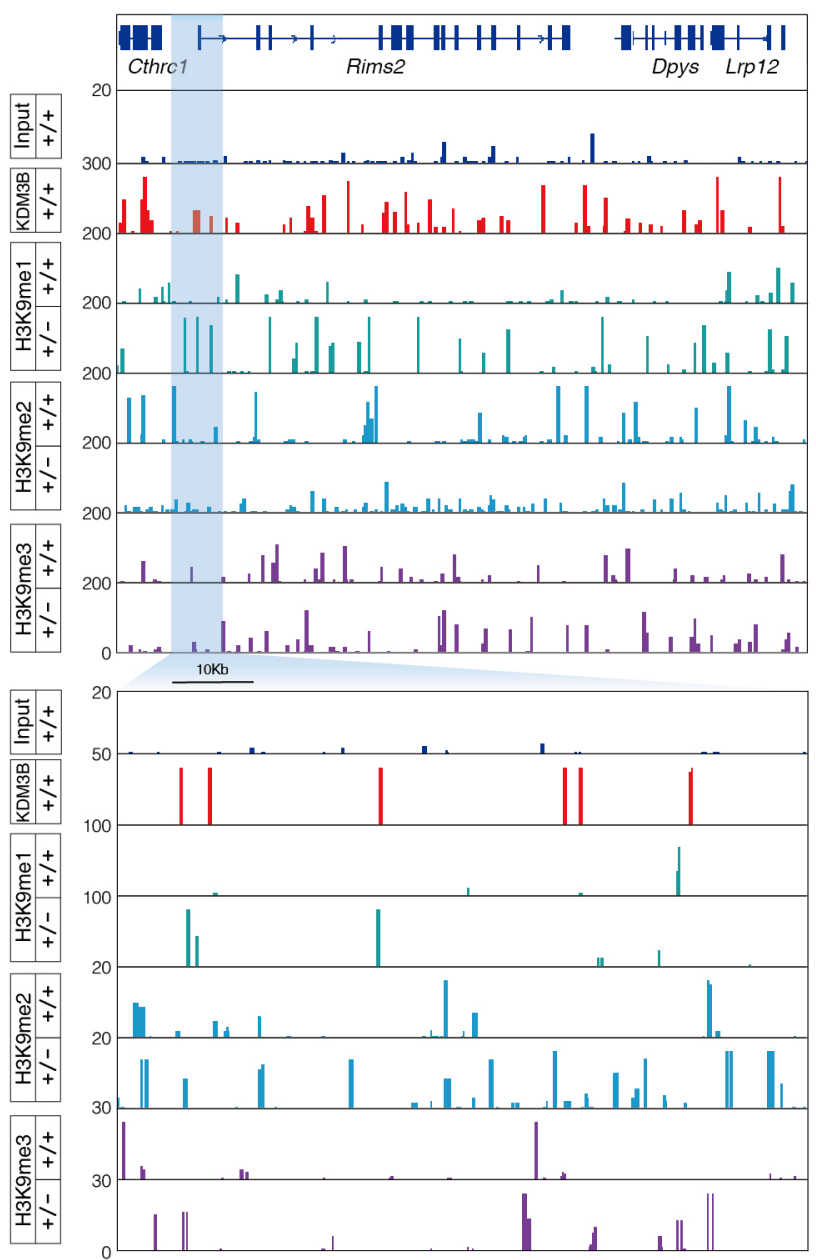

Vesicle transport genes

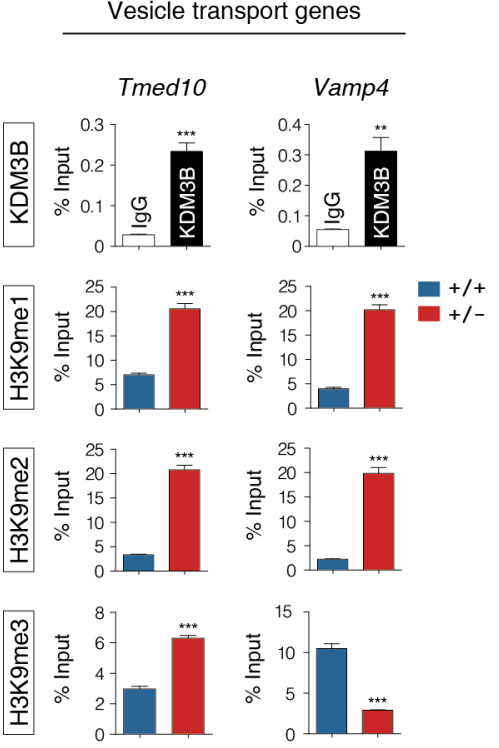

Extended Data Fig. 9 KDM3B regulates synapse assembly genes via erasing H3K9me1 and H3K9me2 marks. a, b, ChIP-seq tracks of $\mathrm{KDM} 3 \mathrm{~B}$ in $K d m 3 b^{+/ /}$and $\mathrm{H} 3 \mathrm{~K} 9 \mathrm{mel}$, H3K $9 \mathrm{me} 2$, and $\mathrm{H} 3 \mathrm{~K} 9 \mathrm{me} 3$ peaks in $K d m 3 b^{+/+}$and $K d m 3 b^{+/}$mouse retinas along the $N r \times n 2$ (a) and Rims2 (b) locus. Selected genomic elements (shaded with light cyan) indicate KDM3B ChIP-seq peaks at Nrxn2 and Rims2 promoter regions. c, Recruitment and presence of $\mathrm{KDM} 3 \mathrm{~B}, \mathrm{H} 3 \mathrm{~K} 9 \mathrm{mel}, \mathrm{H} 3 \mathrm{~K} 9 \mathrm{me} 2$, and $\mathrm{H} 3 \mathrm{~K} 9 \mathrm{me} 3$ at indicated target gene promoters were validated by ChIP-qPCR. Error bars show mean \pm SEM. $P$ values obtained by Student's t-test. $* * * P<0.001, * * P<0.01$, n.s, not significant. 


\section{Extended Data Fig. 10}

a

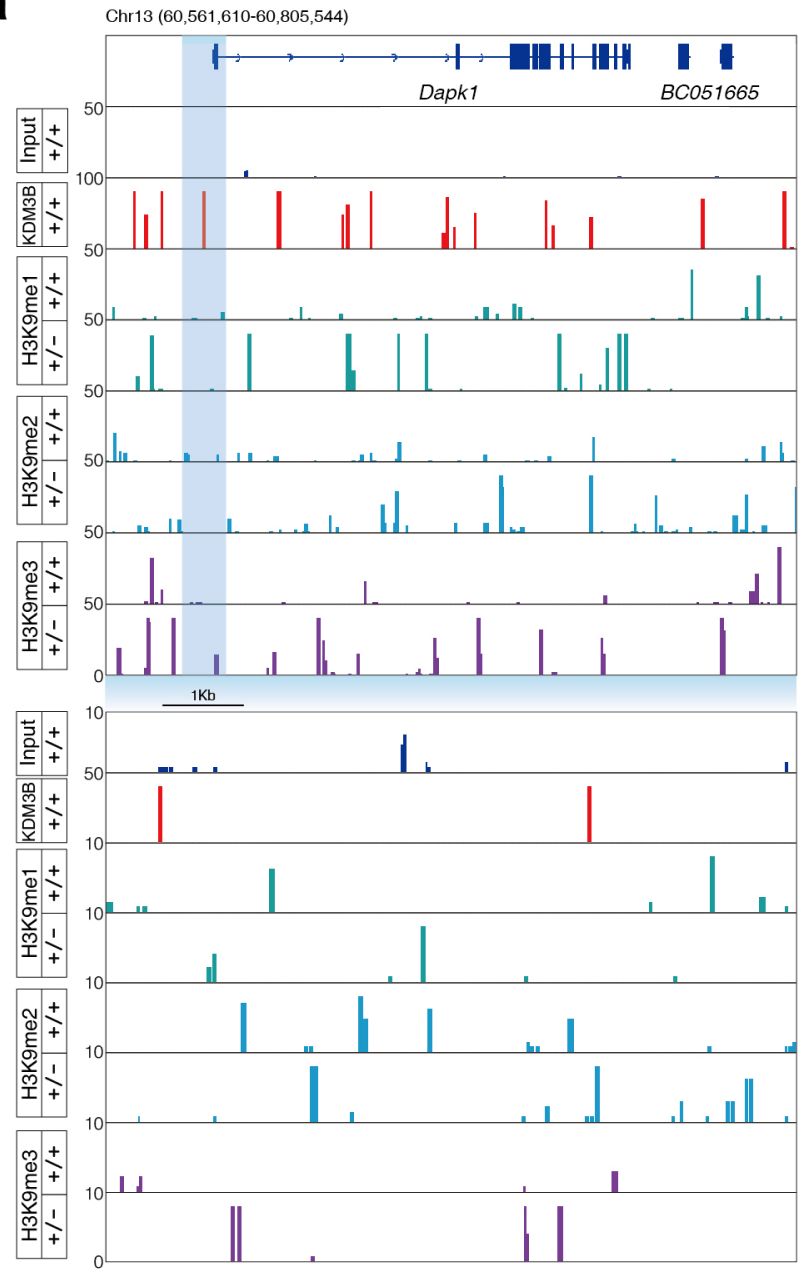

b

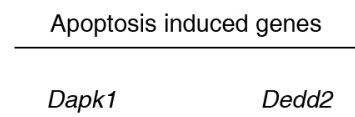

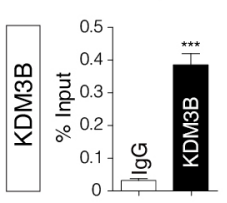

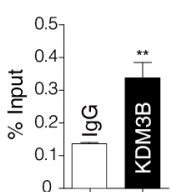

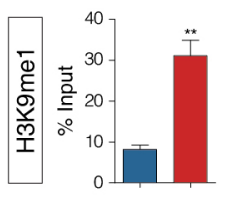

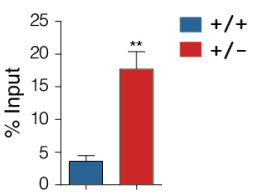

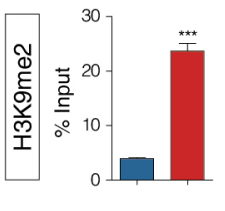

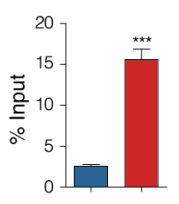

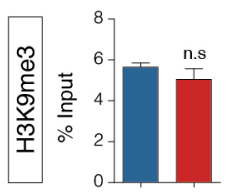

Extended Data Fig. 10 KDM3B regulates synapse assembly genes via erasing H3K9me1 and H3K9me2 marks. a, ChIP-seq tracks of KDM3B in $K d m 3 b^{+/+}$and H3K9mel, H3K9me2, and H3K9me3 peaks in $K d m 3 b^{+/+}$and $K d m 3 b^{+/}$mouse retinas along the Dapkl locus. Selected genomic element (shaded with light cyan) indicates KDM3B ChIP-seq peaks at Dapkl promoter region. b, Recruitment and presence of $\mathrm{KDM} 3 \mathrm{~B}, \mathrm{H} 3 \mathrm{~K} 9 \mathrm{mel}$, H3K9me2, and $\mathrm{H} 3 \mathrm{~K} 9 \mathrm{me} 3$ at apoptotic gene promoters were validated by ChIP-qPCR. Error bars show mean \pm SEM. $P$ values obtained by Student's t-test. $* * * P<0.001$, $* * P<0.01$, n.s, not significant. 


\section{Extended Data Fig. 11}

a

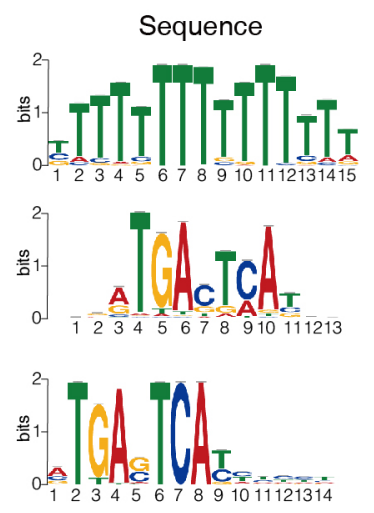

C

e

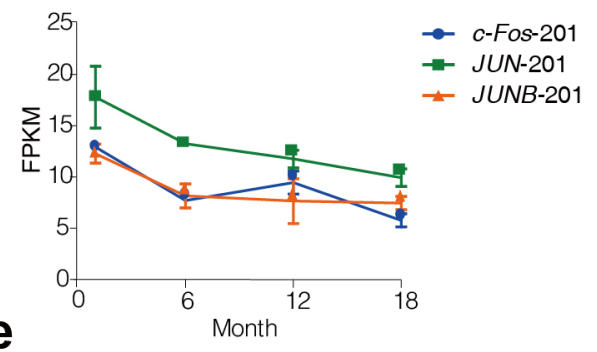

Motif $P$-value

ZNF384 3.45e-05

JUN $\quad 1.16 \mathrm{e}-04$

FOS::JUNB $\quad 2.85 \mathrm{e}-04$

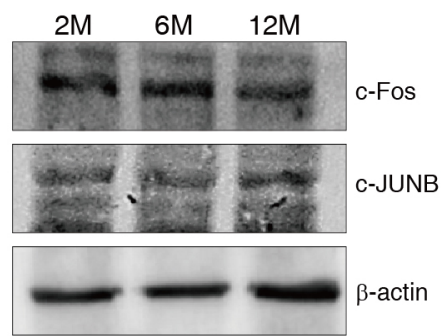

d
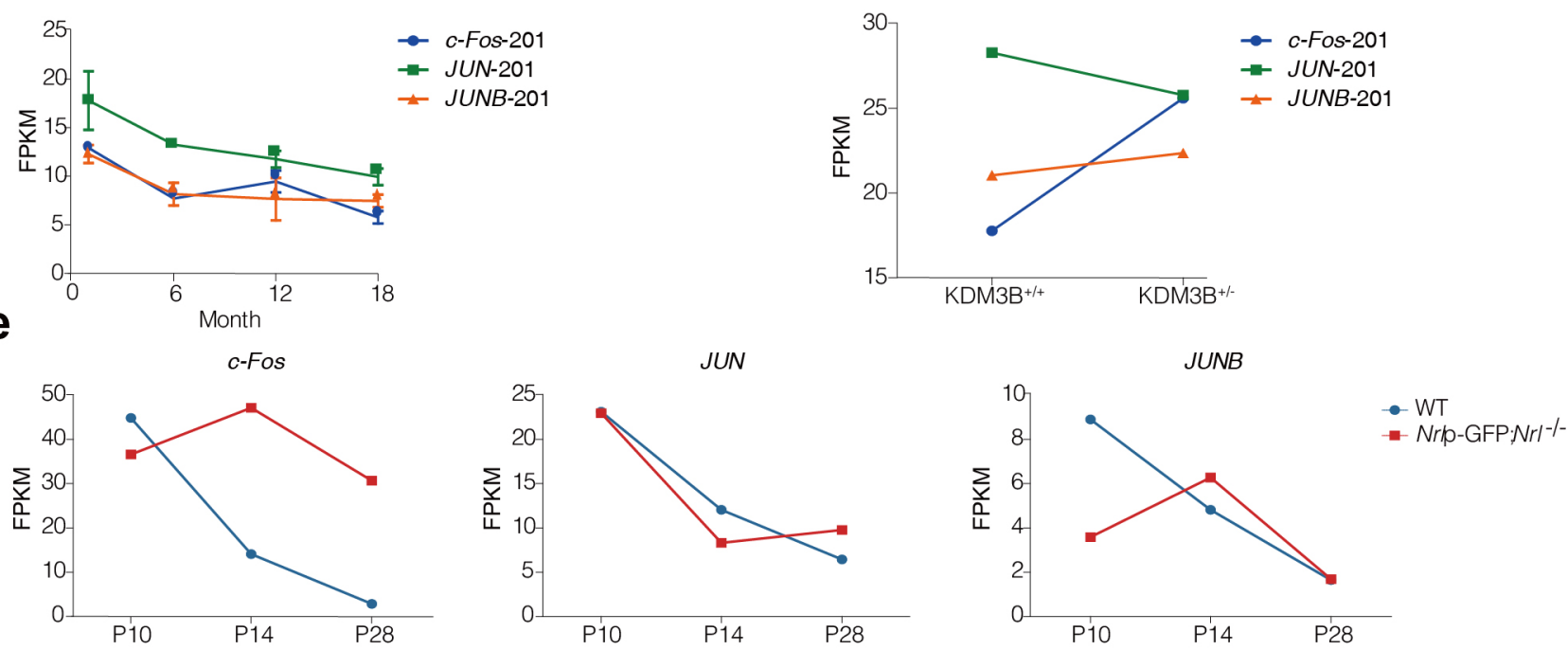

f

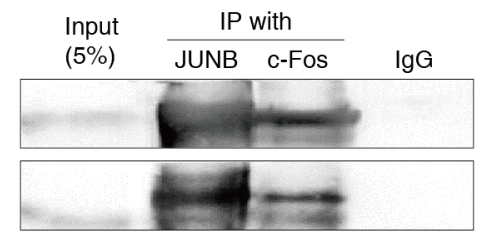

g
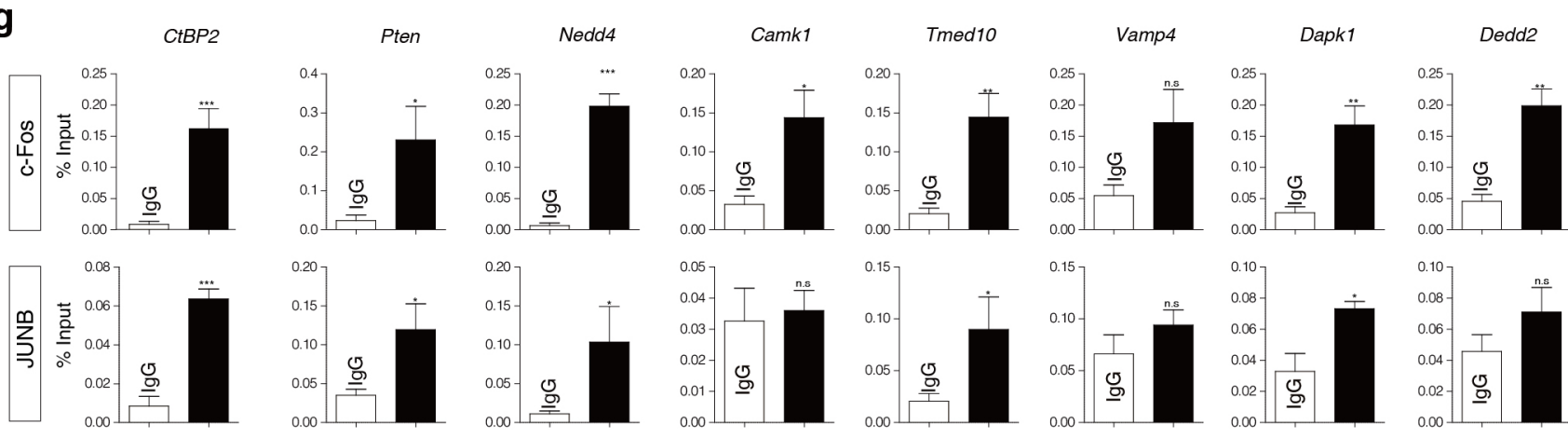

Extended Data Fig. 11 The AP-1 transcription factors are associated with KDM3B in mice retinas. a, The sequence logos representation of the 2 motifs identified using MEME-ChIP. De novo motif analysis was performed using sequences within a $\pm 150 \mathrm{bp}$ window of KDM3B ChIP-Seq peaks. The sequence logo size indicates nucleotide frequency and the P-values represent the significance of motif enrichments compared to that of the genomic background. b. Whole retinal lysates of 2-, 6-, and 12-month-old mice were immunoblotted using anti-c-Fos and JUNB antibodies. $\beta$-actin was used for internal loading control. c, The expression level of Kdm3b, c-Fos, JUN, and JUNB transcripts in whole retina plotted for four developmental time points (1.5-, 3-, 6-, and 12-month-old). d, The expression level of Kdm3b, c-Fos, JUN, and JUNB transcripts in $K d m 3 b^{+/+}$and $K d m 3 b^{+/-}$mice retinas. e, The expression level of c-Fos, JUN, and JUNB transcripts in rod cell and s-cone-like cells ( $\left.\mathrm{rrlp}_{\mathrm{GFP}} \mathrm{Nrl}^{-/}\right)$plotted for three developmental time points (P10, P14, and P28) using public RNA-sequencing data (GSE 74660). f, Immunoblot showing co-immunoprecipitation of endogenous KDM3B with c-Fos and JUNB in human lung cancer cells. g, Recruitment and presence of $\mathrm{c}-\mathrm{Fos}$ and JUNB at indicated gene promoters were validated by ChIP-qPCR. Error bars show mean \pm SEM. $P$ values obtained by Student's t-test. $* * * P<0.001, * * P<0.01$, n.s, not significant. 


\section{Supplementary Data Fig. 1}

a
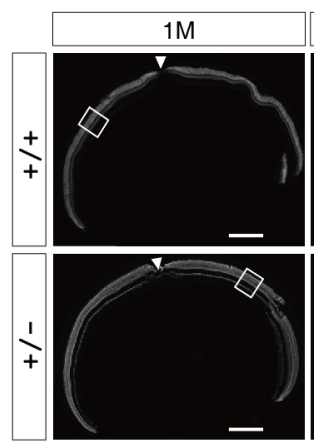

C

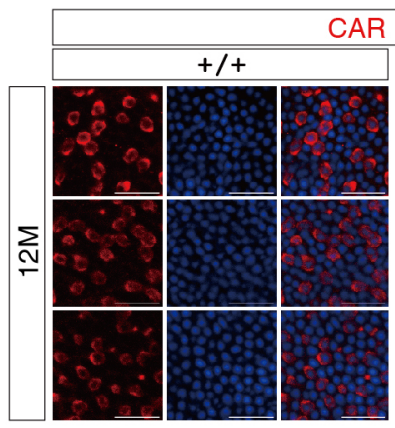

$6 \mathrm{M}$
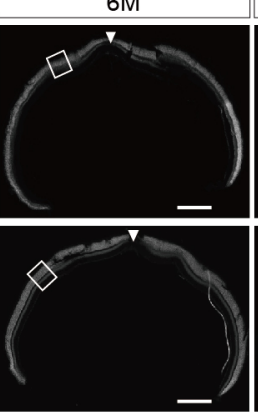

$+$

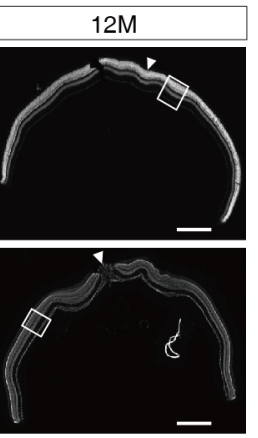

b

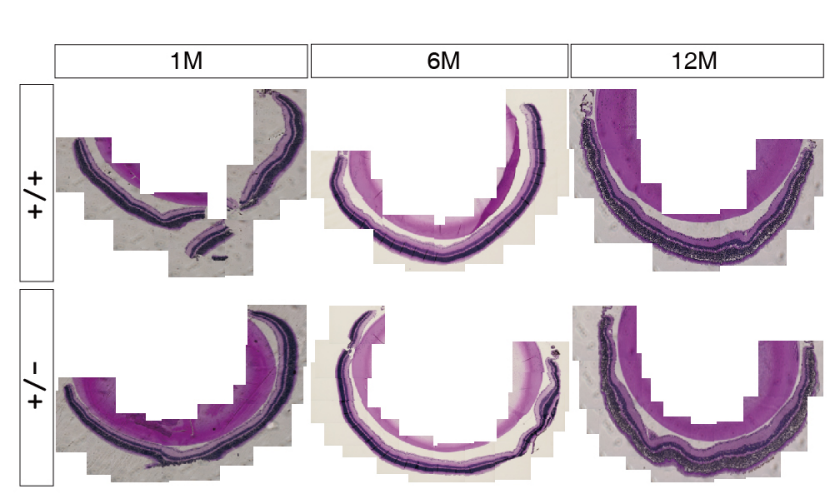

d

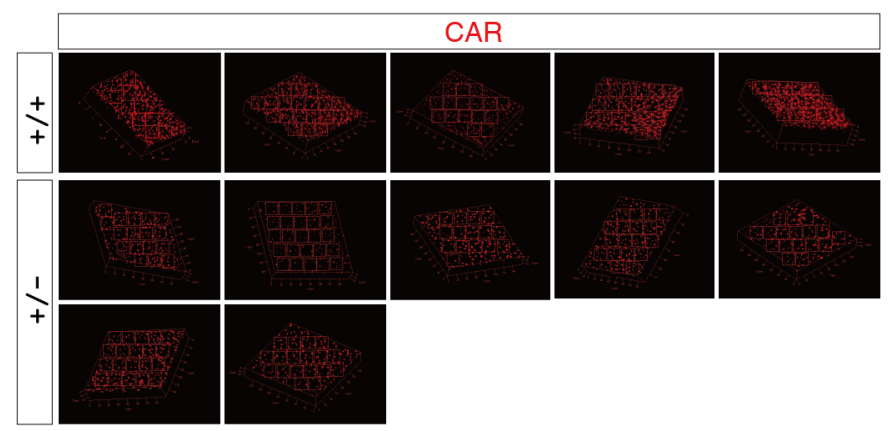

e

CAR DAPI

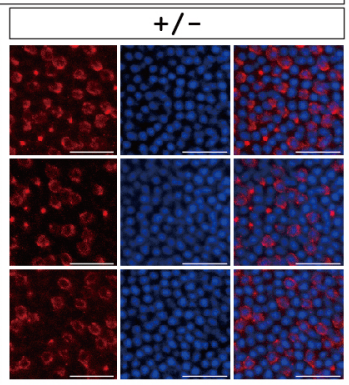

CAR
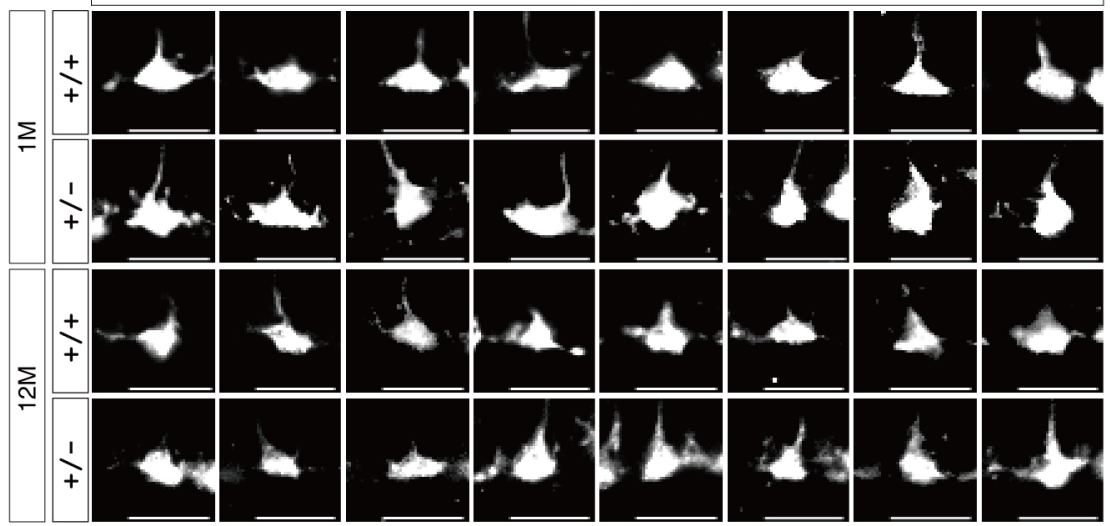

Supplementary Fig. 1 The morphological changes of rods and cones in $K d m 3 b^{+/+}$and $K d m 3 b^{+/}$mouse retina. a, Cross-sectioned retinas were stained with DAPI from 1-, 6-, and 12-month-old $K d m 3 b^{+/+}$and $K d m 3 b^{+/-}$mice. Scale bar: $500 \mu \mathrm{m}$. b, Representative images of hematoxylin and eosin (H\&E) staining for retinal thickness and total number of nuclei in ONL, INL, and GCL in the retinas of 1-, 6-, and 12-month-old $K d m 3 b^{+/+}$and $K d m 3 b^{+/}$mice. c,d, Immunostaining with CAR antibodies (red) of whole mount retina and DAPI (blue) in 12-month-old $K d m 3 b^{+/+}$and $K d m 3 b^{+/}$mice. Scale bar: $20 \mu \mathrm{m} \mathbf{e}$, The magnification of the cone pedicles in 1- and 12-months-old $K d m 3 b^{+/+}$and $K d m 3 b^{+/}$mice. Scale bar: $10 \mu \mathrm{m}$. 


\section{Supplementary Data Fig. 2}

a
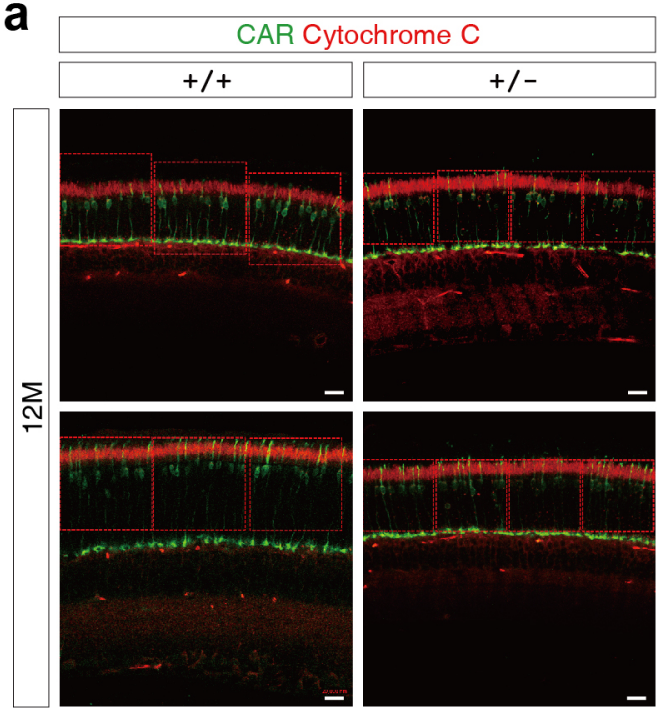

b
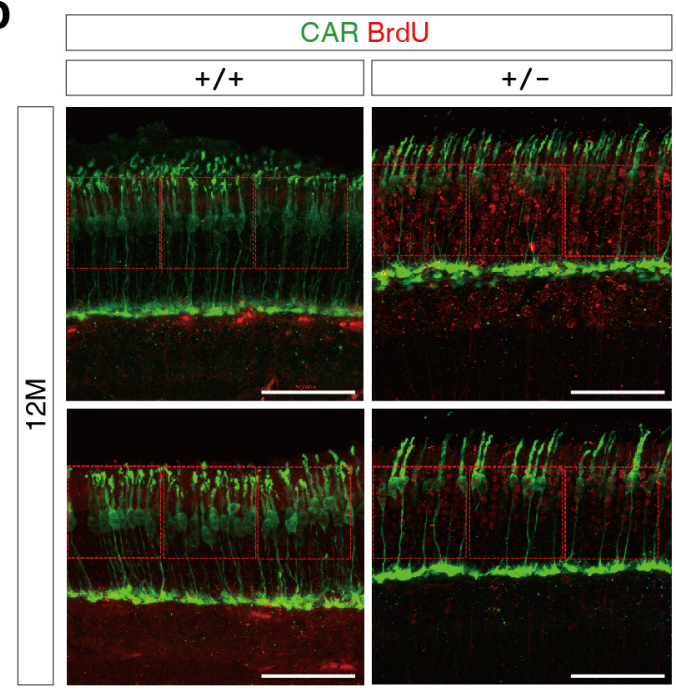

Supplementary Fig. 2 Induced apoptotic response of cone photoreceptors in $K d m 3 b^{+/}$retinas. a, Immunostaining of cytochrome c (red) and CAR (green) in 12-month-old $K d m 3 b^{+/+}$and $K d m 3 b^{+/-}$retinas. Scale bar: $20 \mu \mathrm{m}$. Cytochrome c-intensity is measured in red dashed box. b, Apoptotic cells were detected by TUNEL assay (BrdU positive cells) in 12-month-old $K d m 3 b^{+/+}$ and $K d m 3 b^{+/-}$mouse retinas. Scale bar: $50 \mu \mathrm{m}$. BrdU-positive cones in red dashed box are quantified. 


\section{Supplementary Data Fig. 3}

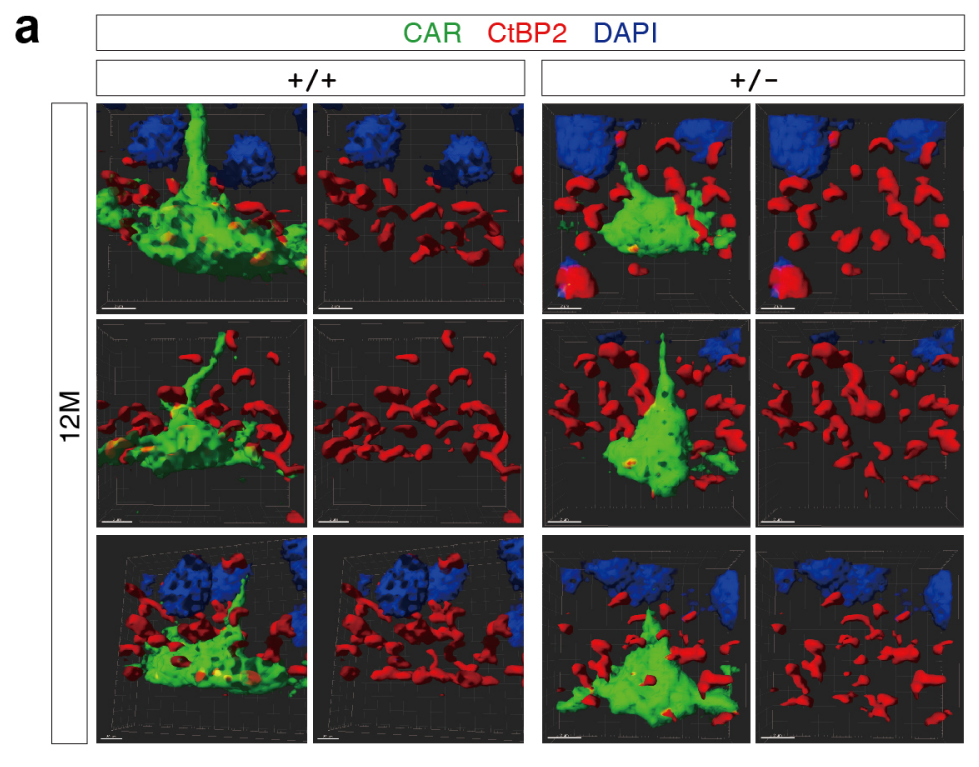

b

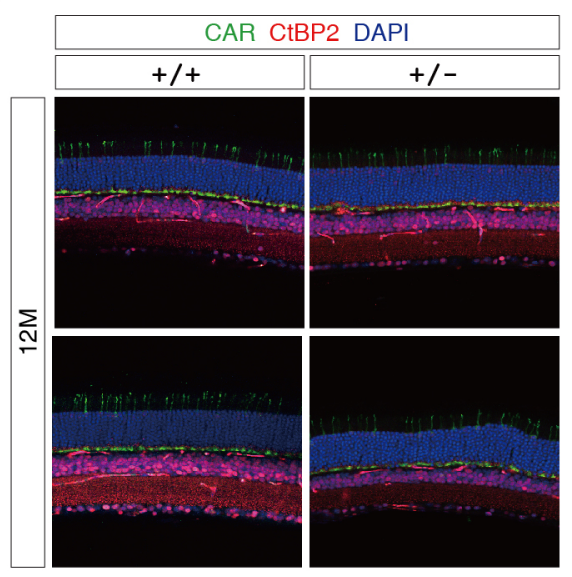

C
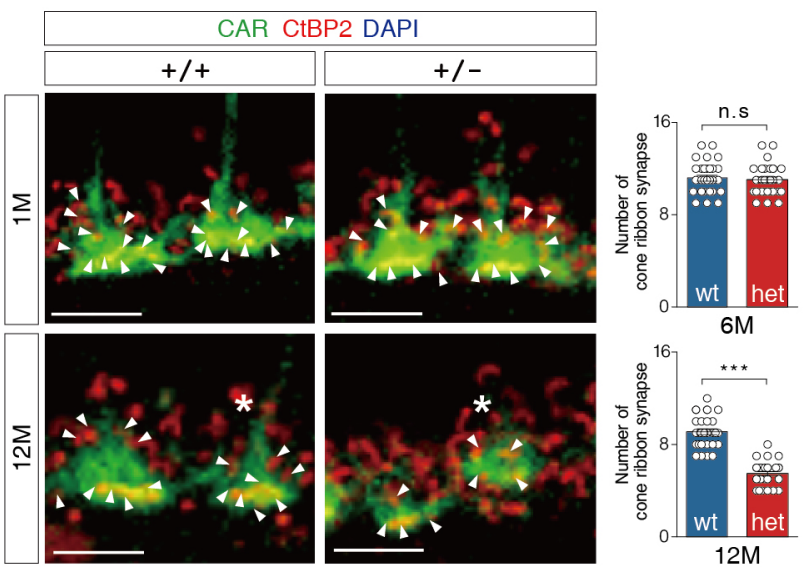

Supplementary Fig. 3 The alteration of synaptic morphology of cone photoreceptors in $K d m 3 b^{+/}$mice retina. a, Immunostaining of Cone arrestin (CAR) and CtBP2 in 12-month-old $K d m 3 b^{++/}$and $K d m 3 b^{+/}$mouse retina sections. Scale bar: $2 \mu \mathrm{m}$. b, The number of ribbon synapses in cone photoreceptor is quantified in 1- and 12-month-old $K d m 3 b^{+/+}$and $K d m 3 b^{+/}$mouse retinas (n $\left.=30\right)$ Scale bar: $20 \mu \mathrm{m}$. c, 3D reconstruction images showing ribbon synapses in cone photoreceptors of 12-month-old $K d m 3 b^{+/+}$and $K d m 3 b^{+/}$mouse retinas. Scale bar: $5 \mu \mathrm{m}$ 


\section{Supplementary Files}

This is a list of supplementary files associated with this preprint. Click to download.

- Supplementaryvideo1MP4.mp4

- Supplementaryinformation220103.docx

- SupplementaryTables.xlsx

- Supplementaryvideo2MP4.mp4 